\title{
Multi-Mechanism Flood Hazard Assessment: Example Use Case Studies
}

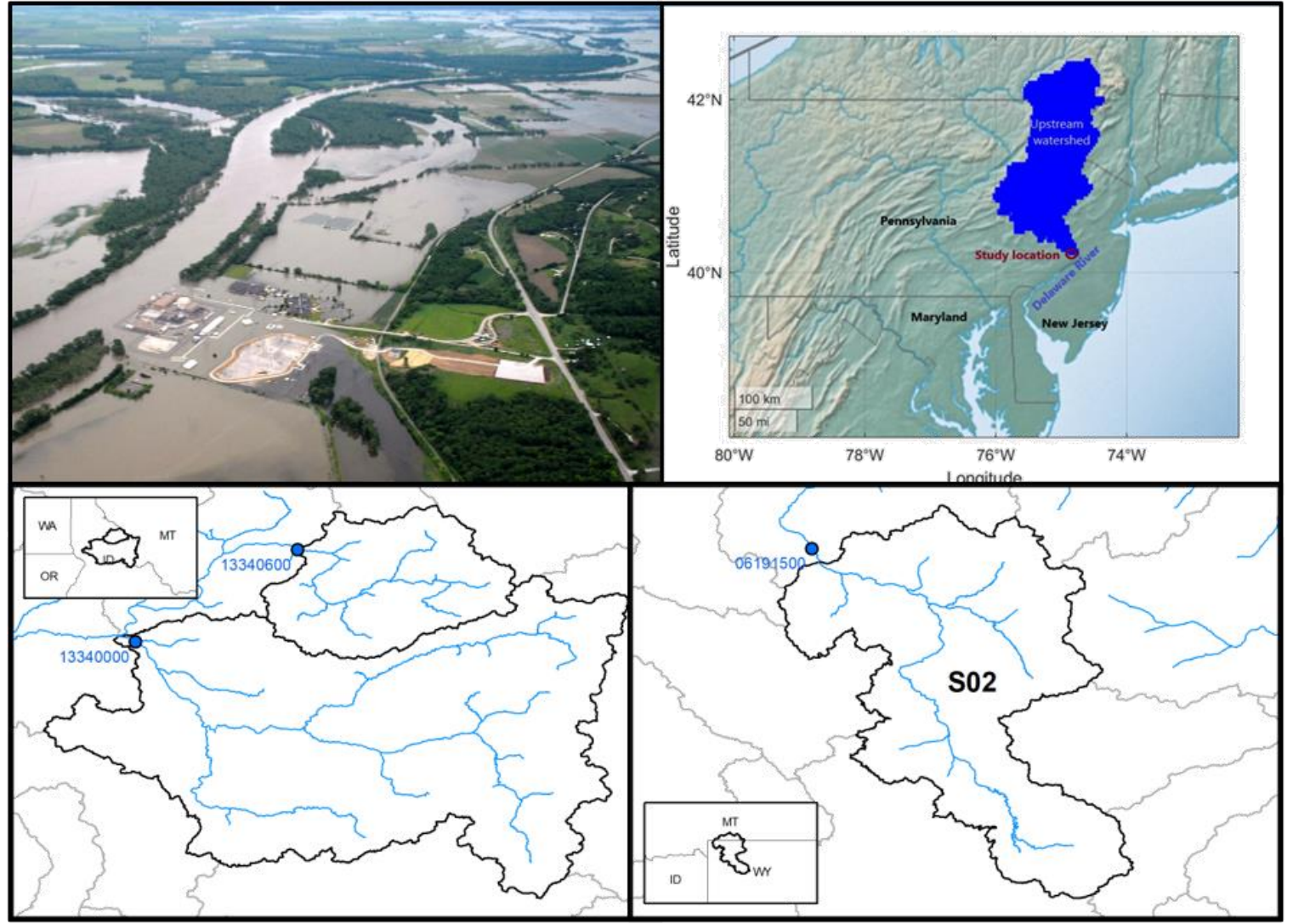

Somayeh Mohammadi

Michelle Bensi

Shih-Chieh Kao

Scott T. DeNeale

Approved for public release.

Distribution is unlimited.

October 2021 


\title{
DOCUMENT AVAILABILITY
}

Reports produced after January 1, 1996, are generally available free via US Department of Energy (DOE) SciTech Connect.

Website www.osti.gov

Reports produced before January 1, 1996, may be purchased by members of the public from the following source:

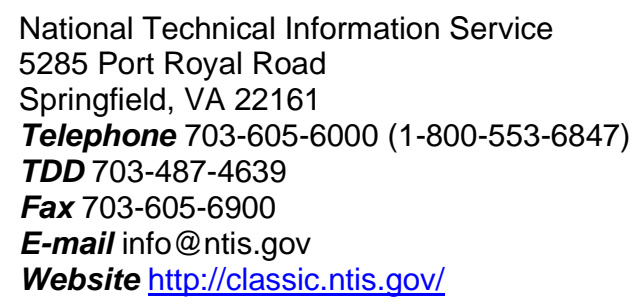

Reports are available to DOE employees, DOE contractors, Energy Technology Data Exchange representatives, and International Nuclear Information System representatives from the following source:

Office of Scientific and Technical Information

PO Box 62

Oak Ridge, TN 37831

Telephone 865-576-8401

Fax 865-576-5728

E-mail reports@osti.gov

Website https://www.osti.gov/

\begin{abstract}
This report was prepared as an account of work sponsored by an agency of the United States Government. Neither the United States Government nor any agency thereof, nor any of their employees, makes any warranty, express or implied, or assumes any legal liability or responsibility for the accuracy, completeness, or usefulness of any information, apparatus, product, or process disclosed, or represents that its use would not infringe privately owned rights. Reference herein to any specific commercial product, process, or service by trade name, trademark, manufacturer, or otherwise, does not necessarily constitute or imply its endorsement, recommendation, or favoring by the United States Government or any agency thereof. The views and opinions of authors expressed herein do not necessarily state or reflect those of the United States Government or any agency thereof.
\end{abstract}

Cover credits: Photograph (top-left) of Missouri River flooding at Fort Calhoun Nuclear Power Plant reprinted from Unknown Author and made available under a Creative Commons Attribution 3.0 Unported license. 
Environmental Sciences Division

\title{
MULTI-MECHANISM FLOOD HAZARD ASSESSMENT: EXAMPLE USE CASE STUDIES
}

\author{
Somayeh Mohammadi* \\ Michelle Bensi* \\ Shih-Chieh Kao ${ }^{\dagger}$ \\ Scott T. DeNeale $\dagger$
}

*University of Maryland, College Park

$\dagger$ Oak Ridge National Laboratory

October 2021

Prepared for

US Nuclear Regulatory Commission

Rockville, MD

\author{
Prepared by \\ OAK RIDGE NATIONAL LABORATORY \\ Oak Ridge, TN 37831-6283 \\ managed by \\ UT-BATTELLE, LLC \\ for the \\ US DEPARTMENT OF ENERGY \\ under contract DE-AC05-00OR22725
}





\section{CONTENTS}

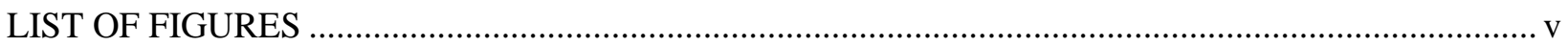

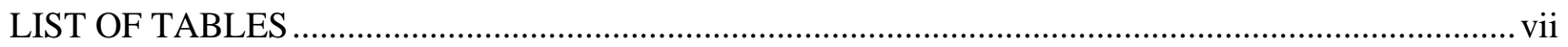

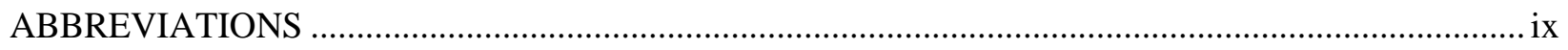

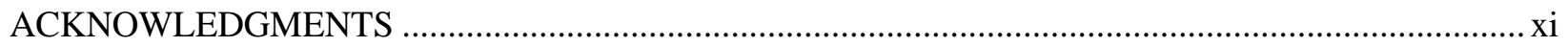

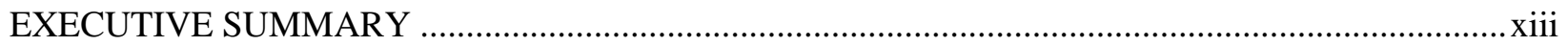

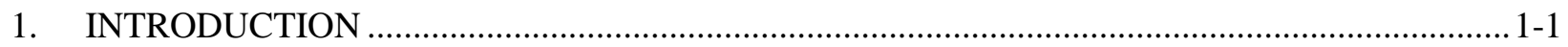

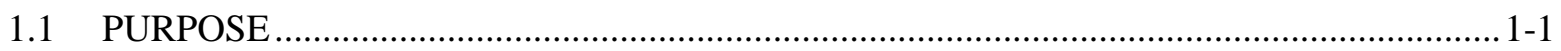

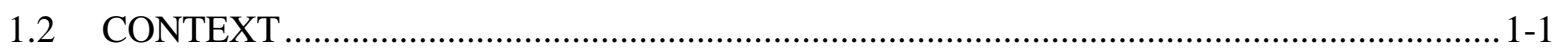

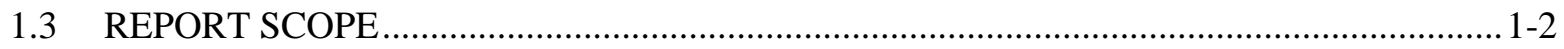

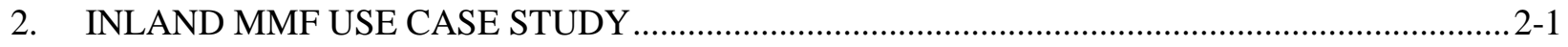

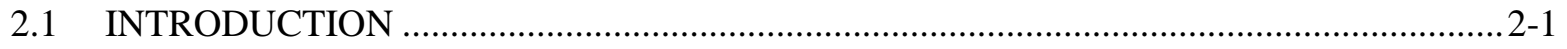

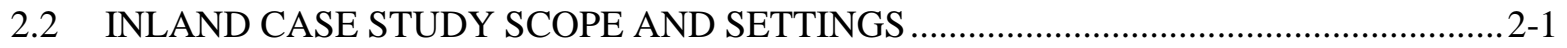

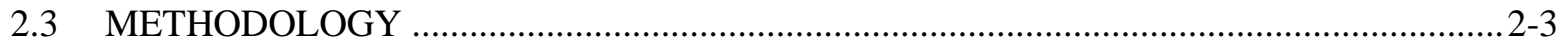

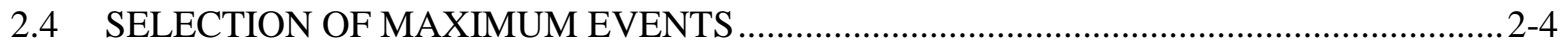

2.5 FITTING AND SELECTION OF MARGINAL DISTRIBUTIONS .............................. 2-5

2.5.1 Comparison across different maximum events .......................................................... 2-6

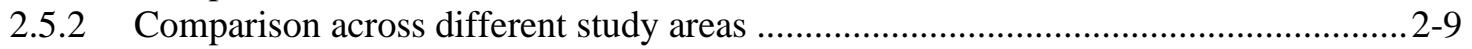

2.6 FITTING AND SELECTION OF COPULA FUNCTIONS …............................................. $2-12$

2.7 CONSTRUCTION OF JOINT DISTRIBUTIONS AND POTENTIAL

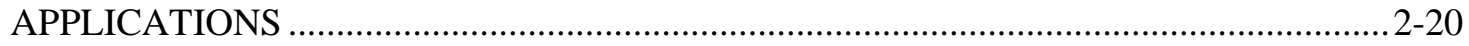

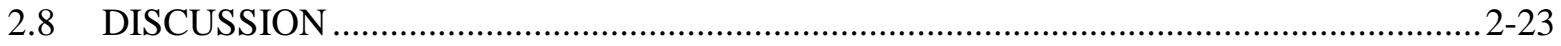

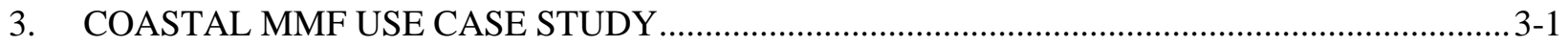

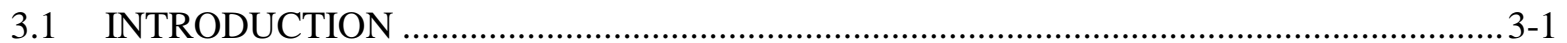

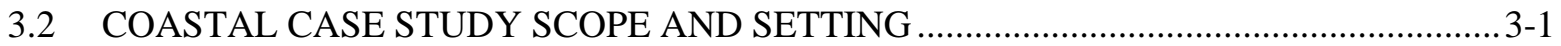

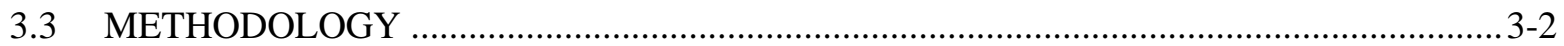

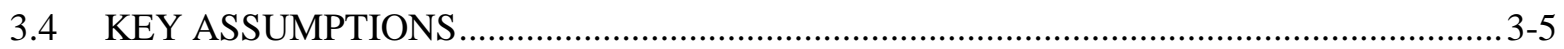

3.4.1 Storm parameters ........................................................................................

3.4.2 Storm surge model ................................................................................... $3-7$

3.4.3 Wind velocity model................................................................... $3-13$

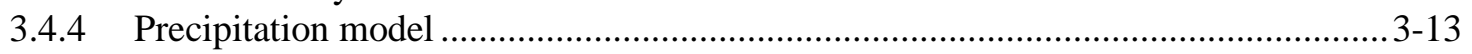

3.4.5 Precipitation-induced discharge model ........................................................... 3-18

3.4.6 Surge-, tide-, and river base flow-induced discharge model (combined

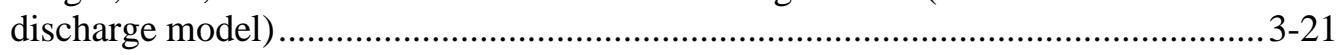

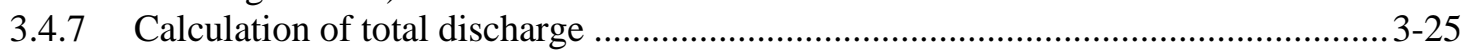

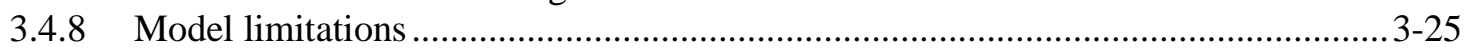

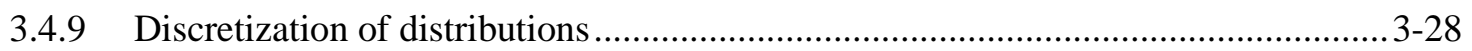

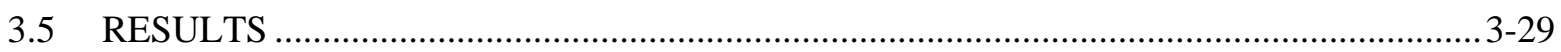

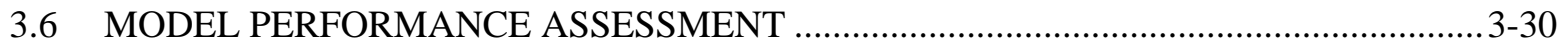

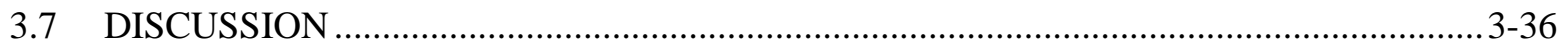

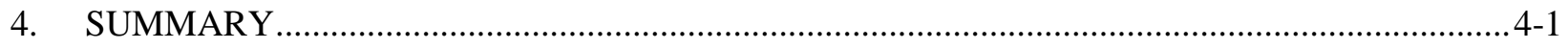

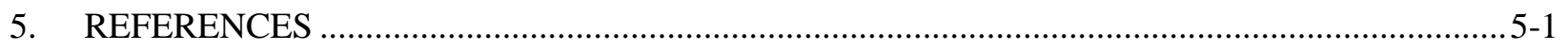





\section{LIST OF FIGURES}

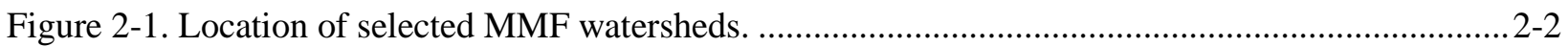

Figure 2-2. Assessment procedures of inland copula-based analysis............................................... 2-4

Figure 2-3. Annual maximum timing (count) of the selected variables (S2; 1980-2015).....................2-4

Figure 2-4. An example of maximum event selection (left: M1; middle: M2; right: M3) for S2............2-5

Figure 2-5. Fitting of $Q_{d y}$ using maximum events identified by (left) M1, (middle) M2, and (right)

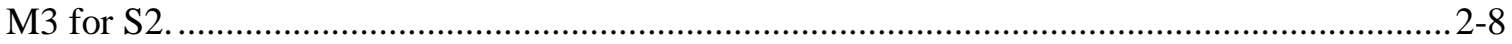

Figure 2-6. Fitting of $P_{3 d}$ using maximum events identified by (left) M1, (middle) M2, and (right)

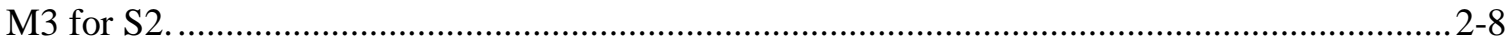

Figure 2-7. Fitting of $T_{3 d}$ using maximum events identified by (left) M1, (middle) M2, and (right)

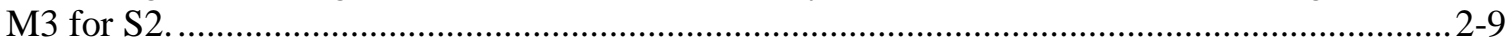

Figure 2-8. Fitting of $d S_{3 d}$ using maximum events identified by (left) M1, (middle) M2, and

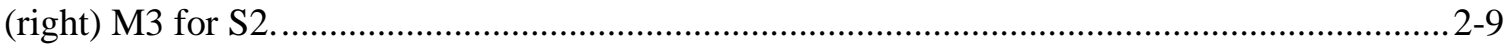

Figure 2-9. Fitting of $Q_{d y}$ using M2 maximum events for (left) S1, (middle) S2, and (right) S3..........2-10

Figure 2-10. Fitting of $P_{3 d}$ using M2 maximum events for (left) S1, (middle) S2, and (right) S3 .........2-11

Figure 2-11. Fitting of $T_{3 d}$ using M2 maximum events for (left) $\mathrm{S} 1$, (middle) S2, and (right) S3..........2-11

Figure 2-12. Fitting of $d S_{3 d}$ using M2 maximum events for (left) S1, (middle) S2, and (right) S3........2-11

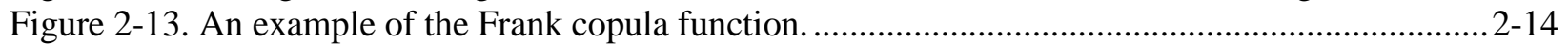

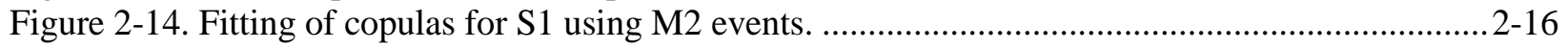

Figure 2-15. Fitting of copulas for S2 using M2 events. .............................................................. 2-17

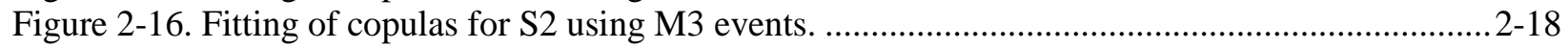

Figure 2-17. Fitting of copulas for S3 using M2 events. ................................................................ 2-19

Figure 2-18. Example of constructed bivariate joint distributions by copulas for S2 using M2

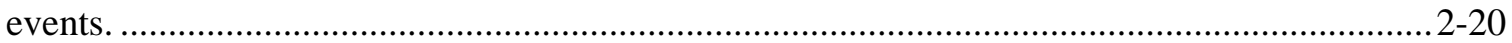

Figure 2-19. Joint distributions and conditional hazard curves of S1 using M2 events.........................2-21

Figure 2-20. Joint distributions and conditional hazard curves of S2 using M2 events........................2-21

Figure 2-21. Joint distributions and conditional hazard curves of S2 using M3 events.........................2-22

Figure 2-22. Joint distributions and conditional hazard curves of S3 using M2 events........................ 2-22

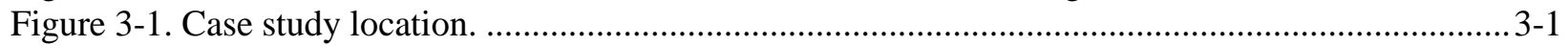

Figure 3-2. Location of the USGS gage, NOAA tide gage, save point 5373, and save point 7624......... 3-2

Figure 3-3. BN representation of fundamental integral used in case study. ......................................... 3-4

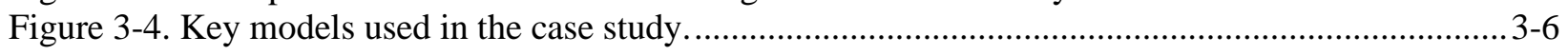

Figure 3-5. Key models used to develop BN representation used in the case study.............................. 3-6

Figure 3-6. Correlation between ADCIRC-simulated surge height and predicted surge height using GPR, NNET, and SVM models................................................................................

Figure 3-7. NACCS ADCIRC-computed storm surge at the target save point for 1,031 storms..............3-9

Figure 3-8. (left) Scatterplot of surrogate and numerical (ADCIRC) model predictions related to

surge height $(\mathrm{m})$ for the out-of-sample predictions for the 50 holdout sets, and (right)

histogram of computed correlation coefficients across the 50 holdout sets.......

Figure 3-9. (left) Scatterplot of computed out-of-sample prediction error and numerical

(ADCIRC) model predictions for across the 50 holdout sets, and (right) histogram of

computed RMSE values across the 50 holdout sets............................................................. $3-11$

Figure 3-10. Surrogate model-predicted partial-variable response functions. .................................... 3-12

Figure 3-11. Statistical wind velocity prediction model used in this study and plotted

North American Hurricane Database (HURDAT2) data........................................................ 3-13

Figure 3-12. Decay of rainfall rate with radial distance using a TRR model (Tuleya et al. 2007)..........3-15

Figure 3-13. Gridded points in the watershed upstream of case study location.....................................3-16

Figure 3-14. Examples of hurricane track wind decay; color key represents wind speeds $(\mathrm{km} / \mathrm{h}) \ldots \ldots \ldots . .3-17$ 
Figure 3-15. Hurricane-induced precipitation in the upstream area for an example storm track (red) at discrete time steps after storm landfall; the red star represents storm center at specific points after landfall; the color key represents rainfall (in./h).

Figure 3-16. Total daily rainfall for the upstream watershed for an example storm track (red); the color key represents rainfall (in./day).

Figure 3-17. Time series of (top) runoff and (bottom) basin-wide average daily precipitation............... 3-19

Figure 3-18. Scatterplot of runoff and basin-wide average daily precipitation.

Figure 3-19. Scatterplot of runoff and basin-wide average daily precipitation superimposed with linear and polynomial regression lines

Figure 3-20. (left) Scatterplot of surrogate and numerical (RAPID-VIC) model predictions related to discharge (cfs) for the out-of-sample predictions for the 50 holdout sets, and (right) histogram of computed correlation coefficients across the 50 holdout sets....

Figure 3-21. (left) Scatterplot of computed out-of-sample prediction error and numerical (RAPIDVIC) model predictions for across the 50 holdout sets, and (right) histogram of computed RMSE values across the 50 holdout sets.

Figure 3-22. (left) Time series of stage and discharge and (right) a scatterplot of surge and discharge.

Figure 3-23. Scatterplot of stage and discharge with fitted polynomial models of varying degrees.......3-22

Figure 3-24. (left) Scatterplot of recorded stage and discharge and (right) the recorded time series; poor-fitting points are highlighted in red.

Figure 3-25. Boxplots of (left) RMSE and (right) the correlation coefficient $(R)$ between predicted and observed values for holdout validation.

Figure 3-26. (left) Normalized frequency histogram of the selected data set along with PDFs fitted for candidate distributions, and (right) empirical CDF for the selected data set along with CDFs fitted for candidate distributions.

Figure 3-27. (left) Normalized frequency histogram of the selected data set along with LN PDF, and (right) empirical CDF for the selected data set along with LN CDF.

Figure 3-28. Empirical (left) CDF and (right) PMF related to high and low tides............................. 3-25

Figure 3-29. The location of USGS gage, rock riffles and save point 5373 .....................................3-26

Figure 3-30. Location of save points 5373 and 7624 and the NOAA tide gage................................. 3-27

Figure 3-31. ADCIRC-simulated surge height $(\mathrm{m})$ for save points 7624 and 5373............................ 3-27

Figure 3-32. Illustration of Monte Carlo simulation approach for generating CPTs............................3-29

Figure 3-33. Total discharge hazard curve (presented for illustrative purposes).................................3-30

Figure 3-34. Synthetic and observed storm track related to Hurricane Isaias........................................3-32

Figure 3-35. Synthetic and observed storm track related to Tropical Storm Fay. ..................................3-33

Figure 3-36. Synthetic and observed storm track related to Hurricane Irene. .......................................3-33

Figure 3-37. Location of the representative point for extracting information at landfall

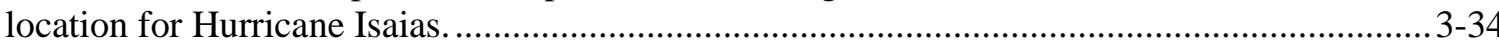

Figure 3-38. Location of the representative point for extracting information at landfall location for Tropical Storm Fay.................................................................................................. 3-34

Figure 3-39. Location of the representative point for extracting information at landfall location

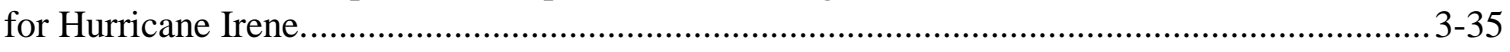

Figure 3-40. Posterior distribution of the modeled discharge values for Hurricane Isaias..................... 3-35

Figure 3-41. Posterior distribution of the modeled discharge values for Tropical Storm Fay.................3-36

Figure 3-42. Posterior distribution of the modeled discharge values for Hurricane Irene......................3-36 


\section{LIST OF TABLES}

Table 2-1. Selected watersheds in the inland MMF case study. ....

Table 2-2. Fitting of marginal distributions using three maximum event searching approaches for

S2 (USGS ID: 06191500).

Table 2-3. Fitting of marginal distributions using M2 for the three study areas..................................2-10

Table 2-4. Selected marginal distribution and fitted parameters. .....................................................2-12

Table 2-5. Fitting of copula function for the four selected cases........................................................2-15

Table 2-6. Selected copula functions and fitted parameters. .............................................................2-20

Table 3-1. Distributions and corresponding parameters for hurricane parameters..................................3-7

Table 3-2. Correlation and RMSE values related to GPR, NNET, and SVM models............................. 3-9

Table 3-3. Bias-corrected constants for the TRR model..................................................................... 3-14

Table 3-4. Wind decay model coefficients for the area under study.................................................. 3-16

Table 3-5. AIC and BIC values estimated for candidate distributions for base flow............................3-24

Table 3-6. Discretized values for the parameters required in the Bayesian formulation. ........................3-28

Table 3-7. Storm parameters and surge and discharge values related to USGS gage and modeling results. 



\section{ABBREVIATIONS}

\begin{tabular}{|c|c|}
\hline ADCIRC & Advanced Circulation \\
\hline AIC & Akaike information criterion \\
\hline BIC & Bayesian information criterion \\
\hline $\mathrm{BN}$ & Bayesian network \\
\hline CAMELS & Catchment Attributes and Meteorological for Large-sample Studies \\
\hline $\mathrm{CDF}$ & cumulative distribution function \\
\hline CLT & Clayton \\
\hline $\mathrm{CM}$ & Cramér-von Mises \\
\hline CPT & conditional probability table \\
\hline FRK & Frank \\
\hline GAU & Gaussian \\
\hline GEV & generalized extreme value \\
\hline GM & gamma \\
\hline GPR & Gaussian process regression \\
\hline GUM & Gumbel \\
\hline JCDF & joint-cumulative distribution function \\
\hline KS & Kolmogorov-Smirnov \\
\hline LN & lognormal \\
\hline LP3 & log-Pearson type III \\
\hline M1 & univariate maximum \\
\hline M2 & multivariate peak-over-threshold \\
\hline M3 & maximum joint empirical probability \\
\hline MMF & multi-mechanism flood \\
\hline NACCS & North Atlantic Comprehensive Coastal Study \\
\hline NHDPlus & National Hydrography Dataset Plus \\
\hline NNET & neural network \\
\hline NOAA & National Oceanic and Atmospheric Administration \\
\hline NOR & normal \\
\hline NRC & US Nuclear Regulatory Commission \\
\hline PDF & probability density function \\
\hline PFHA & probabilistic flood hazard assessment \\
\hline PMF & probability mass function \\
\hline RAPID & Routing Application for Parallel Computation of Discharge \\
\hline RMSE & root mean square error \\
\hline S1 & Clearwater River at Orofino, Idaho \\
\hline $\mathrm{S} 2$ & Yellowstone River at Corwin Springs, Montana \\
\hline S3 & NF (North Fork) Clearwater River NR (near) Canyon Ranger Station, Idaho \\
\hline SVM & support vector machine \\
\hline SWE & snow water equivalent \\
\hline TD2 & $\mathrm{t}$ with degree of freedom $=2$ \\
\hline TRR & Tropical Rainfall Measuring Mission rain rates \\
\hline USACE & US Army Corps of Engineers \\
\hline VIC & Variable Infiltration Capacity \\
\hline
\end{tabular}





\section{ACKNOWLEDGMENTS}

\section{US Nuclear Regulatory Commission}

The authors would like to acknowledge and express their appreciation to the US Nuclear Regulatory Commission (NRC) for overseeing and funding this research. The following NRC staff were heavily involved in supporting this research effort:

- Elena Yegorova

- Meredith Carr

- Joseph Kanney

- Yuan Cheng

\section{Oak Ridge National Laboratory}

The authors would like to thank the following individuals from Oak Ridge National Laboratory (ORNL) for providing technical review and support for this report and associated review:

- Sudershan Gangrade

- Olivia Shafer

The discussion of specific references, methods, software, or tools in this report does not constitute endorsement or approval for any specific use by ORNL, the University of Maryland, or NRC. 



\section{EXECUTIVE SUMMARY}

Multi-mechanism flood (MMF) events are caused by the combined effects of more than one flooding mechanism. Although floods can result from the occurrence of individual flood mechanisms, they can (and often do) result from multiple flooding mechanisms. MMF events may be more severe than singlemechanism events, or they may differ in characteristics. To facilitate comprehensive risk-informed decision-making to protect against and mitigate the effects of flood events, understanding the hazard contributions from MMFs is important. Nevertheless, conventional probabilistic flood hazard assessment approaches typically focus on individual flood hazard mechanisms. This report is part of a research project funded by the US Nuclear Regulatory Commission (NRC) intended to assist NRC in developing the technical basis for guidance on developing probabilistic estimates of flood hazards for combinations of flood mechanisms. Specifically, the purpose of this report is to document two case studies to illustrate approaches for quantifying MMF hazards for inland and coastal flooding scenarios.

This report documents the third of a series of tasks under the NRC-sponsored research project Methods for Estimating Joint Probabilities of Coincident and Correlated Flooding Mechanisms for Nuclear Power Plant Flood Hazard Assessments. These three main project research tasks are as follows:

- $\quad$ Task 1: Survey of current concepts and methods in assessing MMF hazards

- $\quad$ Task 2: Critical assessment of selected methods and approaches for quantifying probabilistic MMF hazard risk

- $\quad$ Task 3: Development of example case studies to illustrate best practices for quantifying probabilistic MMF hazard risk

Tasks 1 and 2 have been published by Bensi et al. (2020), which summarized the current state of practice in the probabilistic assessment of MMFs. Based on the critical review and insights developed under Tasks 1 and 2, the research team selected two case studies to illustrate approaches for quantifying probabilistic MMF hazards. These example case studies leverage empirical data, as well as synthetic data generated from numerical and surrogate modeling approaches. The case studies use Bayesian-motivated and copula-based approaches and were developed for inland and coastal flooding hazards. Specifically, the first case study focuses on rainfall- and snowmelt-driven extreme streamflow events. It uses copulas as the main computational approach. The second case study considers coastal flooding hazards caused by rainfall and storm surge and uses a Bayesian-motivated approach for probabilistic hazard assessment. 



\section{INTRODUCTION}

\subsection{PURPOSE}

Multi-mechanism flood (MMF) events are caused by the combined effect of more than one flooding mechanism (e.g., flood events caused by the simultaneous occurrence of precipitation-induced river flooding and storm surge). Most conventional probabilistic flood hazard assessment (PFHA) approaches focus on individual flood hazard mechanisms. However, MMF events may be more severe than singlemechanism events, or they may differ in characteristics. Therefore, realistic probabilistic assessment of flooding hazards requires consideration of MMFs. The purpose of this report is to document two case studies to illustrate approaches for quantifying probabilistic MMF hazards.

\subsection{CONTEXT}

This report was developed through a research project funded by the US Nuclear Regulatory Commission (NRC) intended to assist NRC in developing the technical basis for guidance on developing probabilistic estimates of flood hazards for combinations of flood mechanisms. This research project is a part of the NRC PFHA Research Program and will support development of guidance on the use of PFHA methods in safety evaluations for existing or proposed US nuclear power plant infrastructure.

According to the NRC PFHA Research Plan (NRC 2014),

the current limited risk-informed guidance with respect to flooding constitutes a significant gap in the NRC's risk-informed, performance-based regulatory approach to the assessment of natural hazards and potential consequences for safety of commercial nuclear facilities.

According to Safety Strategy 2 of the NRC Strategic Plan for Fiscal Years 2018-2022 (NRC 2018), NRC aims to

further risk-inform the current regulatory framework in response to advances in science and technology, policy decisions, and other factors, including prioritizing efforts to focus on the most safety-significant issues.

To support this strategy, NRC (NRC 2018) identifies a contributing activity to

conduct research activities to confirm the safety of operations and enhance the regulatory framework by addressing changes in technology, science, and policies.

The research effort documented in this report supports this activity.

This report documents the third of a series of tasks under the NRC-sponsored research project Methods for Estimating Joint Probabilities of Coincident and Correlated Flooding Mechanisms for Nuclear Power Plant Flood Hazard Assessments. These three main project research tasks are as follows:

- $\quad$ Task 1: Survey of current concepts and methods in assessing MMF hazards

- Task 2: Critical assessment of selected methods and approaches for quantifying probabilistic MMF hazard risk

- Task 3: Development of example case studies to illustrate best practices for quantifying probabilistic MMF hazard risk 
Tasks 1 and 2 have been completed. The main outcomes of Tasks 1 and 2 include documentation of (1) a reconnaissance-level survey of the current state of concepts and practice for MMF hazard assessment; (2) a generalized MMF assessment framework to address the distinctions among flood-forcing phenomena, flood mechanisms (which are categorized into three types of mechanisms), and flood severity metrics; (3) a wide-ranging survey of approaches and methods that have been applied to various flooding phenomena and settings; and (4) a critical assessment of MMF hazard assessment methods. The critical review placed particular emphasis on assessing research and applied guidance from the perspective of nuclear power plant and other critical infrastructure applicability, which focuses on severe events with low annual frequencies of exceedance (long return periods). The methods and results of Tasks 1 and 2 are documented elsewhere (Bensi et al. 2020).

Based on the critical review and insights developed in performing Tasks 1 and 2, the research team selected two case studies to illustrate approaches for probabilistic assessment of MMF hazards. These example case studies leverage empirical data as well as synthetic data generated from numerical and surrogate modeling approaches. The case studies use Bayesian-motivated and copula-based approaches and have been developed for inland and coastal flooding hazards.

\subsection{REPORT SCOPE}

This report documents two use case studies. The first case study focuses on rainfall- and snowmelt-driven extreme streamflow events. It uses copulas as the main computational approach. The case study application is intended to demonstrate

(1) general procedures to construct multivariate joint distributions using copulas,

(2) the identification of extreme samples for multivariate frequency analysis,

(3) the selection of suitable marginal distributions and copula functions,

(4) applications of copula-derived joint distributions in PFHA, and

(5) strengths and limitations of the copula-based MMF assessment approach.

The second case study considers coastal hazards and uses a Bayesian-motivated approach for probabilistic hazard assessment. The case study builds off of the established joint probability method commonly employed for the assessment of tropical cyclone-induced coastal storm surge hazards (Toro et al. 2010). The flood forcing phenomenon is a tropical cyclone (hurricane), and the flood mechanisms involved in the analysis include hurricane-induced surge, precipitation, and river flow. The research objective is to develop a hazard curve for river discharge accounting for the effects of river base flow, hurricane-induced surge, tides, and precipitation-induced runoff. The case study application is intended to demonstrate

(1) the general conceptual approach to construct multivariate joint distributions using Bayesian modeling approaches,

(2) the development and use of requisite marginal and conditional distributions (including use of numerical, empirical, or surrogate models to define conditional relationships between quantities), and

(3) the quantification of joint distributions and development of hazard curves. 
In both cases, the studies demonstrate processes and concepts. They include the use of illustrative models and assumptions and do not provide a definitive hazard assessment for the target location. 


\section{INLAND MMF USE CASE STUDY}

\subsection{INTRODUCTION}

Among various potential hazards, combined rainfall- and snowmelt-driven extreme streamflow events were studied. With the trend of earlier snowmelt in recent decades (mostly due to atmospheric warming; Ashfaq et al. 2013), there is a growing interest in understanding how peak streamflow estimates and the corresponding hazard curves may be affected by the co-occurrence of major streamflow and snowmelt events. As opposed to the conventional univariate analysis that only analyzes the time series of streamflow to derive hazard curves, copulas were used to construct joint distributions that combine multiple variables (e.g., streamflow, precipitation, temperature, and snowmelt) for the derivation of conditional hazard curves. This inland case study serves as an example of copula-based analysis, which can be expanded for broader MMF analyses in a variety of PFHA applications. It should be interpreted as a demonstration of a process rather than a definitive hazard assessment for the target location.

\subsection{INLAND CASE STUDY SCOPE AND SETTINGS}

To select suitable study areas (watersheds) in this case study, the following criteria were considered:

- Long-term historic streamflow observations should be available at the watershed outlet to support model validation and frequency analysis.

- Existing hydrologic model with acceptable performance should be available at the selected watershed to simulate snow processes and further data synthetization.

- To avoid overcomplicating the use case study, the watershed should not be under strong flow regulation (e.g., presence of major dams). A headwater basin is preferred.

- Significant snowpack is expected in the watershed to enable the assessment of snowmelt-influenced events.

To help effectively identify suitable study areas, watersheds documented in the Catchment Attributes and Meteorological for Large-sample Studies (CAMELS) data set (Newman et al. 2014; Addor et al. 2017) were leveraged. CAMELS is a community data set that provides daily meteorologic and streamflow observations for 671 small- to medium-sized watersheds across the contiguous United States. Watersheds in this data set have minimal human interference and span diverse climatic and geographical conditions, making it suitable for this analysis. Among CAMELS watersheds with snow fraction (i.e., fraction of snow days) greater than $40 \%$, three watersheds that have the largest annual average streamflow were selected:

- S1: Clearwater River at Orofino, Idaho (USGS ID: 13340000)

- S2: Yellowstone River at Corwin Springs, Montana (USGS ID: 06191500)

- S3: NF (North Fork) Clearwater River NR (near) Canyon Ranger Station, Idaho (USGS ID: 13340600)

Further information of these three selected study areas is shown in Table 2-1 and Figure 2-1. The size of these watersheds ranges from 3,357 to $1,4263 \mathrm{~km}^{2}$, and the mean elevation ranges from 569 to $2,542 \mathrm{~m}$. At each watershed, two types of data are available: 
(1) Observations: Given the design of CAMELS, long-term USGS daily streamflow gage stations are available at the outlet of each watershed. In addition, daily meteorological observations (e.g., precipitation, temperature) from Daymet (Thornton et al. 1997) were collected to support joint probability analysis.

(2) Observation-driven hydrologic model outputs: To simulate snow processes in each watershed, an existing contiguous US Variable Infiltration Capacity (VIC) model implemented by Oubeidillah et al. (2014) and Naz et al. (2016) was leveraged. The historic Daymet meteorological forcings were used to drive VIC to estimate surface runoff, base flow, evapotranspiration, snow water equivalent (SWE), and other hydrologic variables. The total runoff (surface runoff + base flow) from VIC was then used to simulate daily streamflow by the Routing Application for Parallel Computation of Discharge (RAPID) (David et al. 2011) routing model along the NHDPlus (National Hydrography Dataset Plus) (McKay et al. 2012) river network. Satisfactory model performance $\left(\mathrm{R}^{2}>0.7\right)$ was identified by comparing the simulated VIC-RAPID with observed USGS daily streamflow at each watershed outlet.

Table 2-1. Selected watersheds in the inland MMF case study.

\begin{tabular}{llccccc}
\hline $\begin{array}{c}\text { Site } \\
\text { ID }\end{array}$ & \multicolumn{1}{c}{ Site name } & $\begin{array}{c}\text { Watershed } \\
\text { area }\left(\mathbf{k m}^{2}\right)\end{array}$ & $\begin{array}{c}\text { USGS gage/ } \\
\text { period }\end{array}$ & $\begin{array}{c}\text { Annual mean } \\
\text { flow }\left(\mathbf{m}^{\mathbf{3}} / \mathbf{s}\right)\end{array}$ & $\begin{array}{c}\text { Mean } \\
\text { elevation (m) }\end{array}$ & $\begin{array}{c}\text { VIC-RAPID } \\
\text { daily R }\end{array}$ \\
\hline S1 & $\begin{array}{l}\text { Clearwater River at } \\
\text { Orofino, Idaho }\end{array}$ & 14,263 & $\begin{array}{c}13340000 \\
(1965-\text { present })\end{array}$ & 247.2 & 1451 & 0.74 \\
\hline S2 & $\begin{array}{l}\text { Yellowstone River at } \\
\text { Corwin Springs, Montana }\end{array}$ & 6,775 & $\begin{array}{c}06191500 \\
(1911-\text { present })\end{array}$ & 88.6 & 2542 & 0.80 \\
\hline S3 & $\begin{array}{l}\text { NF Clearwater River NR } \\
\text { Canyon Ranger Station, } \\
\text { Idaho }\end{array}$ & 3,357 & $\begin{array}{c}13340600 \\
(1867-\text { present })\end{array}$ & 98.2 & 569 & 0.71 \\
\hline
\end{tabular}
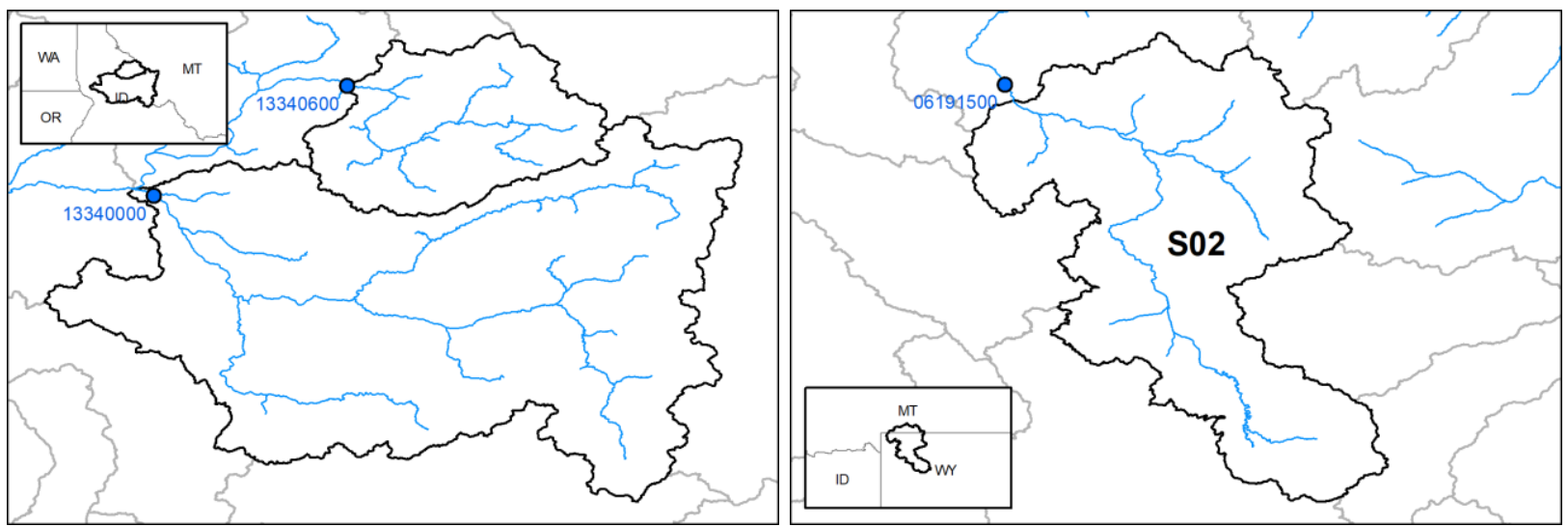

Figure 2-1. Location of selected MMF watersheds.

The case study specifically focused on four variables: daily streamflow $\left(Q_{d y}, \mathrm{~m}^{3} / \mathrm{s}\right), 3$-day total precipitation $\left(P_{3 d}, \mathrm{~mm}\right), 3$-day average temperature $\left(T_{3 d},{ }^{\circ} \mathrm{C}\right)$, and change of 3-day $\operatorname{SWE}\left(d S_{3 d}, \mathrm{~mm}\right)$. Daily streamflow $Q_{d y}$ has been the main target in a variety of flood hazard assessment studies. The other three variables were selected based on their potential relevance to snowmelt to explore how these variables may affect the development of MMF hazard curves. The timing of $Q_{d y}$ occurred at the third day of $P_{3 d}, T_{3 d}$, and $d S_{3 d}$. For convenience, the change of SWE was calculated by past minus future so that a positive value of SWE change refers to a reduction in SWE. 
Considering the common periods when all data are available, watershed-average daily time series of these four variables from 1980 to 2015 were calculated. Although gage-based snow observations are also available at some locations in the watersheds, they only reflect gage-specific snow cover conditions and cannot be used to represent the overall snowpack status in a watershed. The VIC hydrologic model, on the other hand, simulates snow processes within each grid cell with varying elevation bands across the entire watershed. Thus, VIC can be suitable to simulate the watershed-scale SWE status. The model-based SWE is a reasonable and sufficient choice to demonstrate multivariate copula analysis for the purpose of this case study. Alternative snow data can be explored when conducting site-specific MMF analysis at different study areas.

\subsection{METHODOLOGY}

The assessment of this inland copula-based MMF analysis includes the following steps. A conceptual diagram is illustrated in Figure 2-2.

(1) Selection of maximum events: Given that the maximum events of each variable occur at different time, the selection of maximum events in a multivariate setting becomes nontrivial. In this case study, different ways to define maximum events were tested, and their impacts on the constructed joint distributions were compared.

(2) Selection and fitting of marginal distributions: The conventional univariate frequency analysis approach was used to select and fit suitable marginal distribution of each variable. Commonly used probability density functions (PDFs) were tested and selected.

(3) Selection and fitting of copula functions: Conceptually similar to the selection of marginal distributions, several commonly used copula functions such as Frank, Gumbel, Clayton, Gaussian, and $t$ copulas were tested and selected to represent the dependence structures between pairs of variables.

(4) Goodness-of-fit tests: Rank-based empirical distribution and several commonly used goodness-of-fit tests (e.g., Kolmogorov-Smirnov [KS], Cramér-von Mises [CM], Akaike information criterion [AIC], Bayesian information criterion [BIC]) were used in the case study to demonstrate how these tests may be used to evaluate and select suitable marginal and copula functions.

(5) Construction of joint distributions: The forming of joint distribution through the connection of marginal distributions and a copula function was demonstrated. The challenges and some special considerations when constructing joint distributions with higher dimensions (i.e., greater than 2) are discussed.

(6) Application of derived joint distribution: Finally, how to use the derived joint distribution for potential PFHA applications is demonstrated (e.g., for given snow and temperature conditions, how to derive the conditional peak streamflow distribution and use them for flood hazard curve calculation). 


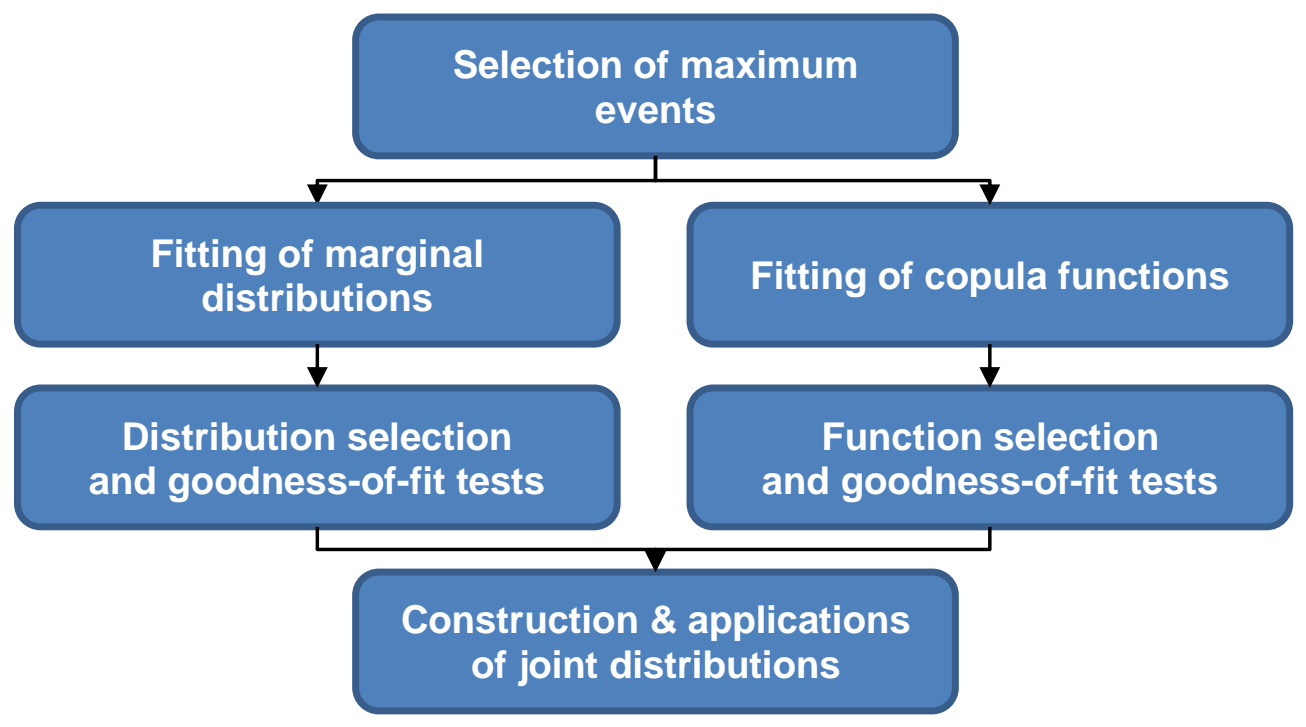

Figure 2-2. Assessment procedures of inland copula-based analysis.

\subsection{SELECTION OF MAXIMUM EVENTS}

In the conventional univariate frequency analysis, two methods are commonly used to select maximum events for PDF fitting: annual maximum, and peak-over-threshold (Rao and Hamed 2000). Both methods focus on identifying the maxima of a single variable without considering its relationships with other variables. Because the annual maximums of the variables occur at different times, selecting maximum events in a multivariate setting is challenging. Taking data from S2 as an example, the histograms of months when annual maximum events occur are shown in Figure 2-3. For this site, none of the variables had consistent annual maximum timing; most peaks for $d S, Q$, and $T$ occurred in April, May, and July, respectively, and $P$ had no clear peak. This suggests that different maximum event concepts and definitions are required for multivariate frequency analysis.
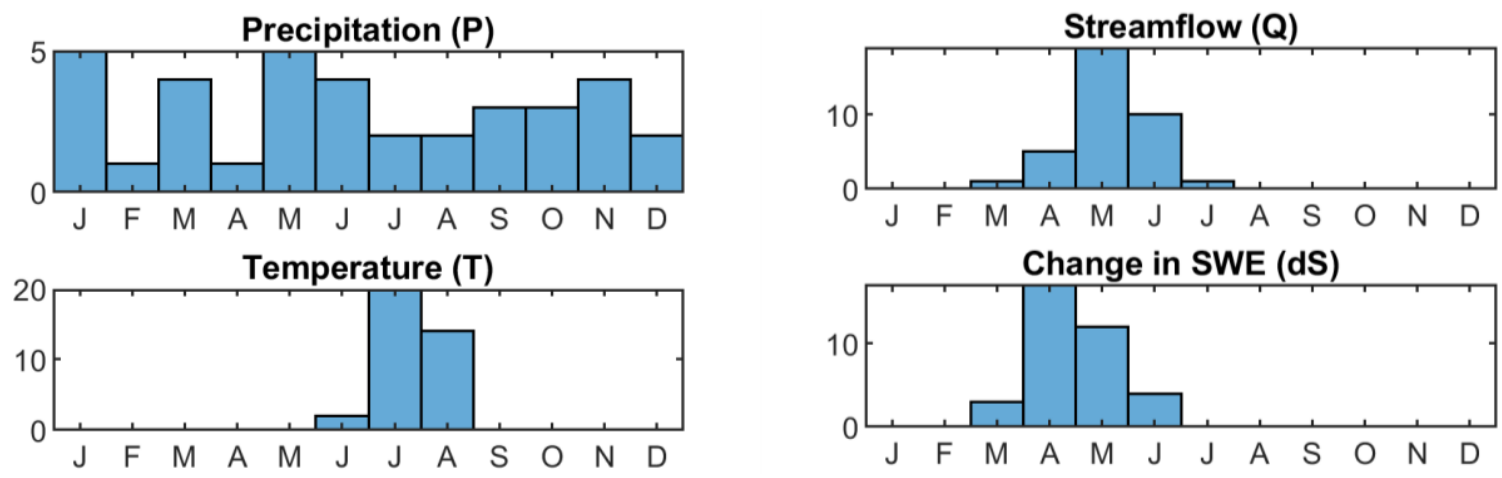

Figure 2-3. Annual maximum timing (count) of the selected variables (S2; 1980-2015).

To understand how different maximum event definitions may affect the constructed joint distributions, three ways to search maximum events were compared for each study area:

- M1-univariate maximum: In this approach, the maximum daily streamflow $\left(Q_{d y}\right)$ in each year was identified. The corresponding values of other variables $\left(P_{3 d}, T_{3 d}\right.$, and $\left.d S_{3 d}\right)$ were then identified to form the set of maximum events for fitting. Because the selection is only based on $Q_{d y}$, other variables can be small. 
- M2-multivariate peak-over-threshold: In this approach, an average sampling rate $r$ (e.g., 5 events per year) was set up. Then, a quantile threshold $q$ was gradually lowered from $100 \%$ to select events that satisfy $\left\{Q_{d y}>Q_{d y, q}\right\} \cap\left\{P_{3 d}>P_{3 d, q}\right\} \cap\left\{T_{3 d}>T_{3 d, q}\right\} \cap\left\{d S_{3 d}>d S_{3 d, q}\right\}$, in which $Q_{d y, q}, P_{3 d, q}, T_{3 d, q}$, and $d S_{3 d, q}$ are the $q$ quantile of $Q_{d y}, P_{3 d}, T_{3 d}$, and $d S_{3 d}$. During the selection, events within \pm 3 days of other selected events were not selected. The process stopped when a total number of $(r \times$ years $)$ events was identified. Clearly, the quantile levels do not need to be the same for each variable. However, to simplify the case study, the same quantile level was used for consistency across all variables. Other M2 examples were examined by Kew et al. (2013) and Bevacqua et al. (2019).

- M3 - maximum joint empirical probability: In this approach, the cumulative joint empirical probability of each data point $\left(q_{d y}, p_{3 d}, t_{3 d}, d s_{3 d}\right)$ (i.e., $\mathrm{P}\left[\left\{Q_{d y} \leq q_{d y}\right\} \cap\left\{P_{3 d} \leq p_{3 d}\right\} \cap\left\{T_{3 d} \leq t_{3 d}\right\} \cap\left\{d S_{3 d} \leq\right.\right.$ $\left.\left.d s_{3 d}\right\}\right]$ ) was calculated. Then, an average sampling rate $r$ was set up, and the top ( $r \times$ years) events with higher cumulative empirical probability values were selected. Similar to in M2, events within \pm 3 days of other selected larger events were not selected. A similar method was used and discussed by Kao and Govindaraju (2007).

To focus on snowmelt-related events, the search was further limited to the months of April through June. With 36 years of data (1980-2015), a sampling rate of an average of 5 events per year was selected for both M2 and M3 so that 180 maximum events per method could be gathered for analysis. An example of $Q_{d y}$ versus $d S_{3 d}$ for $\mathrm{S} 2$ is shown in Figure 2-4. Among the three methods, both M2 and M3 look more similar to each other than either one to M1, with the only difference being in the lower corner of both variables. Given the design, M1 selected less events. It did not capture any $d S_{3 d}>45 \mathrm{~mm}$, and also missed many large $Q_{d y}$ likely in wetter years. This could cause some issues during multivariate frequency analysis that are demonstrated in the following sections.
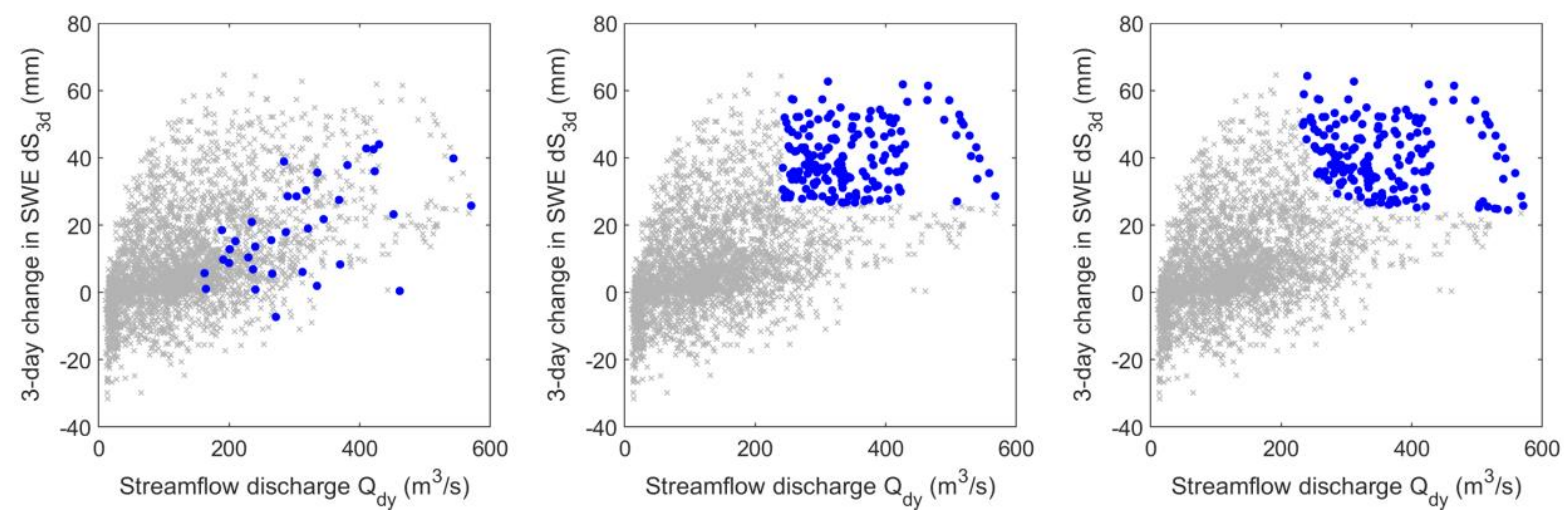

Figure 2-4. An example of maximum event selection (left: M1; middle: M2; right: M3) for S2. The grey crosses show all data points and blue circles show the selected maximum events by each method.

\subsection{FITTING AND SELECTION OF MARGINAL DISTRIBUTIONS}

For copula-based multivariate analysis, the first step is to determine the most suitable $\operatorname{PDF} f(x)$ and cumulative distribution function (CDF) $F(x)$ for each variable (i.e., marginal distributions). Because this step can be achieved through conventional univariate frequency analysis, many existing tools and methods can be used to help select distributions and estimate their parameters. In this case study, the MATLAB statistical toolbox was used. Five commonly used distributions are tested, including logPearson type III (LP3), generalized extreme value (GEV), lognormal (LN), gamma (GM), and normal (NOR) distributions. Rao and Hamed (2000), as well as other statistical analysis textbooks, provided the theoretical background of univariate analysis. Model parameters were estimated by the maximum likelihood method. 
To assess the goodness-of-fit, KS and CM tests at a 5\% significance level were used. Additionally, the fitted $\operatorname{CDF} F(x)$ was compared with the empirical $\operatorname{CDF} F_{e m p}(x)$ derived from the Gringorton plotting position formula:

$$
F_{e m p}(x)=\frac{m-0.44}{n+0.12}
$$

where $n$ is the total number of samples, and $m$ is the rank in the ascending order. For the selection of a suitable distribution, AIC (Akaike 1973) and BIC (Schwarz 1978) were used to help select a suitable distribution. Their formulations are as follows:

$$
\begin{gathered}
\mathrm{AIC}=-2 \times(\text { log-likelihood })+2 \times(\text { numParam }), \\
\mathrm{BIC}=-2 \times(\text { log-likelihood })+\text { numParam } \times \log (\text { numObs }),
\end{gathered}
$$

where log-likelihood refers to the log of likelihood function that can be calculated by the product of PDF values with fitted parameters across all samples, numParam is the number of parameters in a PDF, and numObs is the number of observations. In general, AIC and BIC consider the joint effects of fitting (likelihood) and the number of parameters. A smaller AIC and/or BIC value suggests better performance of a selected PDF. Additionally, the differences between fitted and empirical CDFs on the hazard curves were compared to help determine the most suitable PDF. The results of marginal distributions were compared first across different maximum events and then across different study areas.

\subsubsection{Comparison across different maximum events}

Taking S2 as an example, the five distributions were fitted to three sets of maximum events of each variable. If a distribution failed to pass $\mathrm{KS}$ and $\mathrm{CM}$ tests, they are excluded from further consideration. The resulting AIC and BIC values of each fitted distribution are summarized in Table 2-2.

To help visualize the differences among fitted CDFs, hazard curves (i.e., magnitude of extremes versus return periods) of each variable were plotted. For a random variable $X$ with $T$-year return period, $x(T)$ can be calculated by

$$
x(T)=F^{-1}\left(1-\frac{1}{T * r}\right)
$$

where $F^{-1}$ is the inverse CDF, $T$ is the return period in years, and $r$ is the average sampling rate. In this case study, $r=1$ for M1 events, and $r=5$ for M2 and M3 events. 
Table 2-2. Fitting of marginal distributions using three maximum event searching approaches for S2 (USGS ID: 06191500).

\begin{tabular}{|c|c|c|c|c|c|c|c|c|}
\hline & \multicolumn{2}{|c|}{ Streamflow $\left(Q_{d y}\right)$} & \multicolumn{2}{|c|}{ Precipitation $\left(P_{3 d}\right)$} & \multicolumn{2}{|c|}{ Temperature $\left(T_{3 d}\right)$} & \multicolumn{2}{|c|}{ SWE change $\left(d S_{3 d}\right)$} \\
\hline & AIC & BIC & AIC & BIC & AIC & BIC & AIC & BIC \\
\hline \multicolumn{9}{|c|}{ M1 Univariate Maximum } \\
\hline LP3 & - & - & - & - & - & - & - & - \\
\hline GEV & 693.3 & 698.0 & 250.4 & 255.1 & 183.7 & 188.5 & 296.9 & 301.7 \\
\hline NOR & 695.4 & 698.5 & 266.8 & 270.0 & 184.9 & 188.0 & 296.3 & 299.4 \\
\hline LN & 691.1 & 694.2 & - & - & 182.4 & 185.6 & - & - \\
\hline GM & 691.6 & 694.7 & - & - & 181.0 & 184.2 & - & - \\
\hline \multicolumn{9}{|c|}{ M2 Multivariate Peak-over-threshold } \\
\hline LP3 & $3,362.2$ & $3,371.8$ & - & - & - & - & - & - \\
\hline GEV & $3,362.0$ & $3,371.6$ & 985.7 & 995.3 & 770.9 & 780.5 & $1,298.4$ & $1,308.0$ \\
\hline NOR & - & - & - & - & 787.6 & 794.0 & - & - \\
\hline LN & $3,368.9$ & $3,375.2$ & 985.4 & 991.8 & 767.9 & 774.3 & $1,286.9$ & $1,293.3$ \\
\hline GM & $3,382.2$ & $3,388.6$ & 996.6 & $1,003.0$ & 770.3 & 776.7 & $1,289.2$ & $1,295.6$ \\
\hline \multicolumn{9}{|c|}{ M3 Maximum Joint Empirical Probability } \\
\hline LP3 & $3,430.7$ & $3,440.3$ & - & - & - & - & - & - \\
\hline GEV & $3,431.5$ & $3,441.1$ & $1,251.6$ & $1,261.2$ & 800.5 & 810.1 & $1,339.1$ & $1,348.7$ \\
\hline NOR & - & - & - & - & 803.1 & 809.5 & - & - \\
\hline LN & $3,429.8$ & $3,436.2$ & $1,249.5$ & $1,255.9$ & - & - & $1,337.1$ & $1,343.5$ \\
\hline GM & $3,435.7$ & $3,442.1$ & $1,251.5$ & $1,257.9$ & 800.7 & 807.1 & $1,324.5$ & $1,330.9$ \\
\hline
\end{tabular}

The hazard curves of daily streamflow $\left(Q_{d y}\right)$ are shown in Figure 2-5. They are visualized in semi-log scale to emphasize the fitting of the right tail. In terms of the general selection process, KS and CM tests were first used to remove unsuitable PDFs. Then, AIC and BIC were used to identify better-fitted PDFs, and finally, hazard curves were used to help determine suitable PDFs. For M1, LN and GM provide better fits than GEV and NOR based on AIC and BIC. The hazard curves further suggest that GM may provide a better fit of the right tail. For M2, LP3 and GEV provide relatively better fits based on AIC and BIC. The hazard curves further suggest that LP3 may provide a better fit of the right tail. For M3, LP3 and LN provide relatively better fits based on AIC and BIC. Although the hazard curves show that LN may provide a slightly better fit than LP3, LP3 was eventually selected because of its overall good fitting of peak streamflow across multiple sites and maximum searching methods. 

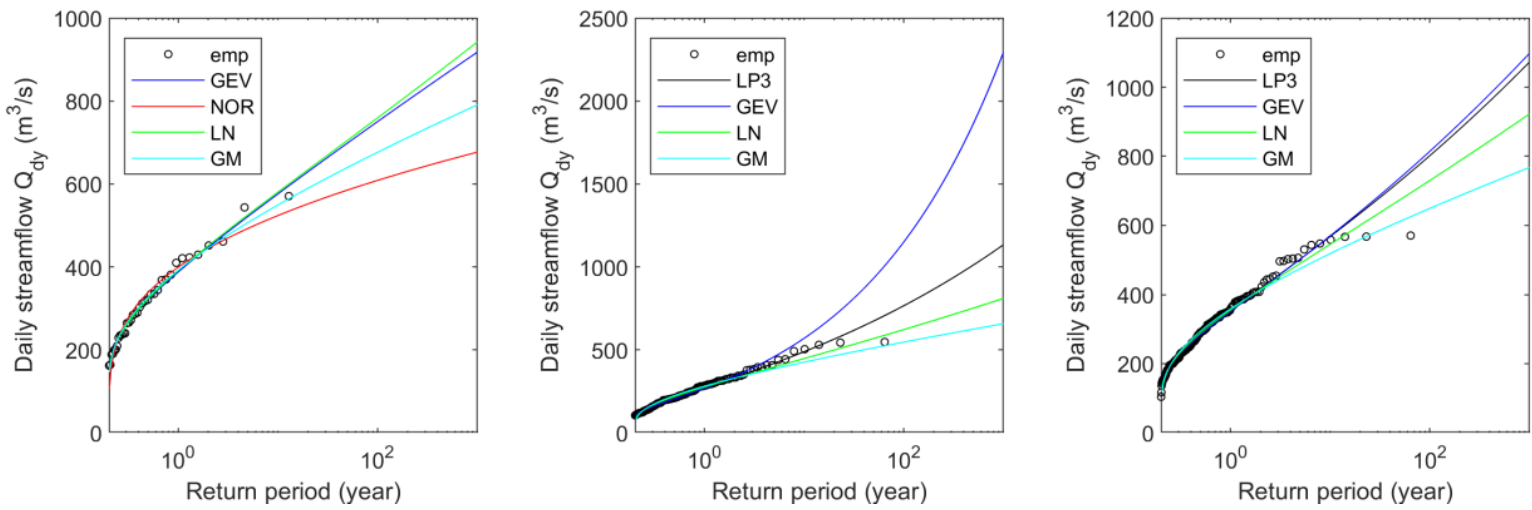

Figure 2-5. Fitting of $Q_{d y}$ using maximum events identified by (left) M1, (middle) M2, and (right) M3 for S2.

Given the nature of the annual maximum searching approach, the sample size of M1 is much smaller than M2 and M3. This size explains why M1 tends to provide smaller estimates than M2 and M3. Also, although LP3 has been a widely accepted distribution for streamflow, suitable LP3 parameters cannot be found for M1. Again, this could be because of the smaller sample size of M1. Although LP3 did not provide the lowest AIC and BIC values for M2 and M3, it overall performed well and seemed to have a more reasonable tail behavior in M2. Since LP3 is commonly used for peak streamflow analysis, it is a good choice to model $Q_{d y}$ in M2 and M3.

Hazard curves for 3-day total precipitation $\left(P_{3 d}\right)$ are shown in Figure 2-6. For M1, NOR provides the relative best fit. Because M1 events were selected based on the annual maximum $Q_{d y}$, the corresponding $P_{3 d}$ could sometimes be small and makes the fitting more challenging. For M2 and M3, GEV and LN provide the relative best fits. Because $\mathrm{LN}$ has a more reasonable tail behavior in M2, LN is a better choice for both M2 and M3. The hazard curves of 3-day average temperature $\left(T_{3 d}\right)$ are shown in Figure 2-7. For M1, GM provides the relative best fit. Although the hazard curves also suggest that GEV may provide good fits of the right tail, its overall fitting is relatively poor and thus affected its AIC and BIC values. For M2, both LN and GEV provide a good fit, and for M3, GEV provides the best fit. Overall, the M1 results are more different than M2 and M3. Again, this difference may be caused by their different sample sizes. Finally, the hazard curves of the decrease of 3-day SWE reduction $\left(d S_{3 d}\right)$ are shown in Figure 2-8. For M1, both GEV and NOR provide relative better fits, and for M2 and M3, GM provides the relative best fit.
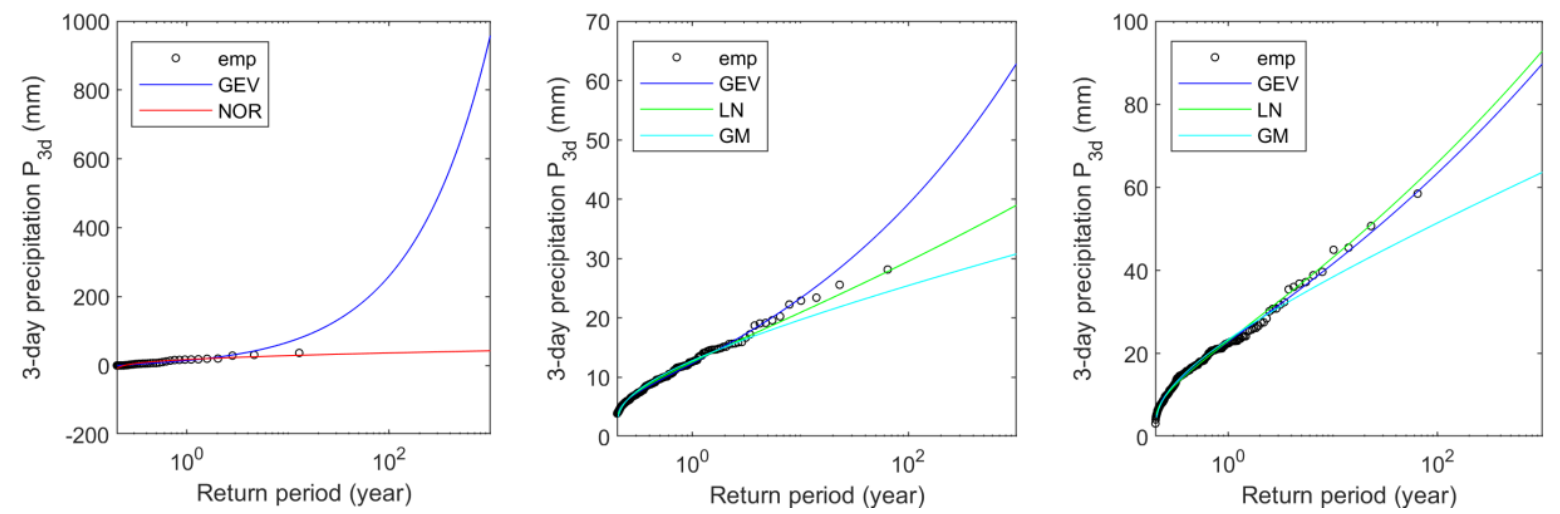

Figure 2-6. Fitting of $P_{3 d}$ using maximum events identified by (left) M1, (middle) M2, and (right) M3 for S2. 

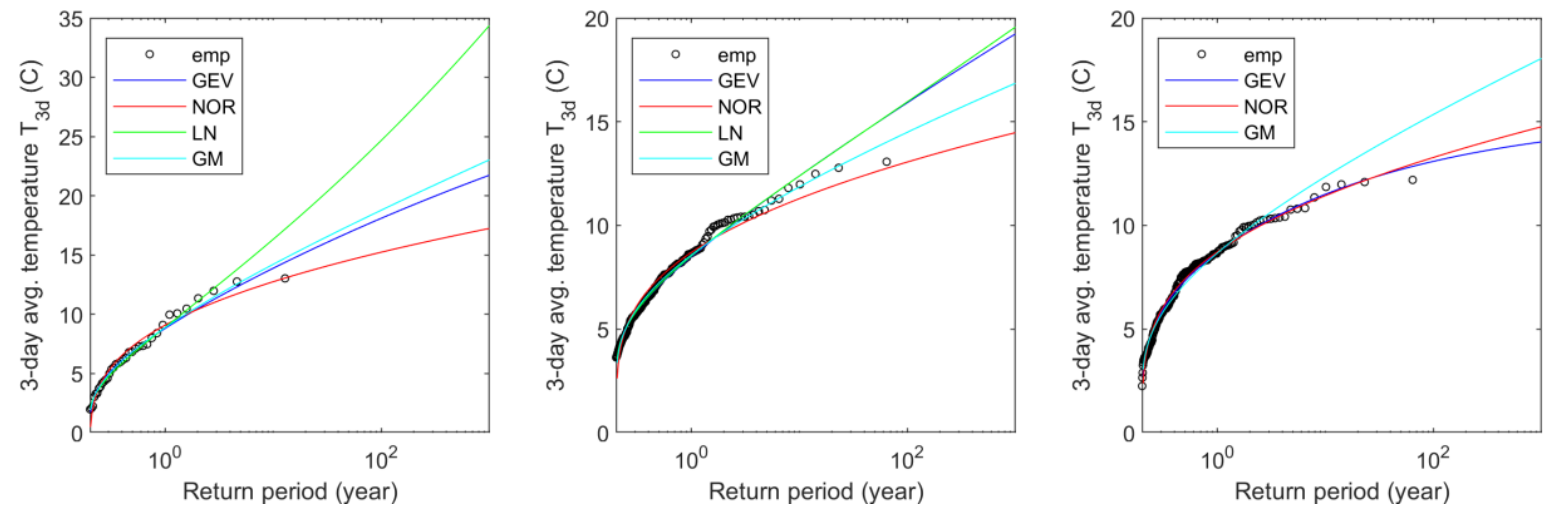

Figure 2-7. Fitting of $T_{3 d}$ using maximum events identified by (left) M1, (middle) M2, and (right) M3 for S2.
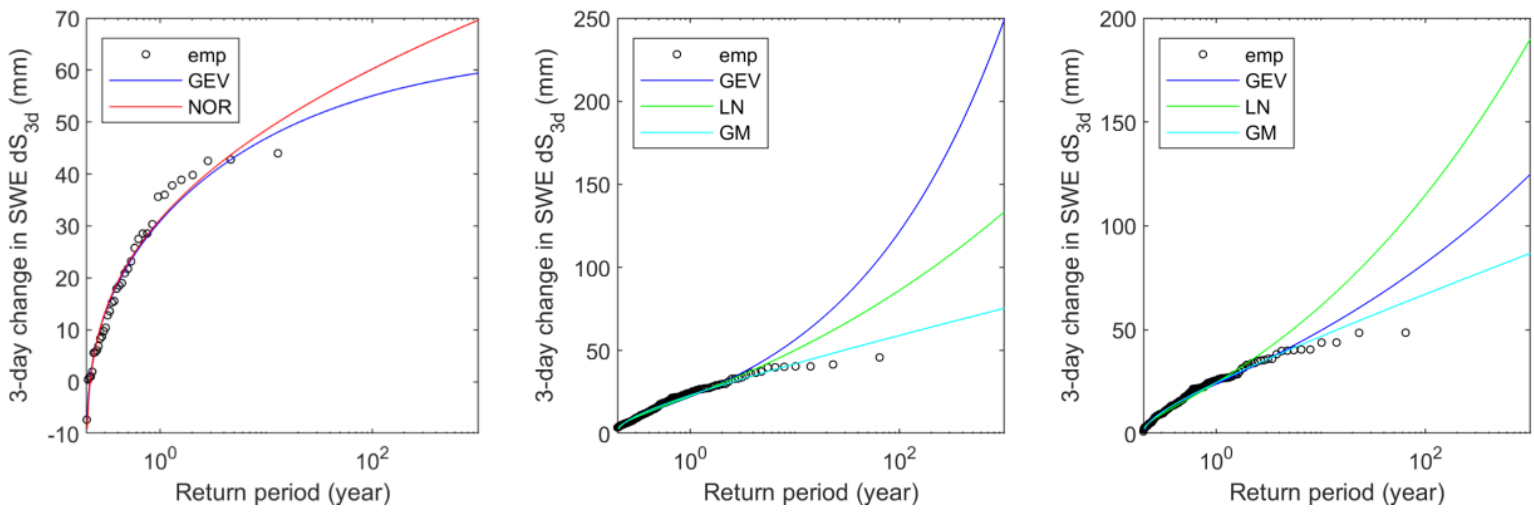

Figure 2-8. Fitting of $d S_{3 d}$ using maximum events identified by (left) M1, (middle) M2, and (right) M3 for S2.

\subsubsection{Comparison across different study areas}

This section shows the difference of marginal distributions across the three study areas. Taking M2 maximum events as an example, the five distributions were fitted across S1-S3 study areas. If a distribution failed to pass KS and CM tests, it was excluded from further consideration. The resulting AIC and BIC values are summarized in Table 2-3. 
Table 2-3. Fitting of marginal distributions using M2 for the three study areas.

\begin{tabular}{|c|c|c|c|c|c|c|c|c|}
\hline & \multicolumn{2}{|c|}{ Streamflow $\left(Q_{d y}\right)$} & \multicolumn{2}{|c|}{ Precipitation $\left(P_{3 d}\right)$} & \multicolumn{2}{|c|}{ Temperature $\left(T_{3 d}\right)$} & \multicolumn{2}{|c|}{ SWE Change $\left(d S_{3 d}\right)$} \\
\hline & AIC & BIC & AIC & BIC & AIC & BIC & AIC & BIC \\
\hline \multicolumn{9}{|c|}{ S1 Clearwater River at Orofino, ID (USGS ID: 13340000) } \\
\hline LP3 & $3,593.4$ & $3,603.0$ & - & - & 794.0 & 803.5 & - & - \\
\hline GEV & $3,595.7$ & $3,605.2$ & $1,070.8$ & $1,080.4$ & 792.7 & 802.3 & $1,151.6$ & $1,161.2$ \\
\hline NOR & - & - & $1,116.5$ & $1,122.9$ & - & - & - & - \\
\hline $\mathrm{LN}$ & $3,626.5$ & $3,632.9$ & $1,066.7$ & $1,073.1$ & 803.3 & 809.7 & $1,157.4$ & $1,163.8$ \\
\hline GM & - & - & $1,070.3$ & $1,076.7$ & 811.3 & 817.7 & - & - \\
\hline \multicolumn{9}{|c|}{ S2 Yellowstone River at Corwin Springs, MT (USGS ID: 06191500) } \\
\hline LP3 & $3,362.2$ & $3,371.8$ & - & - & - & - & - & - \\
\hline GEV & $3,362.0$ & $3,371.6$ & 985.7 & 995.3 & 770.9 & 780.5 & $1,298.4$ & $1,308.0$ \\
\hline NOR & - & - & - & - & 787.6 & 794.0 & - & - \\
\hline $\mathrm{LN}$ & $3,368.9$ & $3,375.2$ & 985.4 & 991.8 & 767.9 & 774.3 & $1,286.9$ & $1,293.3$ \\
\hline GM & $3,382.2$ & $3,388.6$ & 996.6 & $1,003.0$ & 770.3 & 776.7 & $1,289.2$ & $1,295.6$ \\
\hline \multicolumn{9}{|c|}{ S3 NF Clearwater River NR Canyon Ranger Station, ID (USGS ID: 13340600) } \\
\hline LP3 & $3,308.8$ & $3,318.4$ & - & - & 772.7 & 782.3 & - & - \\
\hline GEV & $3,309.9$ & $3,319.5$ & $1,151.2$ & $1,160.8$ & 772.2 & 781.8 & - & - \\
\hline NOR & - & - & - & - & - & - & - & - \\
\hline $\mathrm{LN}$ & $3,342.0$ & $3,348.4$ & $1,145.6$ & $1,151.9$ & 790.4 & 796.8 & - & - \\
\hline GM & - & - & $1,154.4$ & $1,160.8$ & 801.9 & 808.3 & $1,441.8$ & $1,448.2$ \\
\hline
\end{tabular}

The hazard curves of daily streamflow $\left(Q_{d y}\right)$ are shown in Figure 2-9. Despite the different site characteristics, the fitting results here are generally consistent across the three study areas. For all sites, LP3 provides the best (or nearly best) AIC and BIC values. The tail behaviors of LP3 are more reasonable than GEV in the hazard curves, which is consistent with the general understanding that LP3 is a suitable distribution for peak streamflow analysis. Therefore, LP3 is a good choice to model M2 $Q_{d y}$ events across all sites.
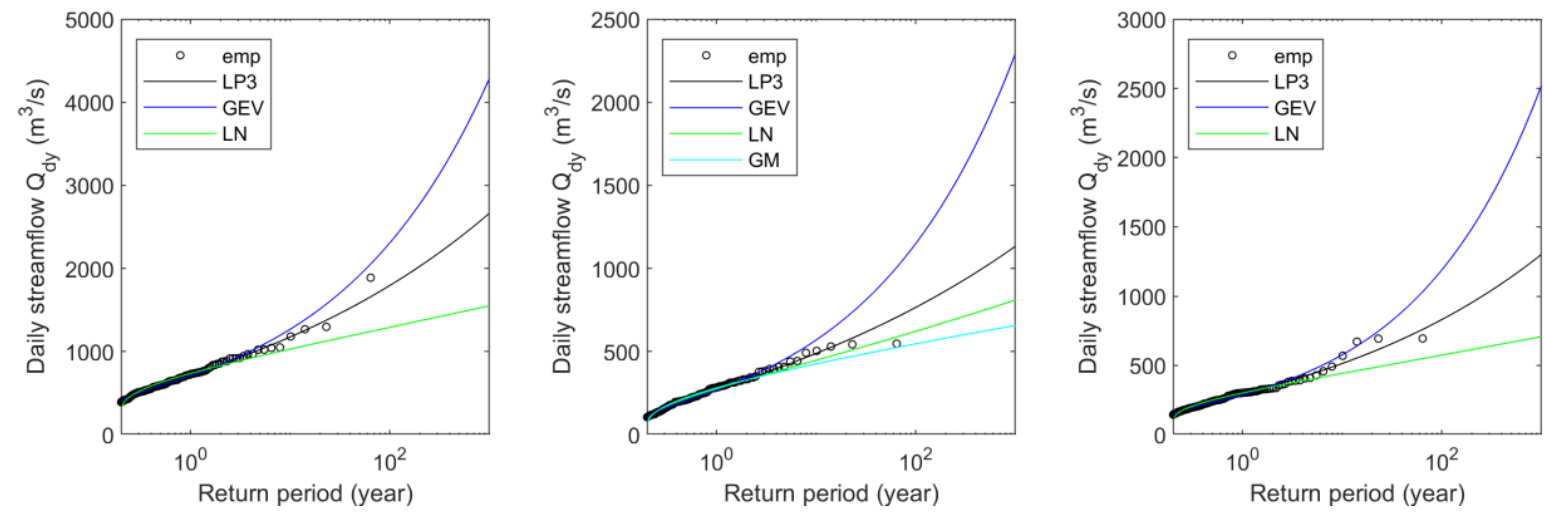

Figure 2-9. Fitting of $Q_{d y}$ using M2 maximum events for (left) S1, (middle) S2, and (right) S3.

The hazard curves of 3-day total precipitation $\left(P_{3 d}\right)$ are shown in Figure 2-10. Again, despite different site characteristics, LN performs the best across the three sites and is a suitable choice for follow-up analysis. The hazard curves of 3-day average temperature $\left(T_{3 d}\right)$ are shown in Figure 2-11. Based on the AIC and 
BIC values and hazard curves, both GEV and LN provide good fits. The hazard curves of the 3-day SWE reduction $\left(d S_{3 d}\right)$ are shown in Figure 2-12. For S1, LN provides the best fit, whereas for S2 and S3, GM provides the best fit.
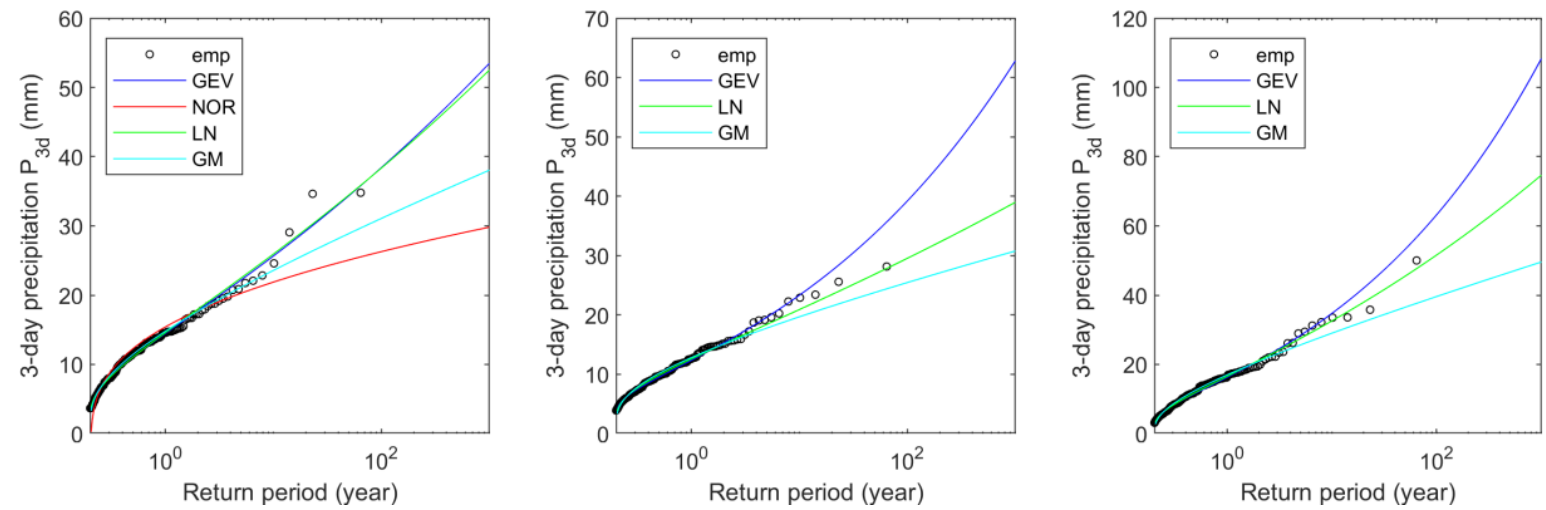

Figure 2-10. Fitting of $P_{3 d}$ using M2 maximum events for (left) S1, (middle) S2, and (right) S3.
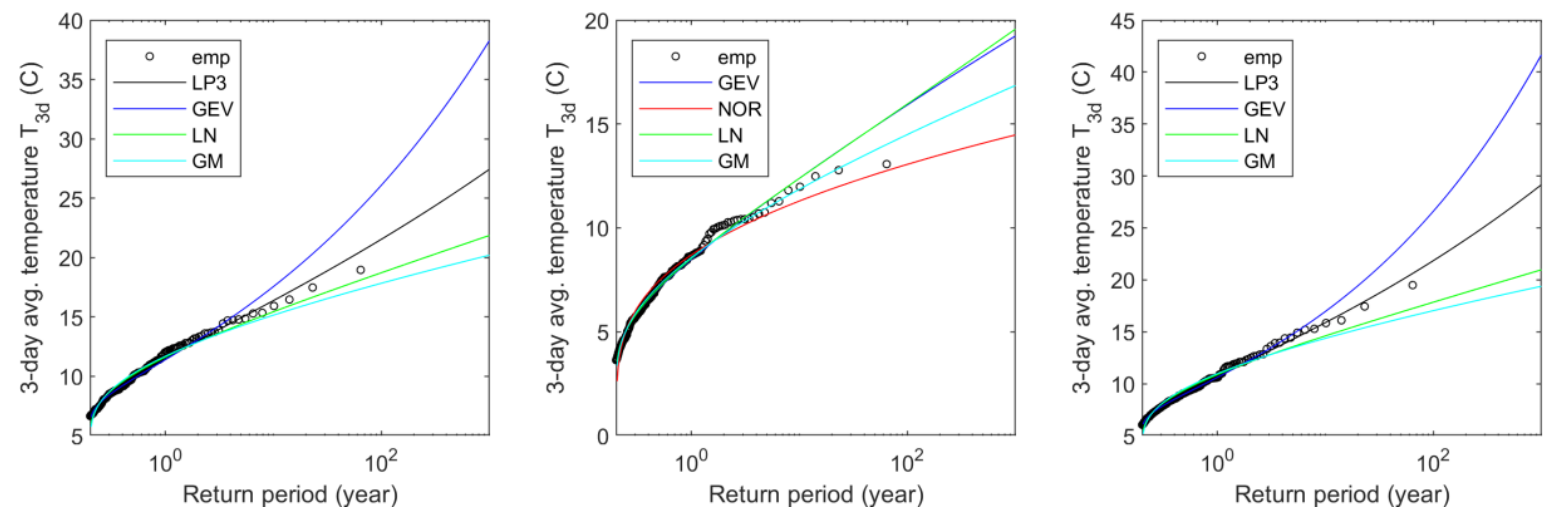

Figure 2-11. Fitting of $T_{3 d}$ using M2 maximum events for (left) S1, (middle) S2, and (right) S3.
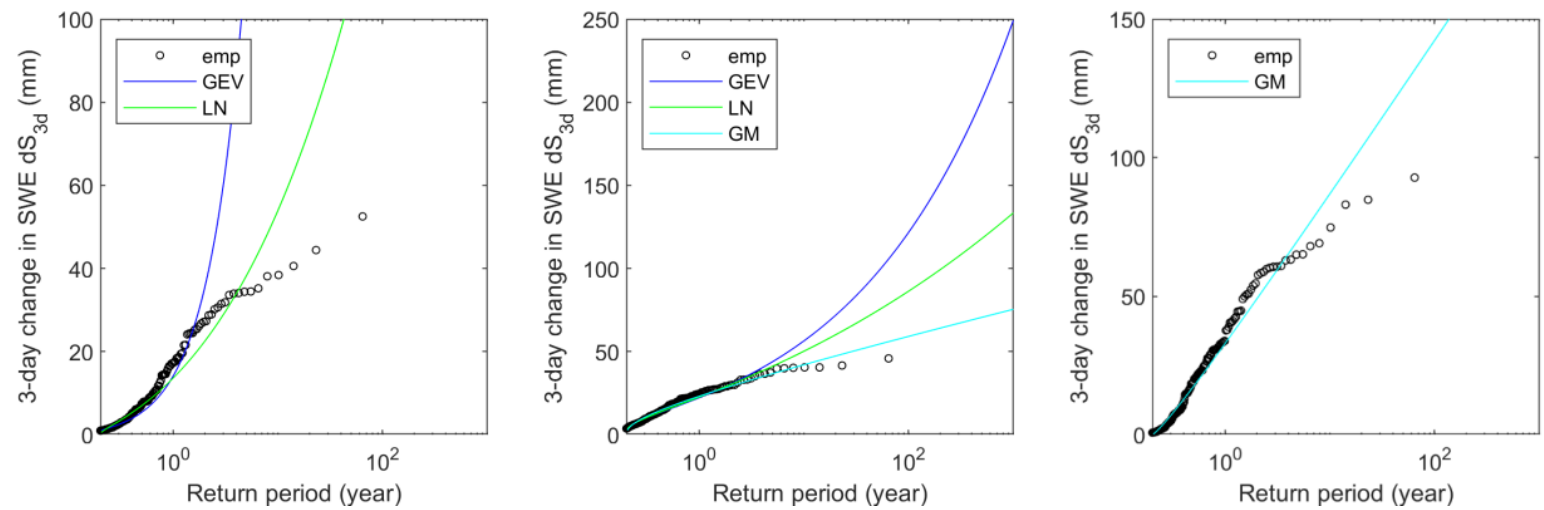

Figure 2-12. Fitting of $d S_{3 d}$ using M2 maximum events for (left) S1, (middle) S2, and (right) S3.

Overall, different maximum event searching approaches seem to have more profound effects than different sites. The results of annual maximum-based M1 events are more different than peak-overthreshold-based M2 and M3 events. This difference can be largely affected by the number of samples, in which five times larger samples are allowed in M2 and M3 so that they may capture more larger events in 
a wetter (or hotter) year. More importantly, because M1 only identifies the maximum $Q_{d y}$ events, other variables can be quite small and lead to lower estimates than M2 and M3. The smaller sample size of M1 is also too limited to support multivariate analysis. Because of various disadvantages, M1 was excluded in the following analysis. The selected marginal distributions and their parameters are summarized in Table $2-4$.

Table 2-4. Selected marginal distribution and fitted parameters.

\begin{tabular}{l|c|c|c|c}
\hline \multicolumn{1}{c|}{ Variables } & \multicolumn{1}{c|}{$\begin{array}{c}\text { Selected } \\
\text { distribution }\end{array}$} & $\begin{array}{c}\text { Location } \\
\text { parameter }(\boldsymbol{\mu})\end{array}$ & $\begin{array}{c}\text { Scale } \\
\text { parameter }(\boldsymbol{\alpha})\end{array}$ & $\begin{array}{c}\text { Shape } \\
\text { parameter }(\boldsymbol{\xi})\end{array}$ \\
\hline \multicolumn{5}{|c|}{ S1 Clearwater River at } \\
\hline Strefino, ID; M2 Multivariate Peak-over-threshold \\
\hline Precipitation $\left(P_{3 d}\right)$ & LP3 & 5.8112 & 0.1357 & 4.1249 \\
\hline Temperature $\left(T_{3 d}\right)$ & LN & 2.28162 & 0.474342 & - \\
\hline SWE Change $\left(d S_{3 d}\right)$ & LN & 2.25578 & 0.234191 & - \\
\hline
\end{tabular}

S2 Yellowstone River at Corwin Springs, MT; M2 Multivariate Peak-over-threshold

\begin{tabular}{l|c|c|c|c}
\hline Streamflow $\left(Q_{d y}\right)$ & LP3 & 3.4186 & 0.0853 & 21.8906 \\
\hline Precipitation $\left(P_{3 d}\right)$ & LN & 2.1799 & 0.419003 & - \\
\hline Temperature $\left(T_{3 d}\right)$ & LN & 1.88482 & 0.307596 & - \\
\hline SWE Change $\left(d S_{3 d}\right)$ & GM & 2.61021 & 6.12062 & - \\
\hline
\end{tabular}

S2 Yellowstone River at Corwin Springs, MT; M3 Maximum Joint Empirical Probability

\begin{tabular}{l|c|c|c|c}
\hline Streamflow $\left(Q_{d y}\right)$ & LP3 & 4.7865 & 0.1525 & 4.3023 \\
\hline Precipitation $\left(P_{3 d}\right)$ & LN & 2.33897 & 0.557574 & - \\
\hline Temperature $\left(T_{3 d}\right)$ & GEV & 7.91694 & 1.54965 & 0.194182 \\
\hline SWE Change $\left(d S_{3 d}\right)$ & GM & 0.814919 & 24.8653 & - \\
\hline
\end{tabular}

S3 NF Clearwater River NR Canyon Ranger Station, ID; M2 Multivariate Peak-over-threshold

\begin{tabular}{l|c|c|c|c}
\hline Streamflow $\left(Q_{d y}\right)$ & LP3 & 2.3983 & 0.0392 & 81.1015 \\
\hline Precipitation $\left(P_{3 d}\right)$ & LN & 2.70661 & 0.515288 & - \\
\hline Temperature $\left(T_{3 d}\right)$ & GEV & 6.0053 & 2.11848 & -0.225228 \\
\hline SWE Change $\left(d S_{3 d}\right)$ & GM & 2.18285 & 7.61097 & - \\
\hline
\end{tabular}

Note: For LP3 and GEV, parameter 1 is the location parameter $(\mu)$, parameter 2 is the scale parameter $(\alpha)$, and parameter 3 is the shape parameter $(\xi)$. For LN, parameter 1 is the location parameter $(\mu)$, and parameter 2 is the scale parameter $(\alpha)$. For GM, parameter 1 is the shape parameter $(\alpha)$, and parameter 2 is the rate parameter $(\beta)$.

\subsection{FITTING AND SELECTION OF COPULA FUNCTIONS}

Following the selection of marginal distributions, the second step is to determine the most suitable copula function $C_{U V}(u, v)$ that can couple marginal distributions into a joint probability distribution $F_{X Y}(x, y)$. General mathematical expressions for copula cumulative distribution and density functions are

$$
\begin{gathered}
F_{X Y}(x, y)=C_{U V}\left(u=F_{X}(x), v=F_{Y}(y)\right)=C_{U V}(u, v) \\
f_{X Y}(x, y)=\frac{\partial u}{\partial x} \frac{\partial v}{\partial y} \frac{\partial^{2} C_{U V}(u, v)}{\partial u \partial v}=f_{X}(x) f_{Y}(y) c_{U V}(u, v)
\end{gathered}
$$

where $F_{X Y}(x, y)$ is the joint-CDF (JCDF), and $f_{X Y}(x, y)$ is the joint-PDF of variables $X$ and $Y, u=F_{X}(x)$ is the marginal distribution of $X, v=F_{Y}(y)$ is the marginal distribution of $Y, C_{U V}(u, v)$ is the copula 
function, and $c_{U V}(u, v)$ is the copula density function. Equation (2.5) can be interpreted as a transformation of JCDF from the $X Y$ to $U V$ domain. Because $U$ and $V$ are uniformly distributed after this transformation, the copula function is hence marginal free and only retains information related to the dependence structure among two variables. This important feature allows one to sperate any JCDF into two components - marginal distributions and dependence structure. One can identify the most suitable mathematical expressions of both components and then combine them to form a JCDF. The approach is flexible and compatible to most existing JCDF models, such as bivariate Gaussian and exponential distributions. Nelsen (2006) provided the theoretical background of copulas.

Although the concept of copulas is relatively new compared with conventional univariate frequency analysis, the procedure is conceptually similar. It starts by selecting and fitting different copula functions. In this case study, five commonly used copula functions were tested: Gaussian (GAU), $t$ with degree of freedom = 2 (TD2), Frank (FRK), Clayton (CLT), and Gumbel (GUM). The first two copulas (GAU and TD2) belong to the family of meta-elliptical copulas, which are transformations of the well-known metaelliptical distributions that include both multivariate Gaussian and t distributions (Genest et al. 2007). The other three copulas (FRK, CLT, and GUM) belong to a special class of Archimedean copulas. For each Archimedean copula, a generator $\phi$ exists such that the following relationship holds:

$$
\phi\left(C_{U V}(u, v)\right)=\phi(u)+\phi(v)
$$

where the generator $\phi$ should be a continuous strictly decreasing function in $[0,1]$ with $\phi(0)=\infty$ and $\phi(1)=0$, and the inverse $\phi^{-1}$ should be strictly monotonic (Nelsen 2006). For Archimedean copulas, several statistical properties can be simply expressed in terms of the generator $\phi$, such as the Kendall's concordance measure $\tau$ :

$$
\tau=1+4 \int_{0}^{1} \frac{\phi(t)}{\phi^{\prime}(t)} d t
$$

An example of Frank Archimedean copulas $\left(\phi(t)=-\ln \frac{e^{-\theta t}-1}{e^{-\theta}-1}\right)$ is shown in Figure 2-13, where the parameter $\theta$ controls the shape of dependence structure between marginals $U$ and $V$. 


\section{Frank family of Archimedean copulas

$$
C_{\text {Frank }}(u, v)=-\frac{1}{\theta} \ln \left(1+\frac{\left(e^{-\theta_{\iota}}-1\right)\left(e^{-\theta v}-1\right)}{e^{-\theta}-1}\right)
$$
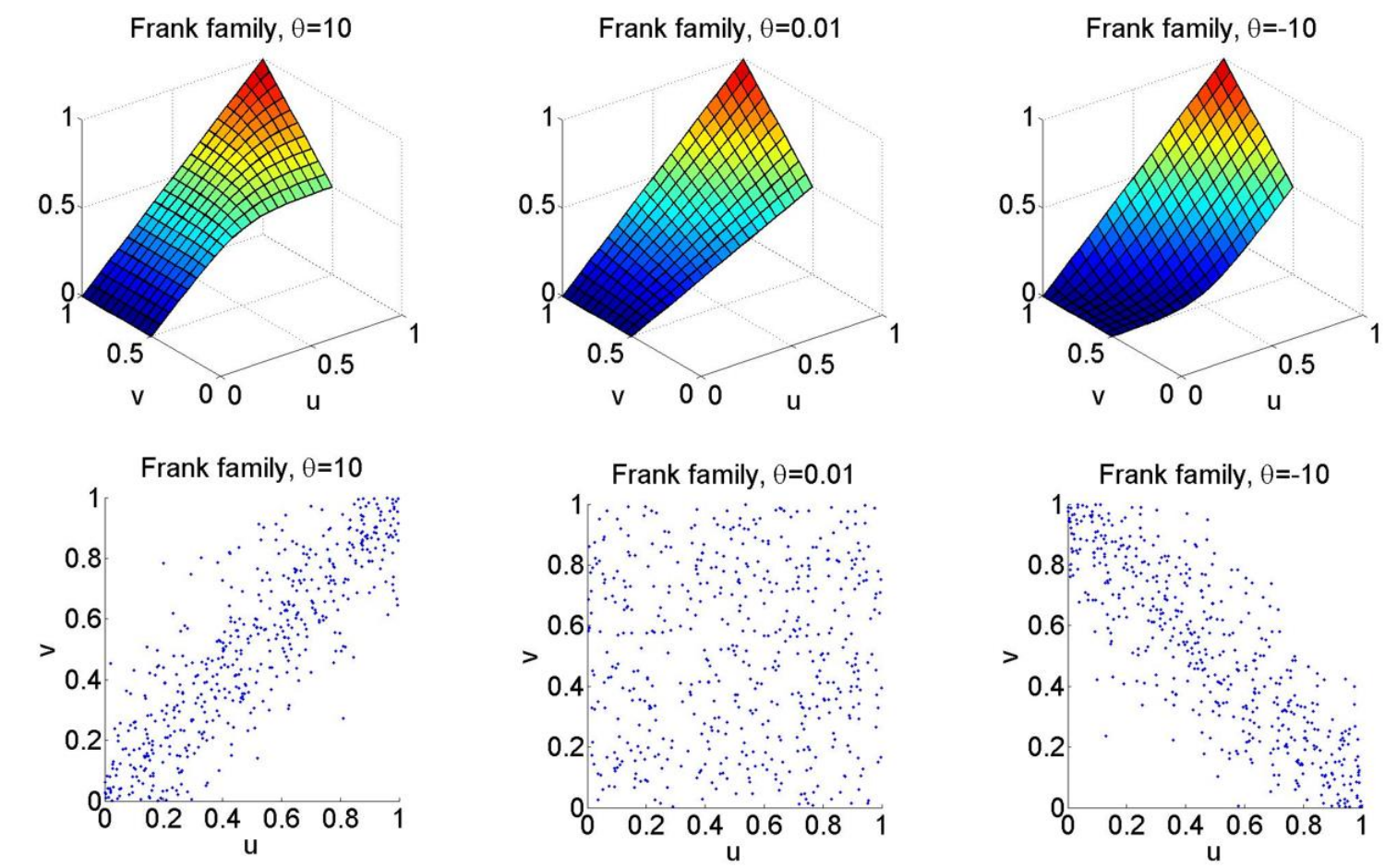

Figure 2-13. An example of the Frank copula function.

For parameter estimation and goodness-of-fit, the rank-based empirical copula $C_{n}$ (Nelsen 2006) can be used:

$$
C_{n}\left(\frac{i}{n}, \frac{j}{n}\right)=\frac{a}{n}
$$

where $n$ is the total number of samples, $a$ is the number of pairs $(x, y)$ with $x \leq x_{(i)}$ and $y \leq y_{(j)}$, and $x_{(i)}, y_{(j)}, 1 \leq i, j \leq n$ is the rank of each sample. To estimate the copula function parameter, one can either select the inference functions for margins approach that uses fitted marginals, or the canonical maximum likelihood approach that uses rank-based empirical marginals to analyze the copulas. Canonical maximum likelihood was selected because it is purely based on samples' rank and is unrelated to the selection of marginal CDFs. Another approach that solves the copula parameter using Kendall's $\tau$ is also widely used for the family of Archimedean copulas (e.g., FRK, CLT, GUM; Kao and Govindaraju 2007).

To assess the goodness-of-fit, the multidimensional KS test (Saunders and Laud 1980) at a 5\% significance level was used. For the selection of a suitable distribution, AIC and BIC (Eqs. 2.2 and 2.3) were used by calculating a likelihood function as the product of copula density values with fitted parameters across all samples. The five selected copula functions were fitted to all four cases reported in Table 2-4, and the resulting AIC and BIC values are reported in Table 2-5. 
Table 2-5. Fitting of copula function for the four selected cases.

\begin{tabular}{|c|c|c|c|c|c|c|}
\hline & \multicolumn{2}{|c|}{ Between $Q_{d y}$ and $P_{3 d}$} & \multicolumn{2}{|c|}{ Between $Q_{d y}$ and $T_{3 d}$} & \multicolumn{2}{|c|}{ Between $Q_{d y}$ and $d S_{3 d}$} \\
\hline & AIC & $\mathrm{BIC}$ & $\mathrm{AIC}$ & $\mathrm{BIC}$ & $\mathrm{AIC}$ & $\mathrm{BIC}$ \\
\hline \multicolumn{7}{|c|}{ S1 Clearwater River at Orofino, ID; M2 Multivariate Peak-over-threshold } \\
\hline GAU & -0.51 & 2.68 & 1.96 & 5.16 & -64.4 & -61.2 \\
\hline $\mathrm{TD} 2$ & 7.40 & 10.59 & 28.68 & 31.87 & -34.5 & -31.3 \\
\hline FRK & 0.27 & 3.46 & 1.86 & 5.05 & -65.1 & -61.9 \\
\hline CLT & 0.28 & 3.47 & 1.94 & 5.14 & -61.8 & -58.6 \\
\hline GUM & -1.54 & 1.65 & 2.00 & 5.19 & -46.9 & -43.7 \\
\hline \multicolumn{7}{|c|}{ S2 Yellowstone River at Corwin Springs, MT; M2 Multivariate Peak-over-threshold } \\
\hline GAU & -5.39 & -2.19 & -8.65 & -5.46 & -40.8 & -37.6 \\
\hline TD2 & -15.2 & -12.0 & -9.09 & -5.90 & -35.5 & -32.3 \\
\hline FRK & -5.46 & -2.27 & -9.64 & -6.45 & -36.0 & -32.8 \\
\hline CLT & -2.03 & 1.15 & -11.2 & -8.01 & -36.5 & -33.3 \\
\hline GUM & -11.3 & -8.15 & -8.12 & -4.93 & -37.3 & -34.1 \\
\hline \multicolumn{7}{|c|}{ S2 Yellowstone River at Corwin Springs, MT; M3 Maximum Joint Empirical Probability } \\
\hline GAU & -3.80 & -0.61 & -5.62 & -2.42 & -35.2 & -32.0 \\
\hline TD2 & 28.8 & 32.0 & 1.15 & 4.34 & -0.90 & 2.30 \\
\hline FRK & -1.47 & 1.72 & -5.47 & -2.27 & -32.4 & -29.2 \\
\hline CLT & 2.00 & 5.19 & -2.02 & 1.18 & -22.0 & -18.8 \\
\hline GUM & 2.00 & 5.19 & -7.55 & -4.36 & -30.2 & -27.0 \\
\hline \multicolumn{7}{|c|}{ S3 NF Clearwater River NR Canyon Ranger Station, ID; M2 Multivariate Peak-over-threshold } \\
\hline GAU & 0.28 & 3.48 & 2.03 & 5.22 & -85.2 & -82.0 \\
\hline TD2 & 10.64 & 13.83 & 10.7 & 13.9 & -57.7 & -54.5 \\
\hline FRK & -0.49 & 2.71 & 2.01 & 5.20 & -79.8 & -76.6 \\
\hline CLT & 1.41 & 4.61 & 1.67 & 4.86 & -73.2 & -70.0 \\
\hline GUM & -0.01 & 3.18 & 2.00 & 5.19 & -69.2 & -66.0 \\
\hline
\end{tabular}

To help visualize the results, the spread in both $X Y$ and $U V$ domains of each pair of variables were plotted with $Q_{d y}$ as shown in Figure 2-14 to Figure 2-17. In addition, Kendall's distribution function $K_{C}$ (Genest and Rivest 1993), which projects the cumulative value of a copula into a single dimension (i.e., CDF of a copula), was plotted to more easily compare the performance of different copula functions. The theoretical formulation and empirical distribution of $K_{C}$ are provided as follows.

$$
\begin{gathered}
K_{C}(t)=P[C(u, v) \leq t], \\
K_{C_{n}}\left(\frac{l}{n}\right)=\frac{b}{n},
\end{gathered}
$$

where $K_{C_{n}}$ is the empirical Kendall's distribution, and $b$ is the number of pairs $(x, y)$ in the samples with the empirical copula values $C_{n}(i / n, j / n) \leq l / n$ (Eq. 2.9). In other words, one may use different $l$ values from 1 to $n$ to calculate the empirical copula values $C_{n}$ and construct $K_{C_{n}}$. 

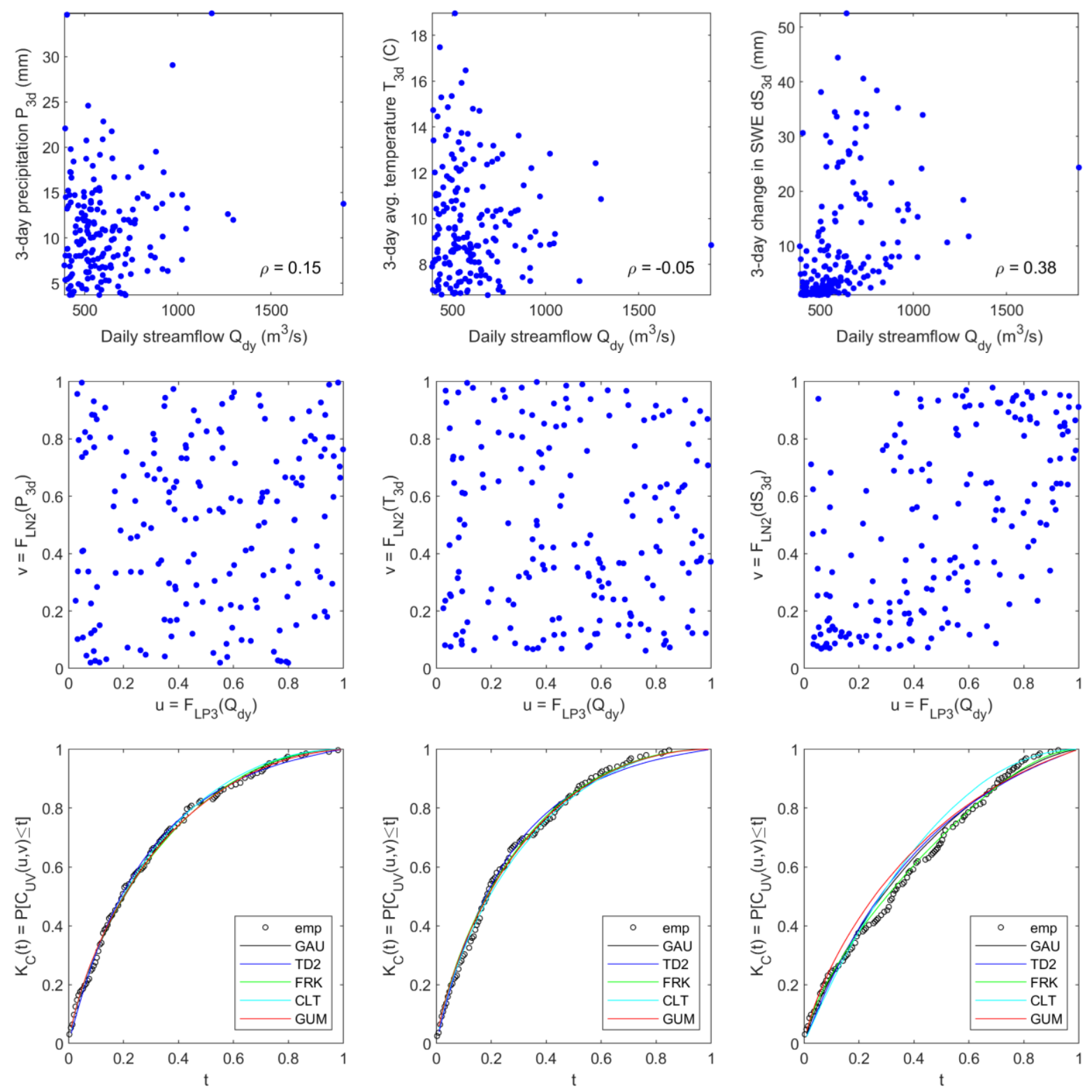

Figure 2-14. Fitting of copulas for S1 using M2 events. The spread in $X Y$ and $U V$ domains are shown in the upper and middle rows. The Kendall's distribution function is shown in the lower row. The results between $Q_{d y}$ and $P_{3 d}$, $Q_{d y}$ and $T_{3 d}$, and $Q_{d y}$ and $d S_{3 d}$ are shown in left, middle, and right columns. 

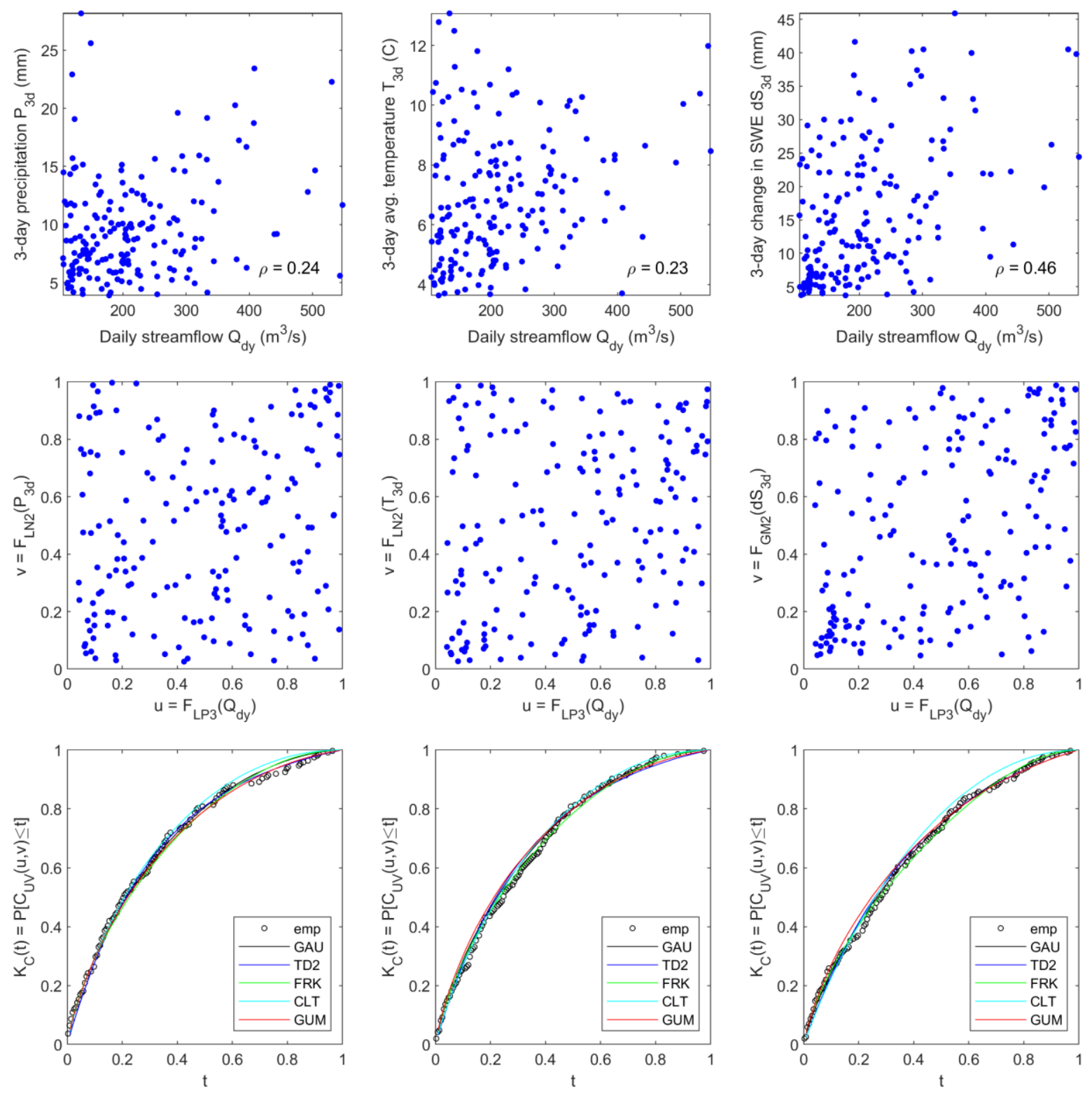

Figure 2-15. Fitting of copulas for $\mathbf{S 2}$ using $\mathbf{M} 2$ events. The spread in $X Y$ and $U V$ domains are shown in the upper and middle rows. The Kendall's distribution function is shown in the lower row. The results between $Q_{d y}$ and $P_{3 d}$, $Q_{d y}$ and $T_{3 d}$, and $Q_{d y}$ and $d S_{3 d}$ are shown in left, middle, and right columns. 

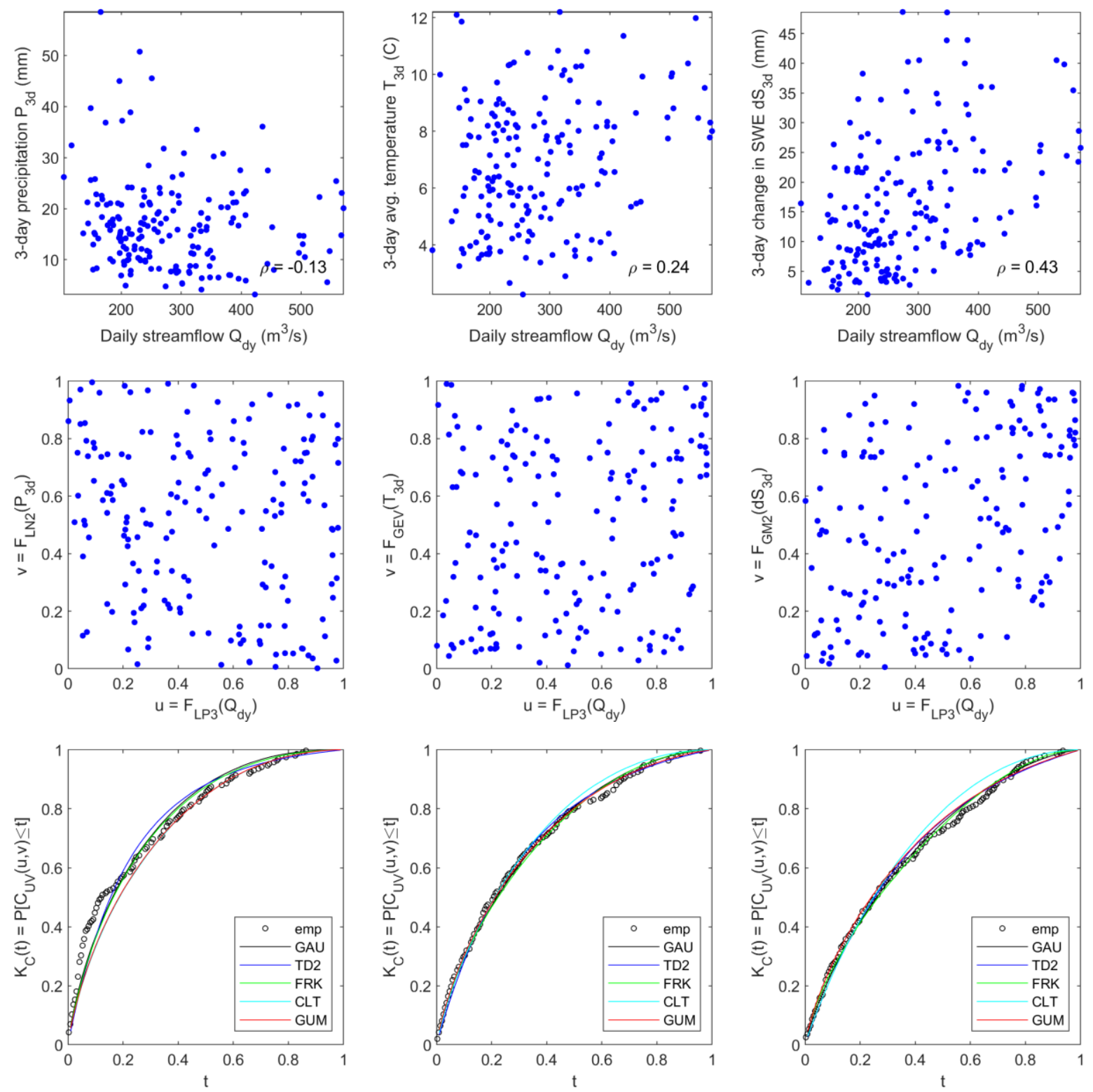

Figure 2-16. Fitting of copulas for $\mathbf{S 2}$ using $\mathbf{M 3}$ events. The spread in $X Y$ and $U V$ domains are shown in the upper and middle rows. The Kendall's distribution function is shown in the lower row. The results between $Q_{d y}$ and $P_{3 d}$, $Q_{d y}$ and $T_{3 d}$, and $Q_{d y}$ and $d S_{3 d}$ are shown in left, middle, and right columns. 

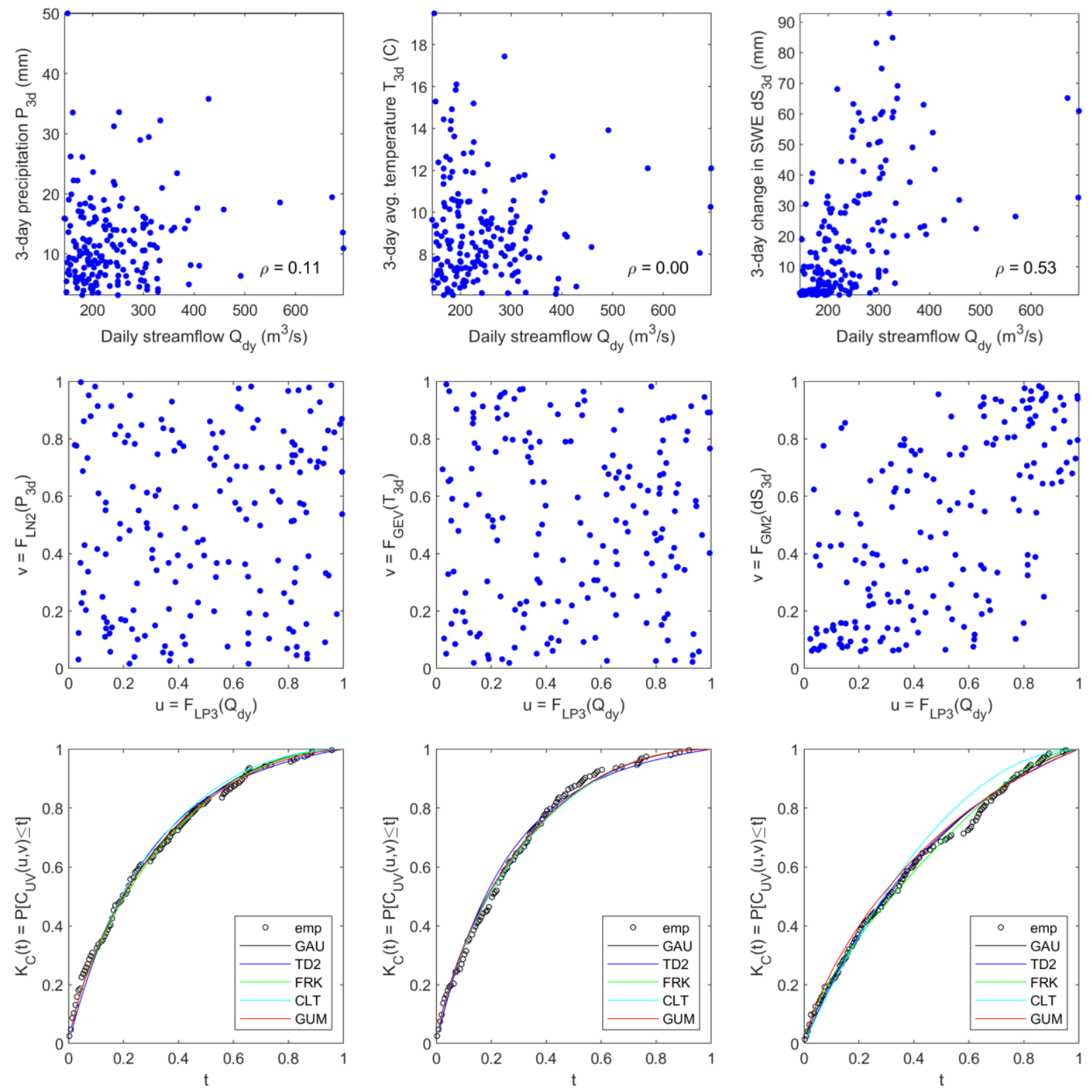

Figure 2-17. Fitting of copulas for $\mathbf{S 3}$ using $\mathbf{M} 2$ events. The spread in $X Y$ and $U V$ domains are shown in the upper and middle rows. The Kendall's distribution function is shown in the lower row. The results between $Q_{d y}$ and $P_{3 d}$, $Q_{d y}$ and $T_{3 d}$, and $Q_{d y}$ and $d S_{3 d}$ are shown in left, middle, and right columns.

Overall, the results suggest that the strongest correlation/dependence existed between $Q_{d y}$ and $d S_{3 d}$, with a correlation coefficient ranging from 0.40 to 0.53 . The correlation between other pairs of variables were weaker and were both site- and method-specific. In some cases, slightly negative correlations were also found. In terms of suitable copula functions, none was determined to be the best. Best-performing copulas were identified among GAU, FRK, CLT, and GUM. In terms of goodness-of-fit, all cases passed the multivariate KS test at a 5\% significant level, and most of the cases showed reasonable fit on the $K_{C}$ plot. Because the multivariate KS test and $K_{C}$ plots were less discriminating, AIC and BIC values were heavily relied upon in selecting suitable copulas. The selected copulas and their parameters are summarized in Table 2-6. 
Table 2-6. Selected copula functions and fitted parameters.

\begin{tabular}{|c|c|c|c|c|}
\hline Variables & $\begin{array}{c}\text { Correlation } \\
\text { coefficient }(\rho)\end{array}$ & Kendall's $\tau$ & $\begin{array}{l}\text { Selected copula } \\
\text { function }\end{array}$ & Copula parameter \\
\hline \multicolumn{5}{|c|}{ S1 Clearwater River at Orofino, ID; M2 Multivariate Peak-over-threshold } \\
\hline$Q_{d y}$ and $P_{3 d}$ & 0.1537 & 0.0606 & GUM & 1.0697 \\
\hline$Q_{d y}$ and $T_{3 d}$ & -0.0455 & -0.0077 & FRK & -0.0933 \\
\hline$Q_{d y}$ and $d S_{3 d}$ & 0.3828 & 0.4045 & FRK & 4.0447 \\
\hline \multicolumn{5}{|c|}{ S2 Yellowstone River at Corwin Springs, MT; M2 Multivariate Peak-over-threshold } \\
\hline$Q_{d y}$ and $P_{3 d}$ & 0.2381 & 0.1387 & GUM & 1.1793 \\
\hline$Q_{d y}$ and $T_{3 d}$ & 0.2311 & 0.1762 & CLT & 0.3145 \\
\hline$Q_{d y}$ and $d S_{3 d}$ & 0.4555 & 0.3112 & GAU & 0.4461 \\
\hline \multicolumn{5}{|c|}{ S2 Yellowstone River at Corwin Springs, MT; M3 Maximum Joint Empirical Probability } \\
\hline$Q_{d y}$ and $P_{3 d}$ & -0.1256 & -0.0906 & GAU & -0.1637 \\
\hline$Q_{d y}$ and $T_{3 d}$ & 0.2428 & 0.1351 & GUM & 1.1398 \\
\hline$Q_{d y}$ and $d S_{3 d}$ & 0.4287 & 0.2852 & GAU & 0.4247 \\
\hline \multicolumn{5}{|c|}{ S3 NF Clearwater River NR Canyon Ranger Station, ID; M2 Multivariate Peak-over-threshold } \\
\hline$Q_{d y}$ and $P_{3 d}$ & 0.1071 & 0.0673 & FRK & 0.6349 \\
\hline$Q_{d y}$ and $T_{3 d}$ & 0.0049 & 0.0026 & CLT & 0.0368 \\
\hline$Q_{d y}$ and $d S_{3 d}$ & 0.5273 & 0.4438 & GAU & 0.6124 \\
\hline
\end{tabular}

Note: All selected copula functions have one parameter.

\subsection{CONSTRUCTION OF JOINT DISTRIBUTIONS AND POTENTIAL APPLICATIONS}

After the selection of both marginal distributions and the copula function, a JCDF can be formed using Eq. (2.5). An example of the constructed bivariate JCDF for S2 using M2 events is shown in Figure 2-18. Because this copula-based approach allows for the combination of different types of marginal distributions and dependence structures, it provides great flexibility to derive the most suitable JCDF based on data.
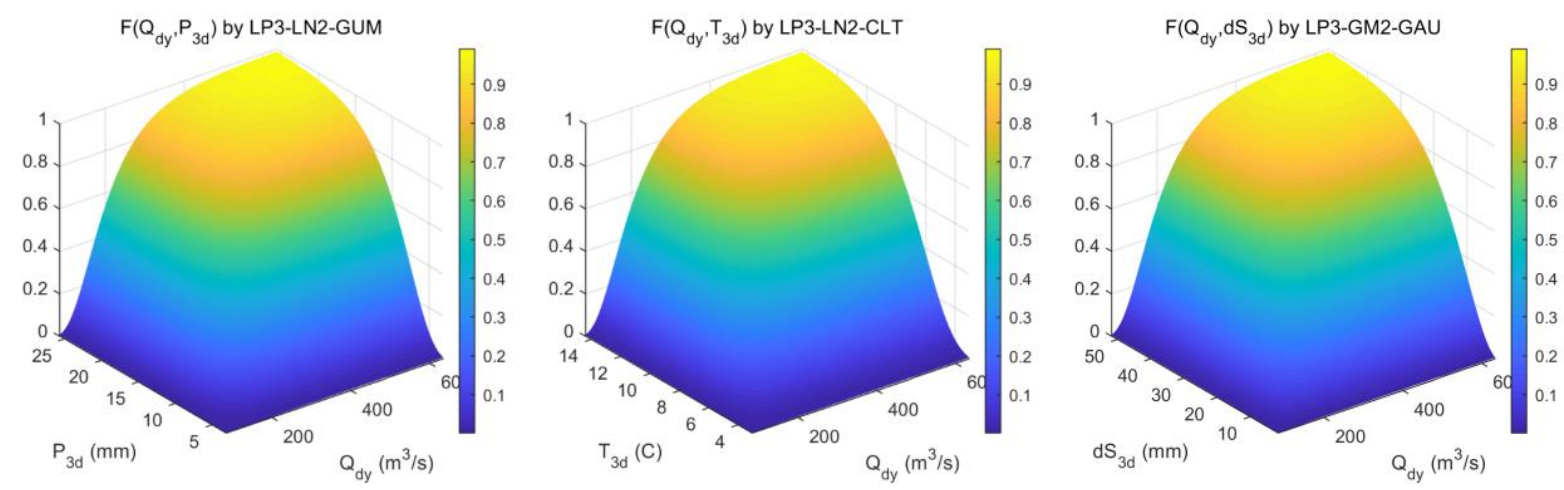

Figure 2-18. Example of constructed bivariate joint distributions by copulas for S2 using M2 events.

Additional examples are shown for each site in Figure 2-19 to Figure 2-22. In each figure, the constructed JCDFs are colored by different zones, in which the contours represent JCDF values from 0.1 to 0.9 in 0.1 increments. Contour lines derived from empirical copulas are also included for comparison. Overall, 
contour lines based on fitted copula are fairly similar to the ones based on empirical copulas, suggesting the reasonableness of the derived models.
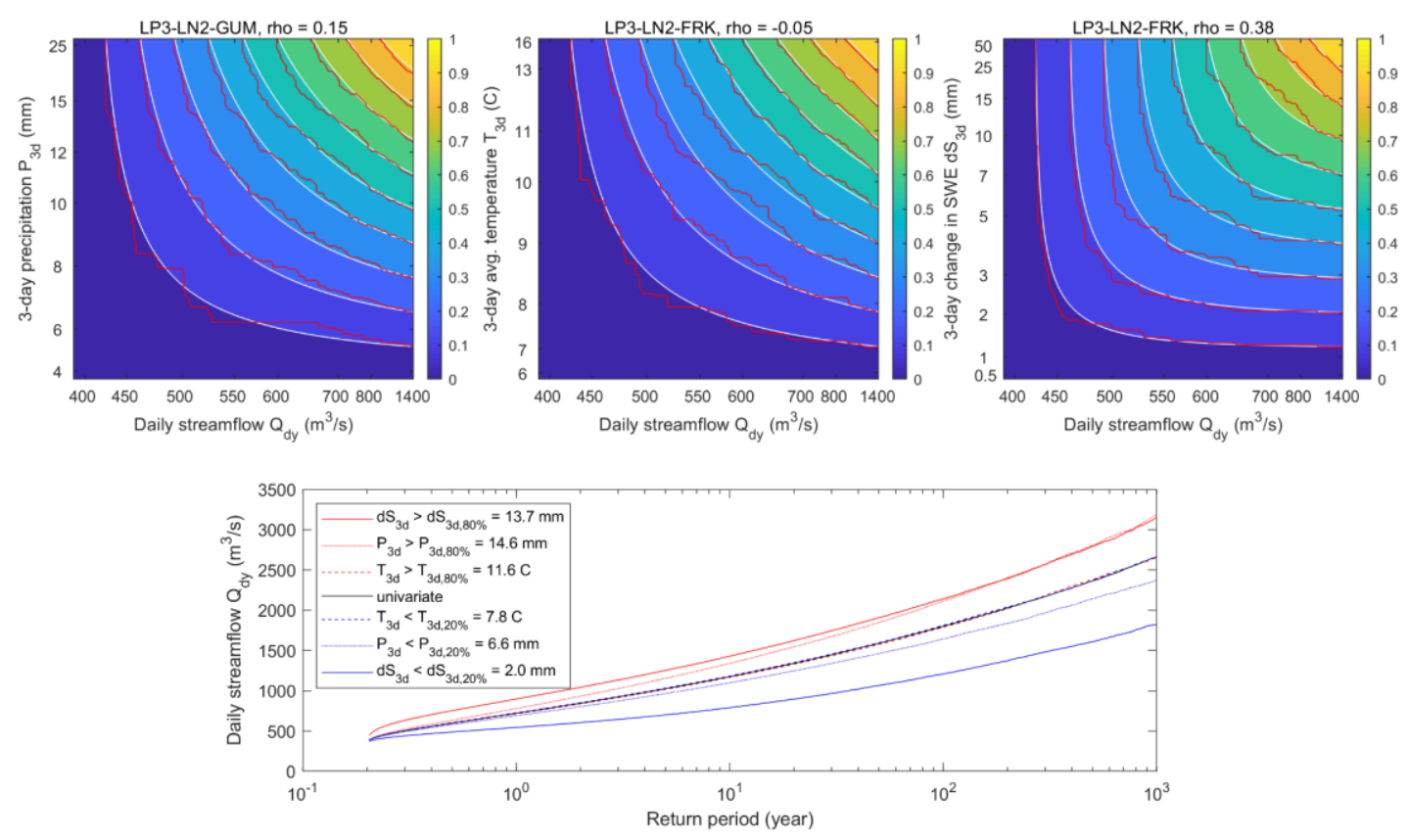

Figure 2-19. Joint distributions and conditional hazard curves of S1 using M2 events. The derived joint distributions were compared with the empirical copula values at each 0.1 contour line in the upper panels.

An example of the conditional hazard curves is shown in the lower panel.
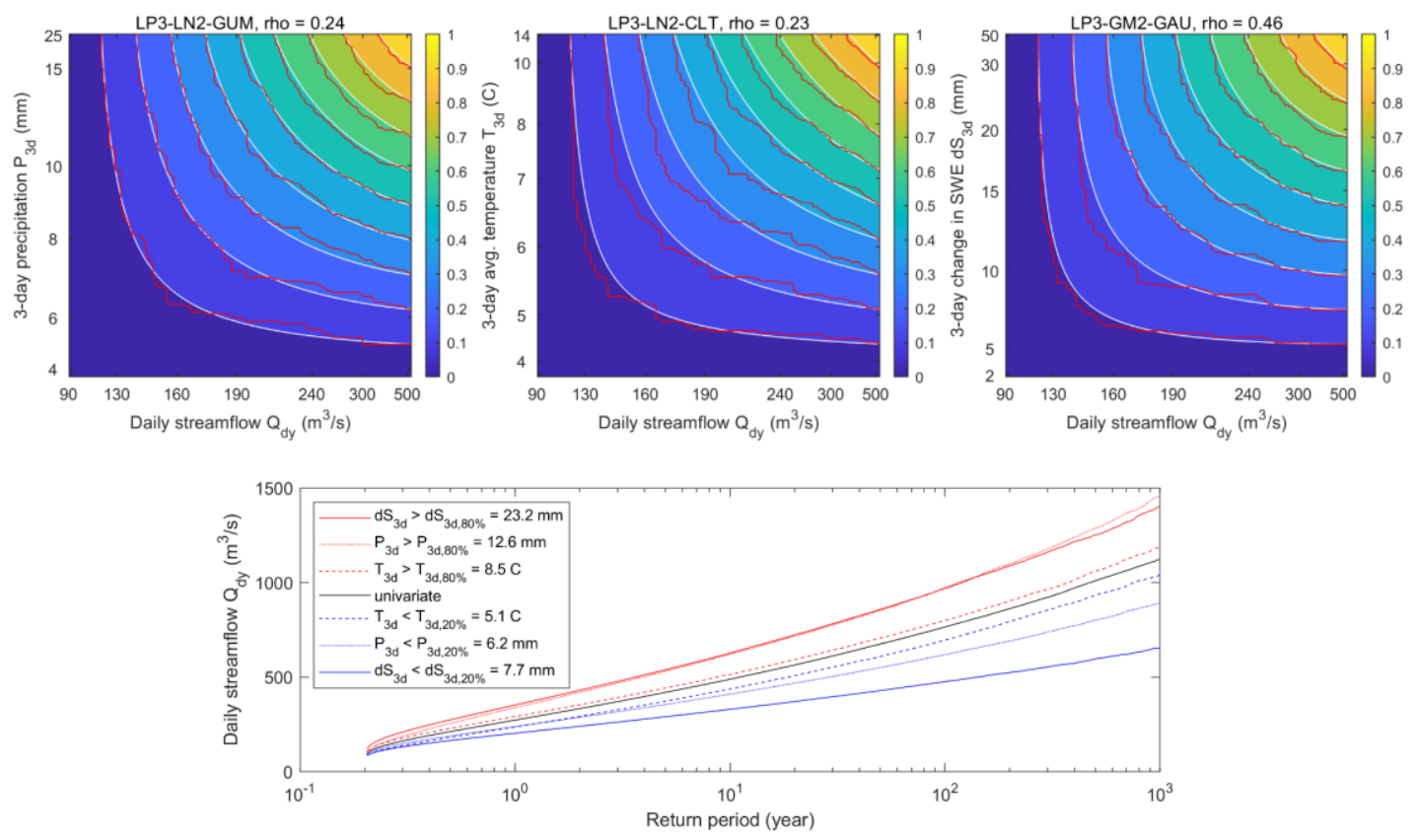

Figure 2-20. Joint distributions and conditional hazard curves of S2 using M2 events. The derived joint distributions were compared with the empirical copula values at each 0.1 contour line in the upper panels. An example of the conditional hazard curves is shown in the lower panel. 

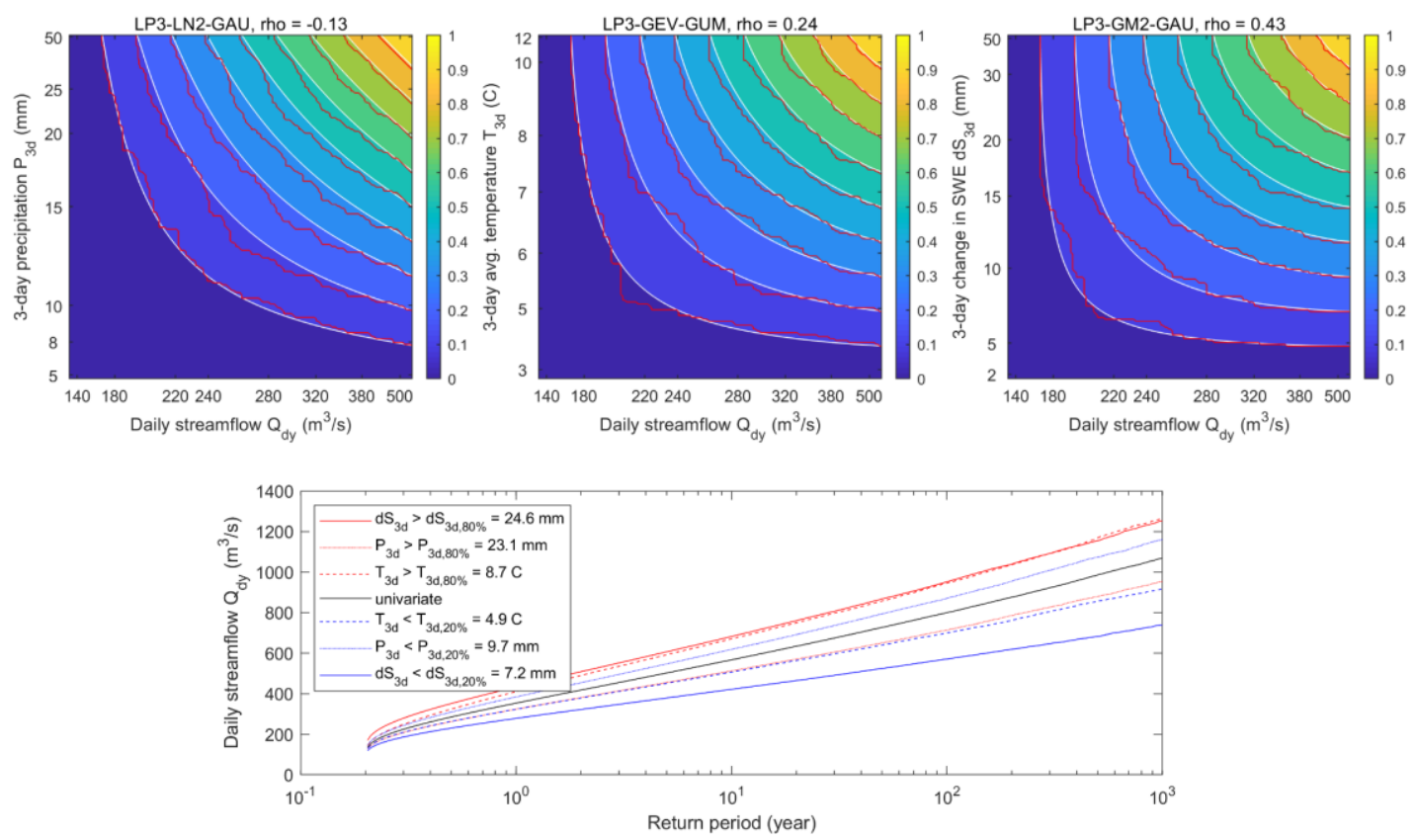

Figure 2-21. Joint distributions and conditional hazard curves of S2 using M3 events. The derived joint distributions were compared with the empirical copula values at each 0.1 contour line in the upper panels.

An example of the conditional hazard curves is shown in the lower panel.
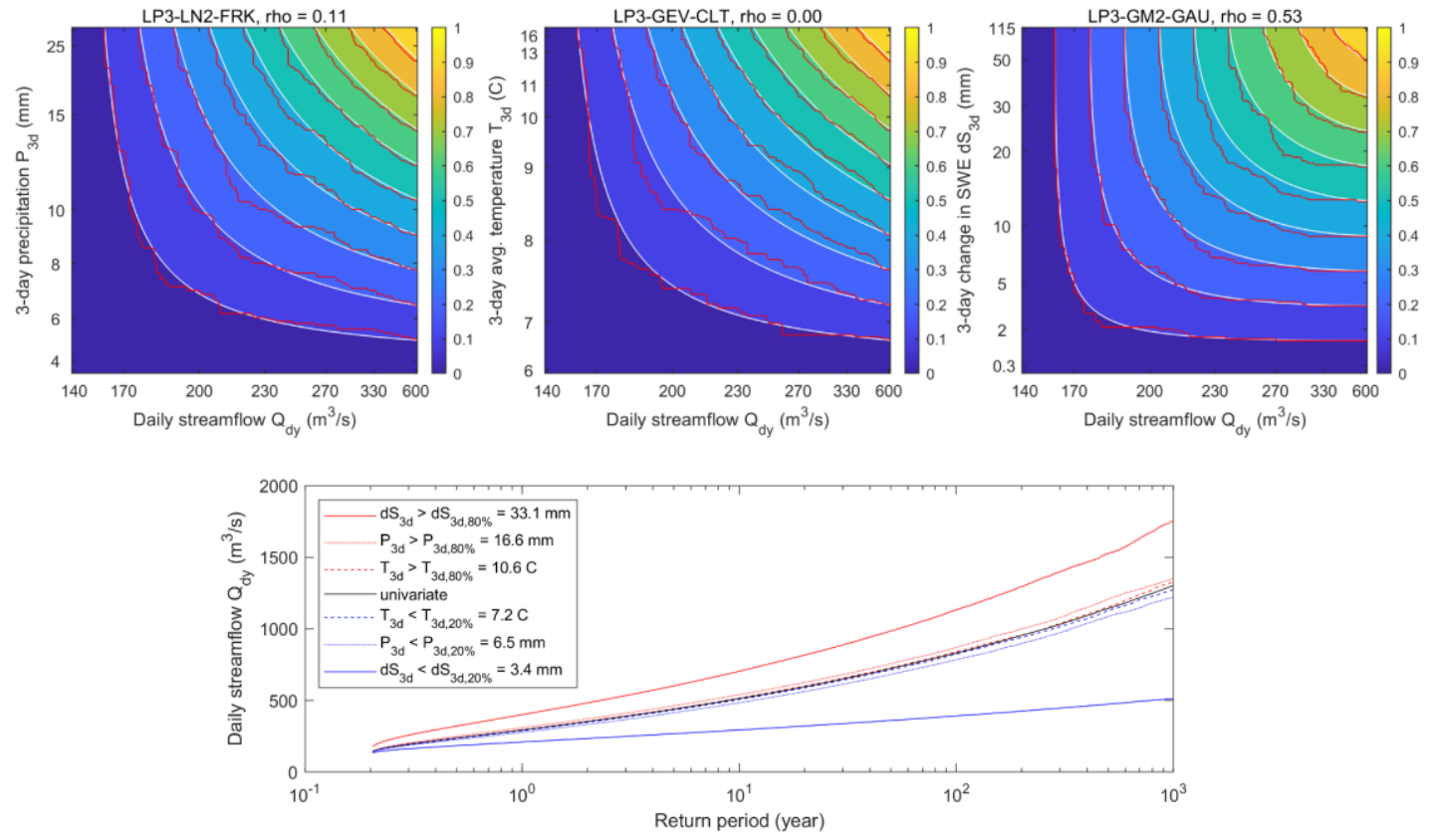

Figure 2-22. Joint distributions and conditional hazard curves of S3 using M2 events. The derived joint distributions were compared with the empirical copula values at each 0.1 contour line in the upper panels. An example of the conditional hazard curves is shown in the lower panel.

The derived JCDF allows a variety of applications. For instance, with a given condition of interest such as $\left\{d S_{3 d}>a\right\}$, the conditional distribution of $Q_{d y}$ can be written as 


$$
F_{Q_{d y}}\left(x \mid d S_{3 d}>a\right)=\frac{F_{Q_{d y}, d S_{3 d}}(x, a)}{1-F_{d S_{3 d}}(a)} .
$$

By plugging Eq. (2.10) into Eq. (2.4), the conditional hazard curves of $Q_{d y}$ were estimated. Seven hazard curves were plotted:

- Univariate: hazard curves derived by marginal distributions (from Section 2.5.1)

- $\quad\left\{P_{3 d}<P_{3 d, 20 \%}\right\}$ : conditional hazard curves when $P_{3 d}$ is less than the $20 \%$ quantile of $P_{3 d}$

- $\left\{T_{3 d}<T_{3 d, 20 \%}\right\}$ : conditional hazard curves when $T_{3 d}$ is less than the $20 \%$ quantile of $T_{3 d}$

- $\left\{d S_{3 d}<d S_{3 d, 20 \%}\right\}$ : conditional hazard curves when $d S_{3 d}$ is less than the $20 \%$ quantile of $d S_{3 d}$

- $\quad\left\{P_{3 d}>P_{3 d, 80 \%}\right\}$ : conditional hazard curves when $P_{3 d}$ is greater than the $80 \%$ quantile of $P_{3 d}$

- $\left\{T_{3 d}>T_{3 d, 80 \%}\right\}$ : conditional hazard curves when $T_{3 d}$ is greater than the $80 \%$ quantile of $T_{3 d}$

- $\left\{d S_{3 d}>d S_{3 d, 80 \%}\right\}$ : conditional hazard curves when $d S_{3 d}$ is greater than the $80 \%$ quantile of $d S_{3 d}$

The results show that a stronger correlation (such as between $Q_{d y}$ and $d S_{3 d}$ ) would lead to larger differences in conditional hazards, and vice versa. Therefore, the copula-based analysis can be applied if highly correlated variables can be identified. However, if the correlation between two variables is weak or statistically insignificant, one may not need to conduct multivariate analysis since the results will not be very different from the conventional univariate analysis. In the inland case study, the change of SWE had the highest correlation to streamflow and is the most important ancillary variable for the development of a multivariate distribution to analyze MMF and develop hazard curves.

\subsection{DISCUSSION}

Based on this inland case study, strengths and limitations of the copula-based MMF assessment approach were identified as described here.

- Compatibility with the conventional univariate frequency analysis: One important advantage of the copulas-based assessment approach is that it can build on the existing understanding of univariate frequency analysis. Many existing theories and tools can continue to be applied for analyzing marginal distributions. Additionally, similar to the selection of suitable PDFs to represent marginal distributions, a variety of copula functions can be tested and selected to represent dependence structure and form multivariate joint distributions. Although the concept of multivariate joint distribution may seem complicated, copulas can provide a natural extension to help better understand and leverage the existing univariate PFHA tools for multivariate applications.

- Flexibility with different types of distributions: Given the varying nature and physical processes, variables involved in MMF may follow different statistical distributions (e.g., LP3, GEV). Because the theory of copulas can allow for the separation of marginal distributions and dependence structures, the copula-based assessment is not limited to specific types of probabilistic distributions. This flexibility is desirable for wider applications of copulas in other MMF assessments.

- Definition of maximum events: The definition of maximum events represents one of the most significant differences to the conventional univariate frequency analysis. To support a meaningful statistical analysis, the selection of maximum events must consider their joint occurrence across all 
dimensions and cannot be done separately for each variable. To demonstrate its sensitivity in MMF assessments, three definitions of maximum events in this case study were compared, and relatively better performance was observed when using M2. However, because this issue has not been extensively studied, whether such a maximum event selection approach can also work for other applications is unclear. The most suitable strategy to select and define maximum events for the purpose of MMF assessment must be more fully explored.

- Data availability: Data availability is one of the greatest known challenges in univariate frequency analysis. Given the inconsistent data coverage, measurement, and accuracy across multiple variables, data availability is an even more critical issue for multivariate frequency analysis. Except for some data-rich watersheds and sites, the number of observations is likely insufficient for many potential MMF applications. Where data are sparse, one may need to identify suitable ways to leverage numeric model outputs to help expand the sample size for more reliable estimates. In other words, one will need to find a balance between the bias of numerical models and the bias caused by insufficient observations to support multivariate frequency analysis.

- Challenge of dimensionality: Although copulas have found wide applicability at the bivariate level, their application to higher dimensions $(\geq 3)$ is not straightforward and entails more mathematical challenges. For instance, the commonly used Archimedean copulas cannot be directly extended to higher dimensions, or they will lead to the same bivariate dependence structures of each pair of variables. At higher dimensions, meta-elliptical copulas (including Gaussian and t) or a mixture of bivariate copulas through the vine copulas approach (e.g., Vernieuwe et al. 2015) must be used. Data requirements will also increase significantly at higher dimensions, adding more challenges to the higher-dimension applications. 


\section{COASTAL MMF USE CASE STUDY}

\subsection{INTRODUCTION}

This case study used a Bayesian-motivated approach to assess MMF hazards in a coastal setting, specifically a site located in a tidal reach of the Delaware River. The flood forcing phenomena was a tropical cyclone (hurricane), and the flood mechanisms involved in the analysis included hurricaneinduced surge, precipitation, and river flow. The research objective was to develop a hazard curve for the river discharge flood severity metric accounting for the effects of river base flow, hurricane-induced surge, tides, and precipitation-induced runoff. This study aims to demonstrate the process of implementing the Bayesian-motivated approach to perform a probabilistic hazard assessment that accounts for MMFs. Although this is not a predictive modeling application, implementation of the Bayesian-motivated approach requires models that allow development of conditional probabilistic relationships between involved variables. To achieve this goal, this work combined a series of surrogate/statistical and analytical models to model river discharge caused by storm occurrence in a coastal area. These simplified models (relative to more robust numerical models) allow for demonstration of the Bayesian-motivated approach and are discussed in more detail in Section 3.4. This case study should be interpreted as a demonstration of a process rather than a definitive hazard assessment for the target location.

\subsection{COASTAL CASE STUDY SCOPE AND SETTING}

This case study focused on probabilistic assessment of MMF hazards from hurricane-induced storm surge, tides, and precipitation-induced discharge (rainfall runoff). The case study focused on a site located on the Delaware River near Trenton, New Jersey. Figure 3-1 shows the case study location.

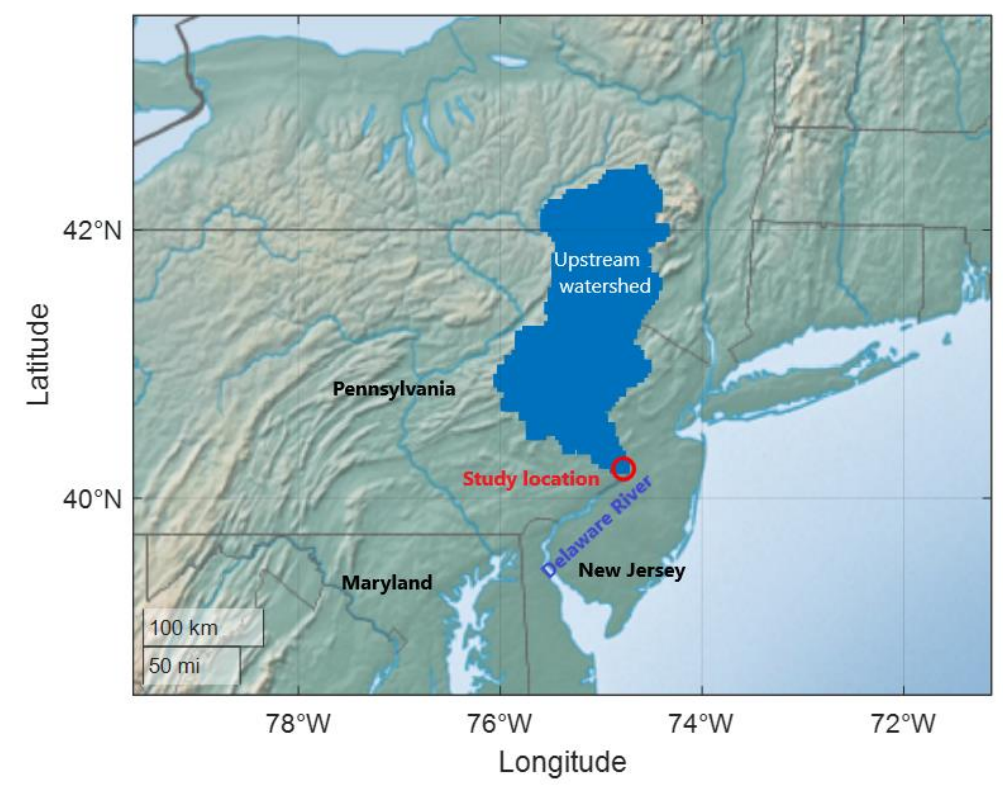

Figure 3-1. Case study location.

Multiple candidate case study regions were considered prior to the selection of the Trenton, New Jersey site. A key criterion used in selecting the case study location was the availability of the data needed to develop surrogate model components required to implement the framework described in Section 3.3. One 
of the limiting characteristics was the availability of 15-min stage-discharge measurements, which were not available along some tidally influenced rivers near other candidate locations.

The 15-min stage-discharge information was available near the case study location using USGS gage 01463500 (USGS 2021). Furthermore, Advanced Circulation (ADCIRC) model surge height simulations (i.e., simulations predicting storm surge still-water elevations as a function of hurricane characteristics) are readily available for the North Atlantic coast. Specifically, ADCIRC simulation results from the US Army Corps of Engineers (USACE) North Atlantic Comprehensive Coastal Study (NACCS) (NadalCaraballo et al. 2015) were obtained from the USACE Coastal Hazards System (USACE 2021). For this case study region, the furthest upstream location for which ADCIRC simulation data are available is NACCS save point 5373 . The save point is approximately $1.5 \mathrm{~km}$ downstream of USGS river gage 01463500. The location of save point 5373 was selected as the target study location for this case study.

To incorporate the effects of tides, NOAA tide gage 8539993 (NOAA 2021) was the closest gage to the study location. However, this tide gage is approximately $2.5 \mathrm{~km}$ downstream of the target study location (save point 5373). To confirm the consistency in the surge propagation between the locations of tide gage 8539993 and save point 5373, this study considered NACCS save point 7624, which is close to tide gage 8539993. The locations of the two save points, NOAA tide gage, and USGS gage are shown in Figure 3-2.
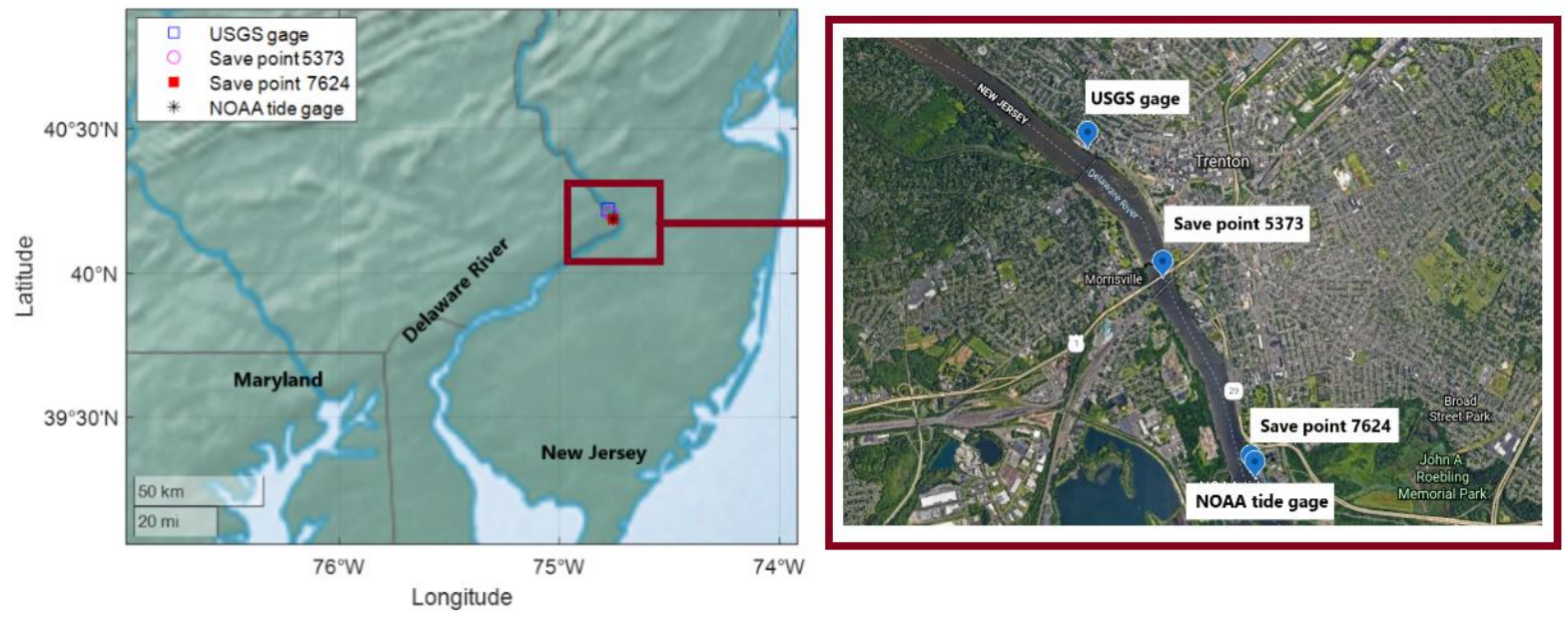

Figure 3-2. Location of the USGS gage, NOAA tide gage, save point 5373, and save point 7624.

\subsection{METHODOLOGY}

This case study used a Bayesian-motivated approach to generate a multi-mechanism probabilistic flood hazard curve using discharge as the flood severity parameter of interest. The Bayesian-motivated model developed in this case study is presented as a Bayesian network (BN; an acyclic graphical model). Because of its graphical nature, the BN provides a transparent means of presenting the proposed model and a convenient method for performing calculations involving the discretized forms of requisite integrals. However, the proposed approach does not require the use of BNs, and the fundamental integrals (specifically, their discrete counterparts) can be calculated directly.

The Bayesian-motivated approach in this case study began with understanding physical relationships between involved variables and using these relationships to develop the fundamental integrals that will be used to calculate exceedance probabilities necessary to develop hazard curves (Section 3.3). The next step 
involved defining storm parameters and developing models to predict response variables (Section 3.4) as a function of storm parameters and other intermediate quantities. After developing predictive models and the definition of storm parameters as model input, discretized values of input and predicted variables and their corresponding (conditional) probability mass functions (PMFs) were specified (Section 3.4.9). Using the PMFs, a hazard curve was generated (Section 3.5).

Figure 3-3 presents a BN formulation of the model used in this case study. Using the $\mathrm{BN}$ as a guide, the foundational integral for computing the probability that total river discharge $\left(Q_{\text {total }}\right)$ exceeds flood discharge severity $q$ is represented as

$$
\begin{aligned}
& P\left(Q_{\text {total }}>q\right)=\int_{-\infty}^{\infty} \ldots \int_{-\infty}^{\infty} P\left(Q_{\text {total }}>q \mid Q_{\{\eta, R, T\}}, Q_{P}\right) f\left(Q_{P} \mid \widehat{Q}_{P}, \varepsilon_{Q_{P}}\right) f\left(\varepsilon_{Q_{P}}\right) f\left(\widehat{Q}_{P} \mid P_{B A}\right) \\
& f\left(P_{B A} \mid \hat{P}_{B A}, \varepsilon_{P_{B A}}\right) f\left(\varepsilon_{P_{B A}}\right) f\left(\hat{P}_{B A} \mid V_{w}, \theta, x_{0}, V_{f}\right) f\left(V_{w} \mid \widehat{V}_{w}, \varepsilon_{V_{w}}\right) f\left(\varepsilon_{V_{w}}\right) f\left(\widehat{V_{w}} \mid \Delta p\right) f(\Delta p) f\left(x_{0}\right) \\
& f\left(V_{f}\right) f(\theta) f\left(R_{\max }\right) f\left(Q_{\{\eta, R, T\}} \mid \hat{Q}_{\{\eta, R, T\}}, \varepsilon_{Q_{\{\eta, R, T\}}}\right) f\left(\varepsilon_{Q_{\{\eta, R, T\}}}\right) f\left(\hat{Q}_{\{\eta, R, T\}} \mid \eta, Q_{R}, T\right) f\left(Q_{R}\right) \\
& f(T) f\left(\eta \mid \hat{\eta}, \varepsilon_{\eta}\right) f\left(\varepsilon_{\eta}\right) f\left(\hat{\eta} \mid R_{\max }, \theta, x_{0}, V_{f}, \Delta p\right) d \Omega,
\end{aligned}
$$

where

- $Q_{\text {total }}$ : total discharge, accounting for hurricane-induced surge, precipitation-induced runoff, concurrent base flow, and tides (cfs),

- $Q_{R}$ : concurrent river base flow (cfs),

- $Q_{P}$ : hurricane-induced precipitation discharge (cfs),

- $Q_{\{\eta, R, T\}}$ : discharge caused by surge, river base flow, and tides (cfs),

- $T$ : elevation of the tides ( $\mathrm{ft})$,

- $P_{B A}$ : average basin precipitation (in./day),

- $\quad \eta$ : surge elevation $(\mathrm{m})$,

- $\quad \Delta p$ : the storm's central pressure deficit $(\mathrm{hPa})$, computed as the difference between a peripheral atmospheric pressure of $1,013 \mathrm{hPa}$ and the storm's central pressure $(\mathrm{hPa})$,

- $\quad V_{f}$ : the storm's forward velocity (speed) $(\mathrm{km} / \mathrm{h})$,

- $R_{\max }$ : the storm's radius to the maximum wind $(\mathrm{km})$,

- $\theta$ : the storms heading (direction) measured in degrees clockwise from north,

- $\quad x_{0}$ : the storm's reference location (e.g., landfall location), and

- $V_{w}$ : wind velocity $(\mathrm{km} / \mathrm{h})$. 


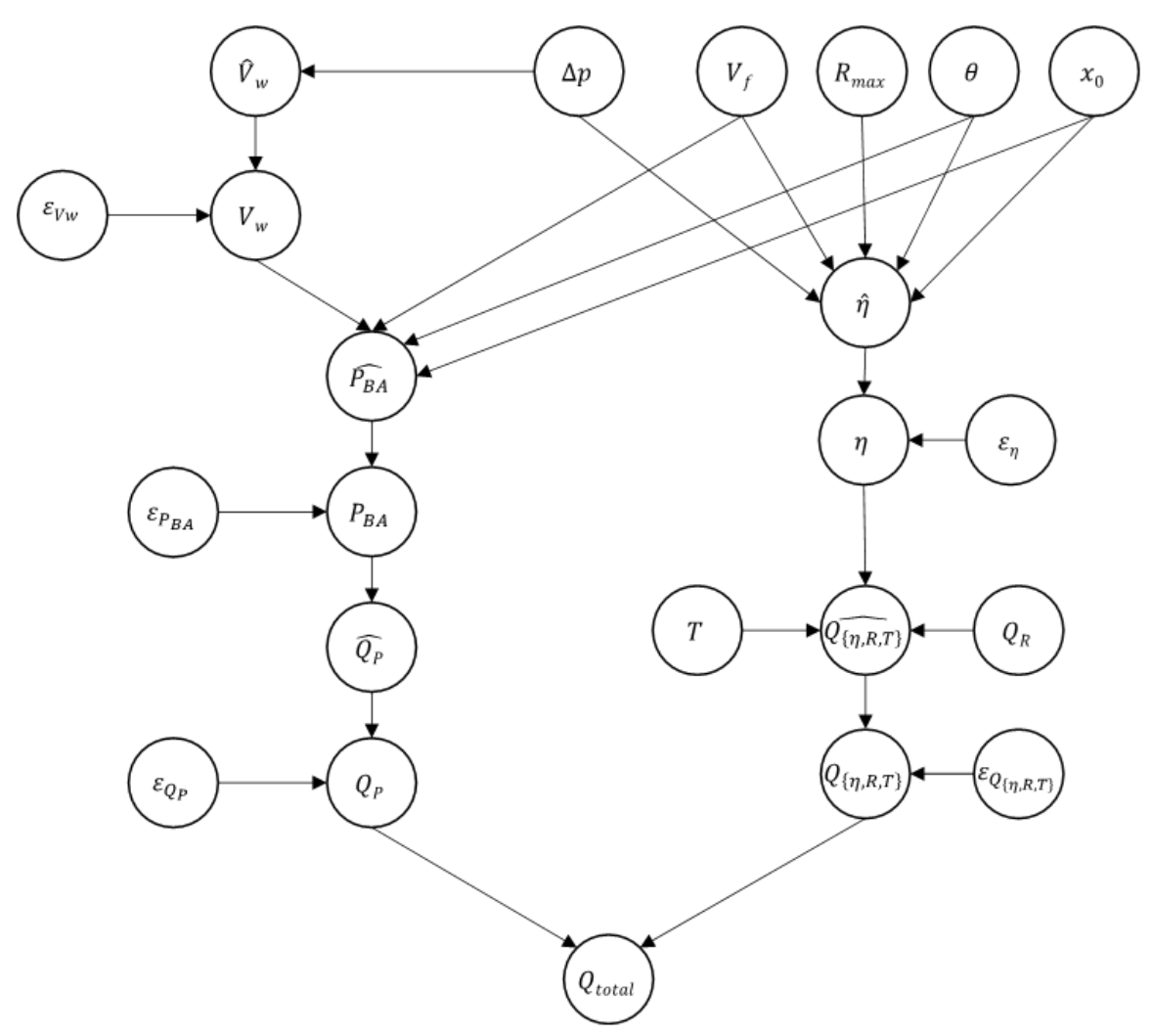

Figure 3-3. BN representation of fundamental integral used in case study.

The "hat" notation indicates quantities predicted by a model. The " $\varepsilon$ quantities" refer to model errors with subscripts, indicating the model to which they apply.

The integral in Eq. (3.1) is calculated in discrete form as

$$
\begin{aligned}
& P\left(Q_{\text {total }}>q\right) \\
& =\sum \ldots \sum P\left(Q_{\text {total }}>q \mid Q_{\{\eta, R, T\}}, Q_{P}\right) p\left(Q_{P} \mid \hat{Q}_{P}, \varepsilon_{Q_{P}}\right) p\left(\varepsilon_{Q_{P}}\right) p\left(\hat{Q}_{P} \mid P_{B A}\right) \\
& p\left(P_{B A} \mid \hat{P}_{B A}, \varepsilon_{P_{B A}}\right) p\left(\varepsilon_{P_{B A}}\right) p\left(\hat{P}_{B A} \mid V_{w}, \theta, x_{0}, V_{f}\right) p\left(V_{w} \mid \widehat{V}_{w}, \varepsilon_{V_{w}}\right) p\left(\varepsilon_{V_{w}}\right) p\left(\widehat{V_{w}} \mid \Delta p\right) p(\Delta p) p\left(x_{0}\right) \\
& p\left(V_{f}\right) p(\theta) p\left(R_{\text {max }}\right) p\left(Q_{\{\eta, R, T\}} \mid \hat{Q}_{\{\eta, R, T\}}, \varepsilon_{Q_{\{\eta, R, T\}}}\right) p\left(\varepsilon_{Q_{\{\eta, R, T\}}}\right) p\left(\hat{Q}_{\{\eta, R, T\}} \mid \eta, Q_{R}, T\right) \\
& p\left(Q_{R}\right) p(T) p\left(\eta \mid \hat{\eta}, \varepsilon_{\eta}\right) p\left(\varepsilon_{\eta}\right) p\left(\hat{\eta} \mid R_{\max }, \theta, x_{0}, V_{f}, \Delta p\right) .
\end{aligned}
$$

Using a Poisson process assumption regarding the occurrence of hurricane events (as is typically assumed), the annual rate of exceedance is computed as

$$
\lambda_{Q_{\text {total }>q}}=\lambda_{H} * P\left(Q_{\text {total }}>q\right)
$$

where $\lambda_{H}$ is the rate at which hurricanes affect an area, and $P\left(Q_{t o t a l}>q\right)$ is computed as in Eq. (3.2). 


\subsection{KEY ASSUMPTIONS}

After developing the quantitative expression for the probability of exceedance of the severity measure, the next step in the analysis is to calculate the conditional and marginal probability distributions required to evaluate the expression shown in Eq. (3.2). The development of these distributions requires evaluation of statistical data and use of the predictive models. Five key predictive models were used in this case study. These models are shown in Figure 3-4:

- Surge model: a surrogate model for predicting surge height $(\eta)$ as a function of hurricane parameters $\left(R_{\max }, X_{o}, V_{f}, \theta, \Delta p\right)$

- Wind model: a statistical model for predicting maximum wind velocity $\left(V_{w}\right)$ as a function of central pressure deficit $(\Delta p)$

- Precipitation model: a statistical-empirical model for predicting hurricane-induced precipitation across the regional watershed as a function of selected hurricane characteristics (i.e., hurricane track and a decayed wind velocity)

- Precipitation-induced discharge model: a statistical model for predicting precipitation-induced discharge

- Surge-, tide-, and river base flow-induced discharge model (also referred to herein as the combined discharge model): a statistical model for predicting river discharge caused by surge, tides, and river base flow

These models were used to develop the conditional probability tables (CPTs) associated with the nodes highlighted in the BN in Figure 3-5. Additional information about the development of conditional probability distributions is provided in the subsections that follow (Section 3.4.1 through Section 3.4.9). Once all distributions were defined, the next step in the analysis involved discretization of all variables (Section 3.4.9) and the evaluation of the expression in Eq. (3.2). 


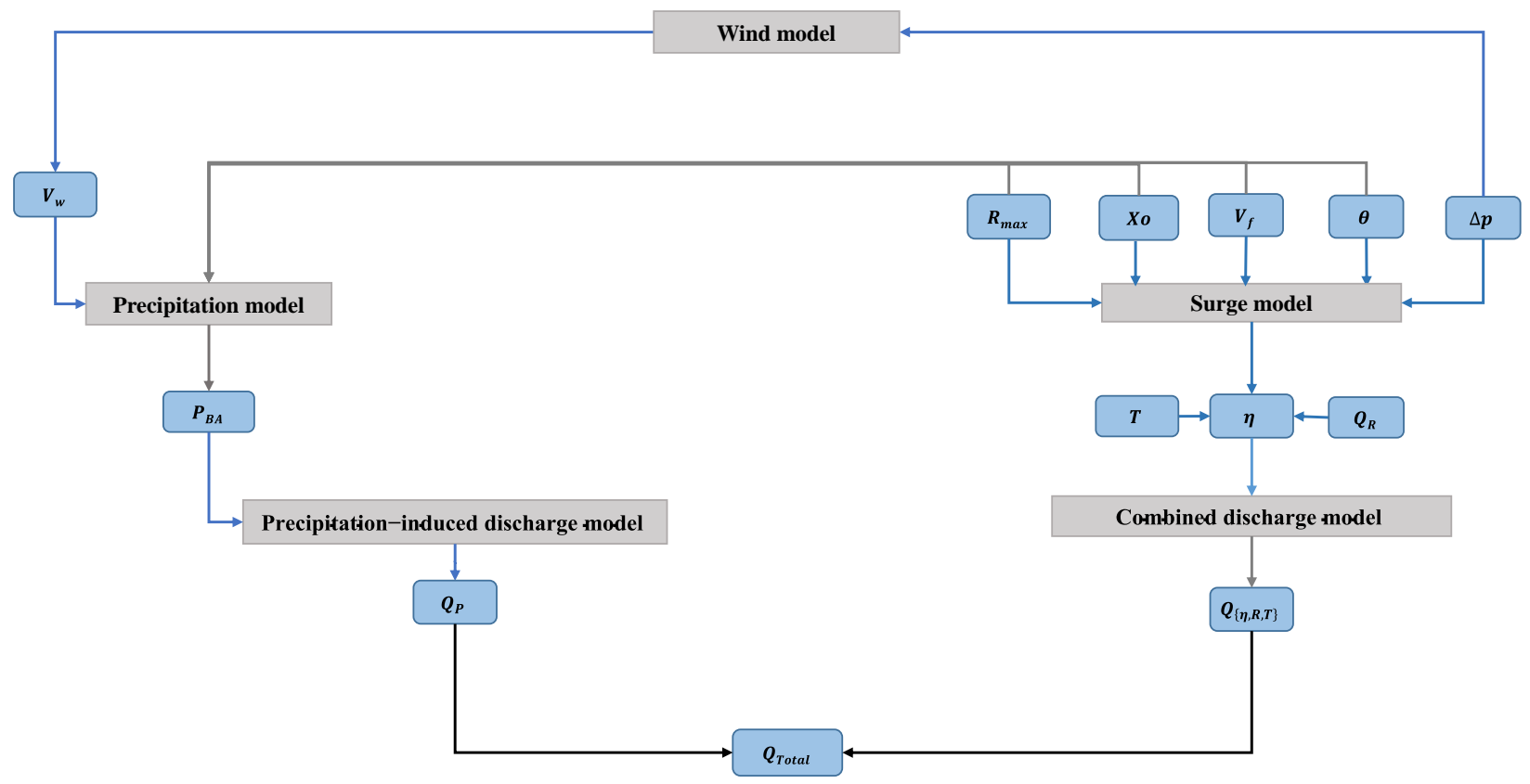

Figure 3-4. Key models used in the case study.

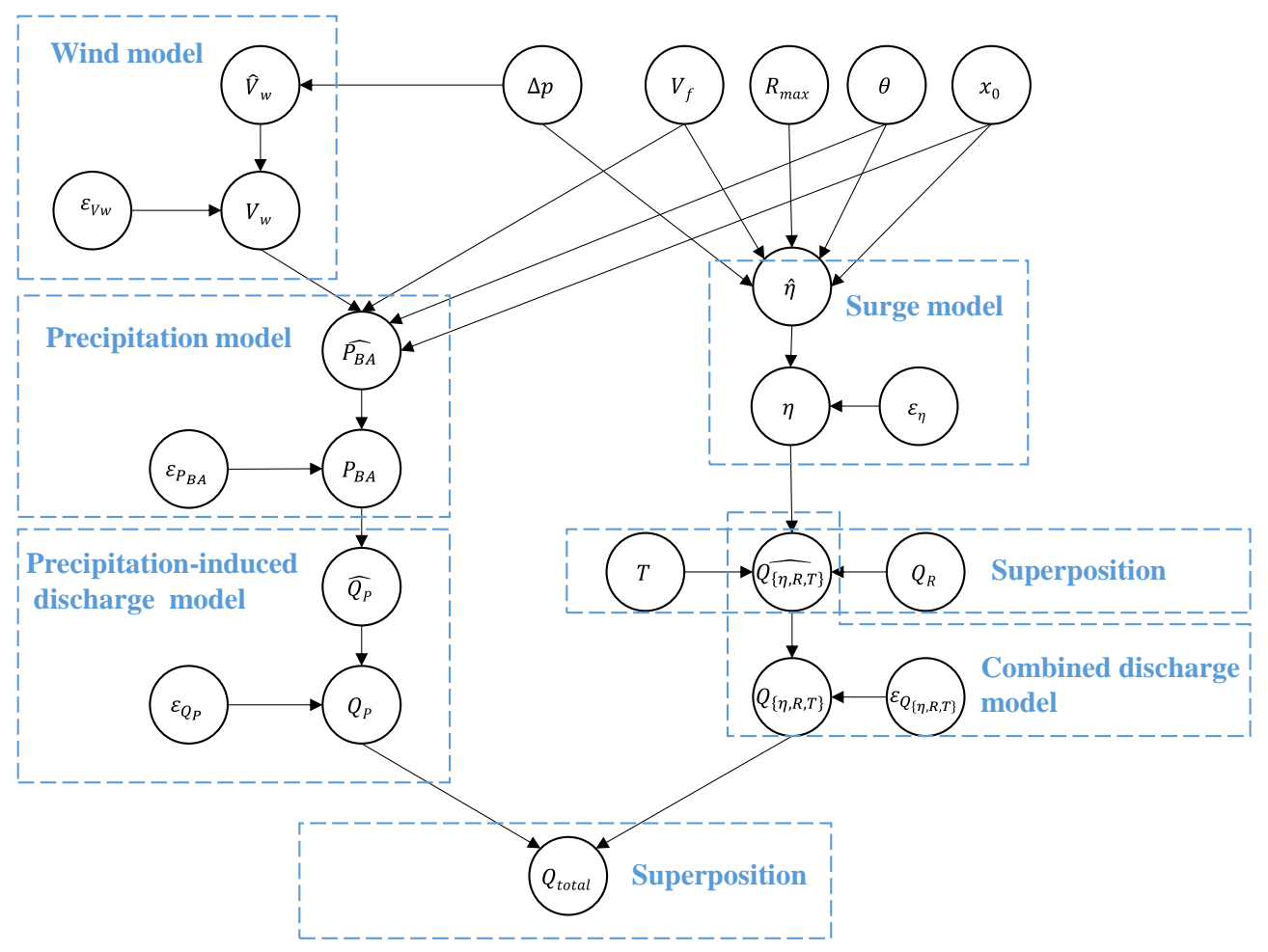

Figure 3-5. Key models used to develop BN representation used in the case study.

\subsubsection{Storm parameters}

In this case study, a hurricane was parameterized using five quantities: 
- $\Delta p=$ the storm's central pressure deficit $(\mathrm{hPa})$, computed as the difference between a peripheral atmospheric pressure of $1,013 \mathrm{hPa}$ and the storm's central pressure $(\mathrm{hPa})$,

- $V_{f}=$ the storm's forward velocity $($ speed $)(\mathrm{km} / \mathrm{h})$,

- $\quad R_{\max }=$ the storm's radius to the maximum wind $(\mathrm{km})$,

- $\theta=$ the storm's heading (direction) measured in degrees clockwise from north, and

- $\quad x_{0}=$ the storm's reference location (e.g., landfall location).

In the BN in Figure 3-3, these quantities are represented by the root (top) nodes.

Typically, the development of probability distributions for storm parameters requires statistical assessment of historical hurricane track data (potentially augmented with synthetic data sources). In this study, the distributions suggested by NACCS for the area under study (Nadal-Caraballo et al. 2015) were used to define the distributions assigned to storm parameters: distributions $f(\Delta p), f\left(x_{0}\right), f\left(V_{f}\right), f(\theta)$, and $f\left(R_{\max }\right)$ in Eq. (3.2). Consistent with NACCS, hurricane parameters were treated as statistically independent quantities. Table 3-1 shows the distributions and corresponding parameters. NACCS considered three sub-regions for the analysis in the North Atlantic region. Distributions and corresponding parameters in Table 3-1 are related to Region 2, where the area under study is located.

Table 3-1. Distributions and corresponding parameters for hurricane parameters.

\begin{tabular}{|c|c|c|c|c|}
\hline No. & $\begin{array}{l}\text { Hurricane } \\
\text { parameters }\end{array}$ & Distribution & Functional form & $\begin{array}{c}\text { Distribution } \\
\text { parameters }\end{array}$ \\
\hline 1 & $\Delta p$ & $\begin{array}{l}\text { Doubly truncated Weibull } \\
\text { distribution }\end{array}$ & $\begin{array}{l}P[\Delta p>x] \\
=\frac{\exp \left[-\left(\frac{x}{U}\right)^{k}\right]-\exp \left[-\left(\frac{\Delta p_{2}}{U}\right)^{k}\right]}{\exp \left[-\left(\frac{\Delta p_{1}}{U}\right)^{k}\right]-\exp \left[-\left(\frac{\Delta p_{2}}{U}\right)^{k}\right]}\end{array}$ & $\begin{array}{l}\Delta p_{1}=25 \mathrm{hpa} \\
\Delta p_{2}=93 \mathrm{hpa} \\
U=35.77 \\
k=1.41\end{array}$ \\
\hline 2 & $R_{\max }$ & LN distribution & $f(x)=\frac{1}{x \zeta \sqrt{2 \pi}} \exp \left[-\frac{1}{2}\left(\frac{\ln (x)-\lambda}{\zeta}\right)^{2}\right]$ & $\lambda=4.215, \zeta=0.45$ \\
\hline 3 & $V_{f}$ & NOR distribution & $f(x)=\frac{1}{\sigma \sqrt{2 \pi}} \exp \left[-\frac{1}{2}\left(\frac{x-\mu}{\sigma}\right)^{2}\right]$ & $\mu=44.05, \sigma=16.06$ \\
\hline 4 & $\theta$ & NOR distribution & $f(x)=\frac{1}{\sigma \sqrt{2 \pi}} \exp \left[-\frac{1}{2}\left(\frac{x-\mu}{\sigma}\right)^{2}\right]$ & $\mu=16.48, \sigma=36.17$ \\
\hline 5 & $x_{0}$ & Uniform distribution & - & - \\
\hline
\end{tabular}

\subsubsection{Storm surge model}

The storm surge model represents surge height $(\eta)$ as a function of a surge prediction $(\hat{\eta})$ and a prediction error term $\left(\varepsilon_{\eta}\right)$ :

$$
\eta=\hat{\eta}+\varepsilon_{\eta}
$$


This function is used to generate the conditional distribution $p\left(\eta \mid \hat{\eta}, \varepsilon_{\eta}\right)$ shown in Eq. (3.2) (and equivalently, the CPT assigned to node $\eta$ in Figure 3-3). Conceptually, Eq. (3.4) specifies that for each combination of $\hat{\eta}$ and $\varepsilon_{\eta}, \eta$ is simply a deterministic combination of both quantities. However, when $\hat{\eta}$ and $\varepsilon_{\eta}$ are discretized, a finite number of combinations of the discretized "bins" are associated with $\hat{\eta}$ and $\varepsilon_{\eta}$. Letting $\widehat{\eta}_{l}$ indicate $\left\{\eta_{i} \leq \hat{\eta}<\eta_{i+1}\right\}$ and $\varepsilon_{\eta, j}$ indicate $\left\{\varepsilon_{\eta, j} \leq \varepsilon_{\eta}<\varepsilon_{\eta, j+1}\right\}$, then $p\left(\eta \mid \widehat{\eta}_{l}, \varepsilon_{\eta, j}\right)$ represents the conditional PMF of $\eta$ given that $\hat{\eta}$ and $\varepsilon_{\eta}$ are within the ranges $\left\{\eta_{i} \leq \hat{\eta}<\eta_{i+1}\right\}$ and $\left\{\varepsilon_{\eta, j} \leq \varepsilon_{\eta}<\varepsilon_{\eta, j+1}\right\}$, respectively. To limit the impact of this discretization, this conditional PMF was developed using Monte Carlo simulation, as described in Section 3.4.9. This approach was used to develop the conditional PMFs associated with all deterministic functions presented in this case study.

The conditional distribution $p\left(\hat{\eta} \mid R_{\max }, \theta, x_{0}, V_{f}, \Delta p\right)$ in Eq. (3.2) (and equivalently, the CPT for node $\hat{\eta}$ in Figure 3-3) is generated using a surrogate model developed to predict surge height ( $\hat{\eta}, \mathrm{m}$ MSL) as a function of representative synthetic hurricane track parameters:

$$
\hat{\eta}=g\left(\Delta p, V_{f}, \theta, R_{\max }, x_{0}\right)
$$

where all quantities are as defined in Section 3.3.

In this use case study, a surrogate model using a Gaussian process regression (GPR) (Al Kajbaf and Bensi 2020; Bass and Bedient 2018; Jia et al. 2016; Jia and Taflanidis 2013) was developed. The regression model was trained to emulate the ADCIRC-computed surge values generated by NACCS (NadalCaraballo et al. 2015) for the closest study save point to the target location (save point 5373; see Figure 3-1). The data required for training and testing the model were obtained from the USACE coastal hazard system website (https://chswebtool.erdc.dren.mil/). Alternative predictive models, including neural network (NNET) and support vector machine (SVM), were considered and tested; however, the GPR model showed the overall best performance. For example, Figure 3-6 shows the surge height predicted using these surrogate models versus the simulated ADCIRC surge height for one example of a 70/30 holdout validation (i.e., 70\% of the data are designated as training data and used to "fit" the model, and the remaining $30 \%$ of the data are withheld for testing the "fitted model" against "unseen" data). Table 3-2 shows the estimated root mean square error (RMSE) and correlation for these three models for this single holdout validation example. Given the better performance of the GPR for the proposed application and the commonality of its use in surge surrogate models, the GPR was used for this study. 


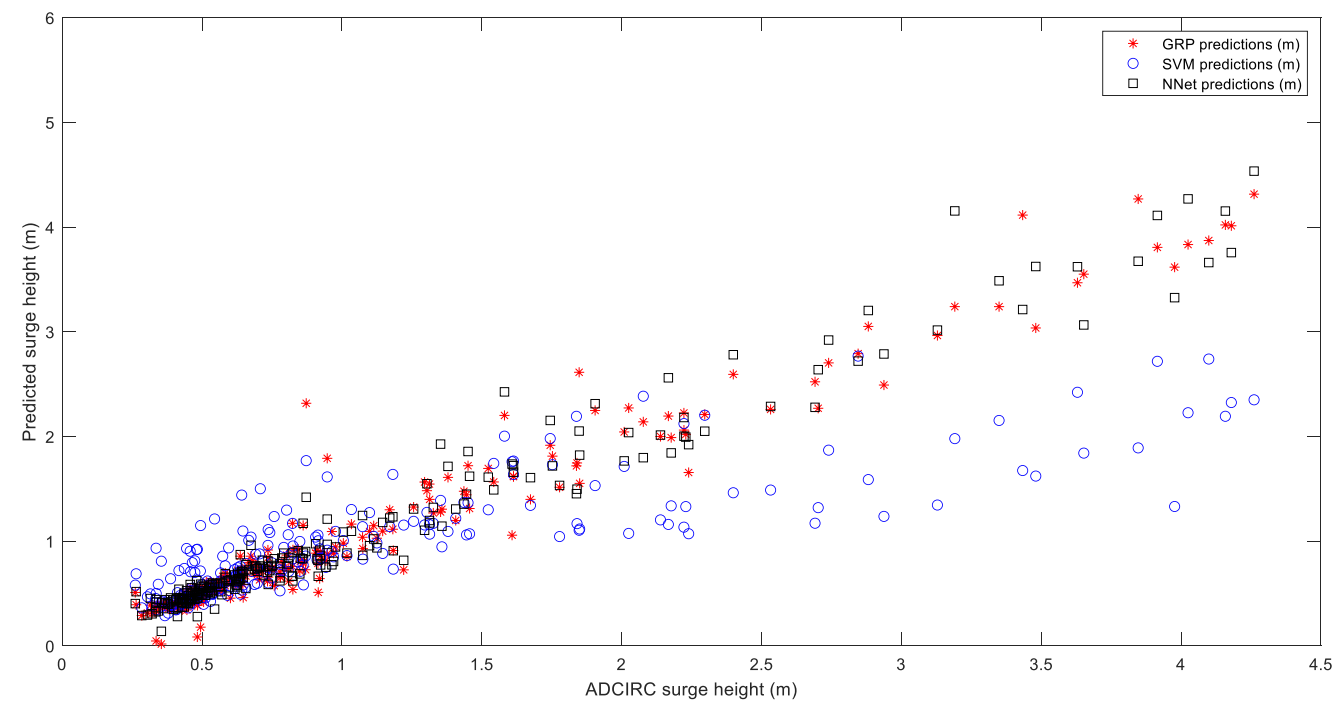

Figure 3-6. Correlation between ADCIRC-simulated surge height and predicted surge height using GPR, NNET, and SVM models.

Table 3-2. Correlation and RMSE values related to GPR, NNET, and SVM models.

\begin{tabular}{|l|c|c|c|}
\hline \multicolumn{1}{|c|}{ Error metrics } & GPR & NNET & SVM \\
\hline $\begin{array}{l}\text { Correlation coefficient } \\
\text { (surrogate versus ADCIRC-simulated surge) }\end{array}$ & 0.98 & 0.97 & 0.83 \\
\hline RMSE (m) & 0.20 & 0.22 & 0.56 \\
\hline
\end{tabular}

To provide insights regarding the magnitude of the NACCS ADCIRC-computed surge at the save point, Figure 3-7 shows the peak surge height estimated for each of the 1,031 storms in the synthetic tropical cyclone storm suite considered in NACCS. Surge was computed for base conditions (i.e., storm surge computed on mean sea level without tidal or sea-level rise considerations).

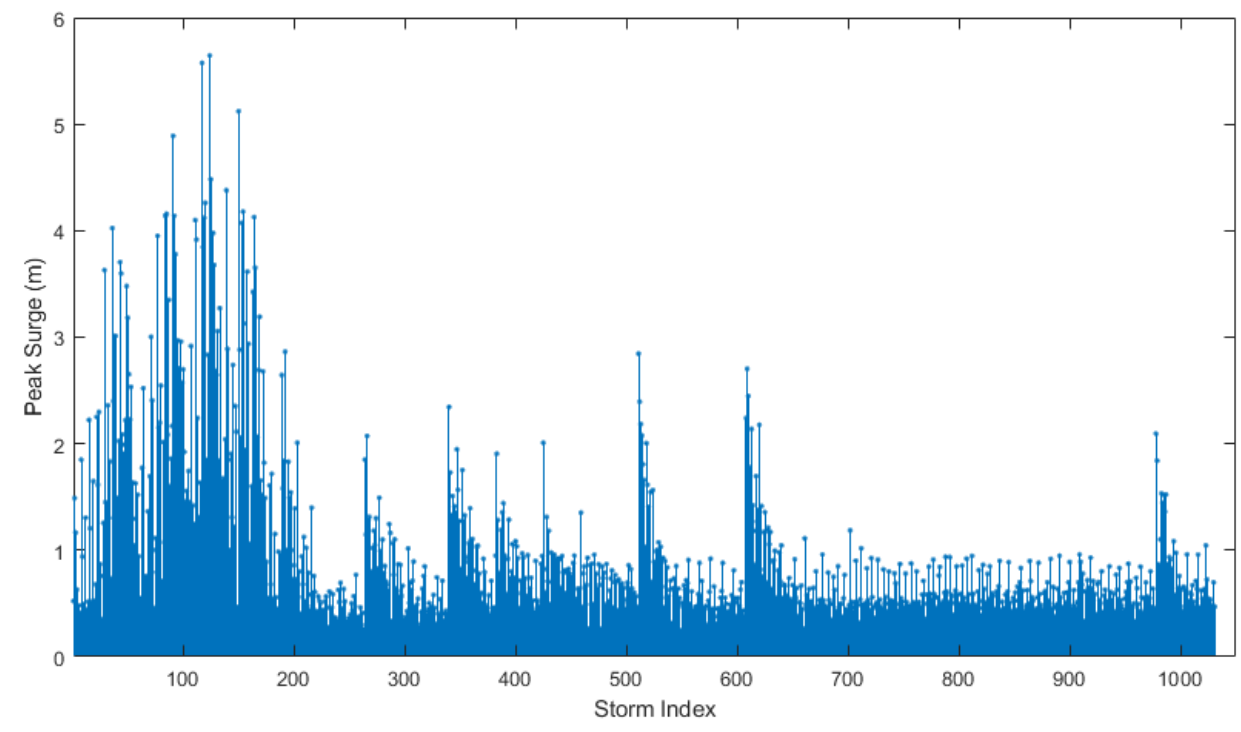

Figure 3-7. NACCS ADCIRC-computed storm surge at the target save point for 1,031 storms. 
To assess the out-of-sample prediction abilities of the GPR model used to estimate peak storm surge, a holdout validation was performed using 50 holdout folds, each consisting of a 70/30 split of randomly selected training and testing (holdout) data. For each fold, $70 \%$ of the data were designated as training data, which was used to fit the model. The remaining $30 \%$ of the data were withheld for testing the fitted model against unseen data. Figure 3-8 (left) shows a scatterplot of surrogate model and ADCIRC simulations for the out-of-sample predictions across all 50 holdout sets. Figure 3-8 (right) shows a histogram of the computed correlation coefficient across the 50 folds. The correlation coefficients vary from approximately 0.96 to 0.99 .
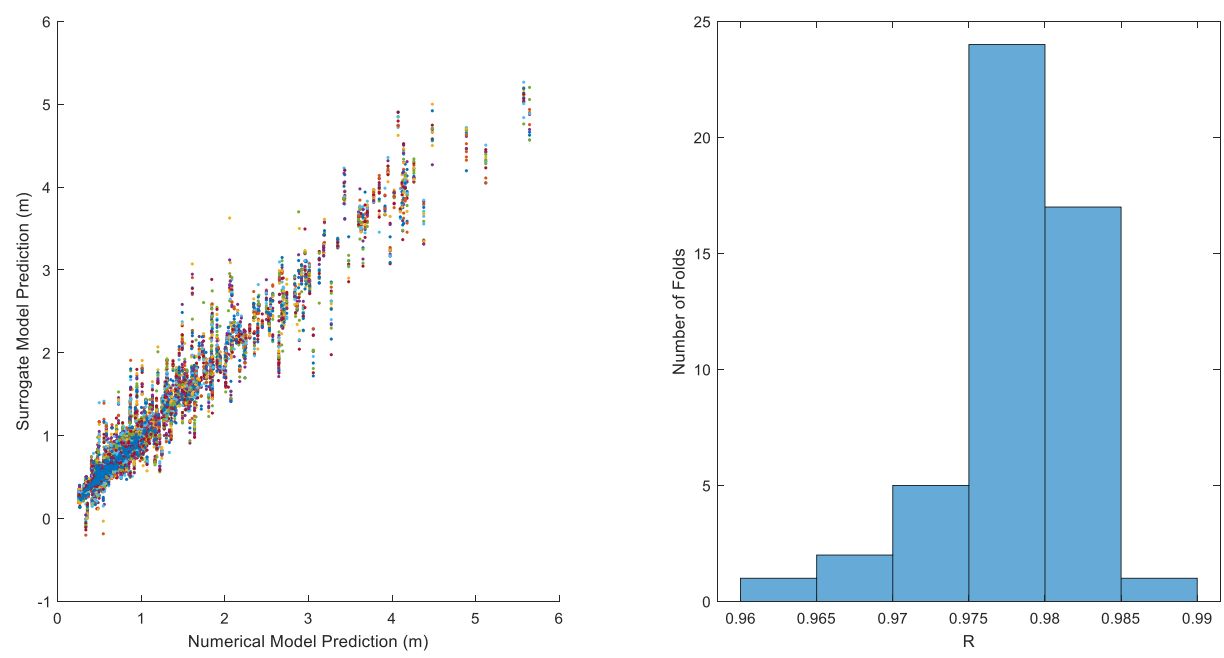

Figure 3-8. (left) Scatterplot of surrogate and numerical (ADCIRC) model predictions related to surge height (m) for the out-of-sample predictions for the $\mathbf{5 0}$ holdout sets, and (right) histogram of computed correlation coefficients across the 50 holdout sets.

Figure 3-9 (left) presents a scatterplot of computed out-of-sample prediction errors (ADCIRC estimate minus surrogate model prediction), and Figure 3-9 (right) shows the histogram of the computed RMSE across the 50 folds. The RMSE varied between 0.14 and $0.22 \mathrm{~m}$ across the 50 folds, and the mean RMSE was $0.16 \mathrm{~m}$. The mean RMSE was used in characterizing the distribution of the model error term used in the analysis. The overall bias (mean error) was small (ranging between -0.02 and $0.02 \mathrm{~m}$ ). However, a trend is observable in Figure 3-9 (left), with larger ADCIRC-estimated surges being associated with a larger (positive) error, suggesting the surrogate model may under-predict larger surge values. Although not addressed in this case study, modification of the standard deviation of the error term in the BN model to account for larger errors with increasing surge height is identified as an area for future study. 

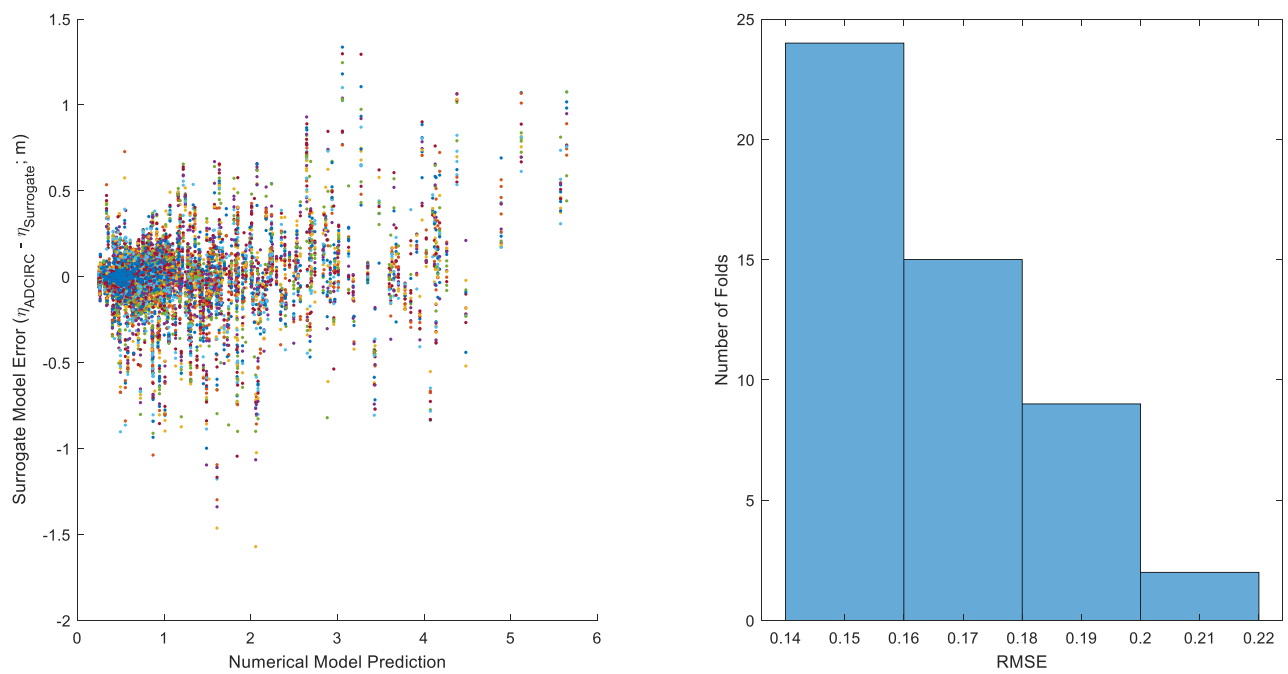

Figure 3-9. (left) Scatterplot of computed out-of-sample prediction error and numerical (ADCIRC) model predictions for across the 50 holdout sets, and (right) histogram of computed RMSE values across the 50 holdout sets.

Following the assessment of model performance using the holdout validation approach, all available data were used to train a surrogate model used for predictions. To visualize the surrogate model used in this case study, Figure 3-10 shows the surrogate model-predicted partial-variable response functions. That is, the figure shows the predicted surge values when varying one variable (on-diagonal plots) or two variables (off-diagonal plots) while holding other variables at representative values. The representative values are $\Delta p=-55.7, V_{f}=40.1, \theta=-4.1, R_{\max }=68.9, x_{0, \text { lat }}=-71.3$, and $x_{0 . \text { lon }}=40.8$. The surrogate model trained on the full data set was used to make the predictions needed to specify the distribution (and, equivalently, to generate CPTs for node $\hat{\eta}$ in Figure 3-3) by assuming that the model prediction is a deterministic function of the hurricane parameters (see Eq. (3.5)).

The marginal distribution $p\left(\varepsilon_{\eta}\right)$ in Eq. (3.2) (or equivalently, the marginal probability table assigned to node $\left.\varepsilon_{\eta}\right)$ is generated by assuming $\varepsilon_{\eta}$ is equal to the sum of the surrogate model error $\left(\varepsilon_{\eta, S}\right)$ and the error associated with the ADCIRC simulations $\left(\varepsilon_{\eta, A}\right)$ :

$$
\varepsilon_{\eta}=\varepsilon_{\eta, S}+\varepsilon_{\eta, A},
$$

where $\varepsilon_{\eta, S}$ is normally distributed with a mean of zero and standard deviation equal to the mean RMSE from surrogate model testing, and $\varepsilon_{\eta, A}$ is assumed to be normally distributed with parameters as defined in NACCS (Nadal-Caraballo et al. 2015); i.e., mean of zero and standard deviation equal to $0.48 \mathrm{~m}$. 

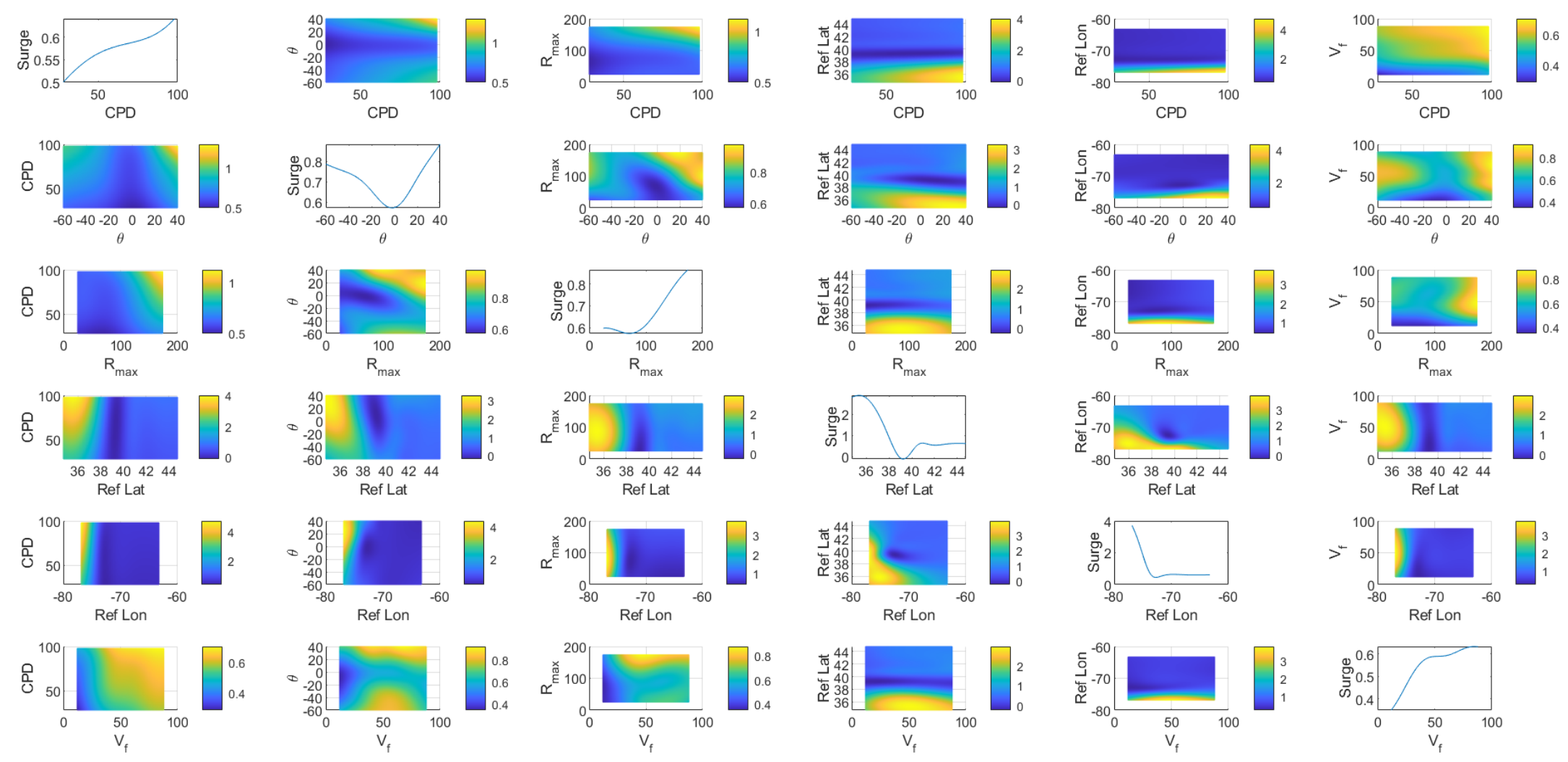

Figure 3-10. Surrogate model-predicted partial-variable response functions. 


\subsubsection{Wind velocity model}

The wind velocity model represents wind velocity $\left(V_{w}\right)$ as a function of a statistical model prediction and a prediction error term $\left(\varepsilon_{V_{f}}\right)$ :

$$
V_{w}=\widehat{V}_{w}+\varepsilon_{V_{w}}
$$

This function is used to define the conditional distribution of $V_{w}$ in Eq. (3.2) (and equivalently, CPT assigned to node $V_{w}$ as a function of $\widehat{V}_{w}$ and $\varepsilon_{V_{w}}$ in Figure 3-3). The conditional distribution for $\widehat{V}_{w}$ is generated using a statistical model that relates wind velocity and central pressure deficit. The statistical equation introduced in NACCS was used to predict maximum wind velocity $(\mathrm{km} / \mathrm{h})$ as a function of central pressure deficit (hPa) (Nadal-Caraballo et al. 2015):

$$
\widehat{V}_{w}=42.4807-0.0084 \Delta p^{2}+2.9752 \Delta p .
$$

Figure 3-11 shows plotted North American Hurricane Database (NOAA 2018) data and the prediction equation developed in NACCS (Nadal-Caraballo et al. 2015) to estimate wind velocity as a function of central pressure deficit.

The marginal distribution $p\left(\varepsilon_{V_{w}}\right)$ in Eq. (3.2) (and equivalently, the marginal probability table for node $\varepsilon_{V_{w}}$ in Figure 3-3) is generated by assuming that $\varepsilon_{V_{w}}$ is normally distributed with zero mean and standard deviation of $18.66 \mathrm{~km} / \mathrm{h}$, consistent with the standard error for the wind velocity prediction equation documented in NACCS (Nadal-Caraballo et al. 2015).

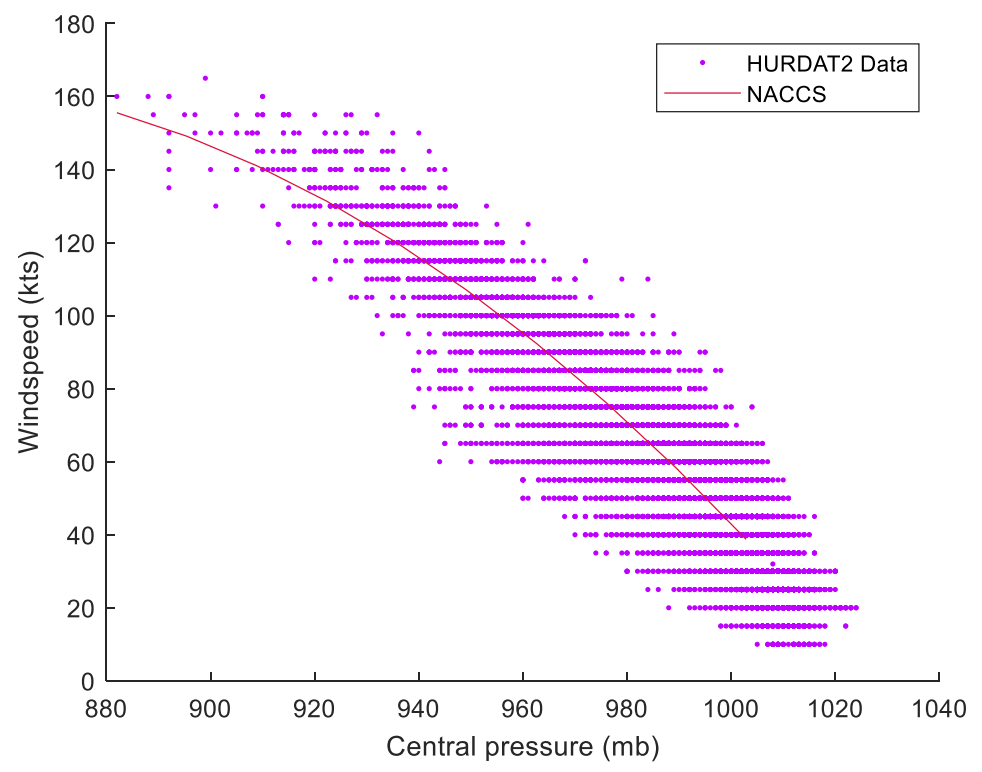

Figure 3-11. Statistical wind velocity prediction model used in this study and plotted North American Hurricane Database (HURDAT2) data.

\subsubsection{Precipitation model}

The precipitation model predicts basin-wide average precipitation $\left(P_{B A}\right)$ as a function of a statistical model prediction and a prediction error term $\left(\varepsilon_{P_{B A}}\right)$ : 


$$
P_{B A}=\hat{P}_{B A}+\varepsilon_{P_{B A}}
$$

This function is used to generate the conditional probability distribution $p\left(P_{B A} \mid \hat{P}_{B A}, \varepsilon_{P_{B A}}\right)$ in Eq. (3.2) (and equivalently, to generate the CPT assigned to node $P_{B A}$ in Figure 3-3). The conditional distribution $p\left(\hat{P}_{B A} \mid V_{w}, \theta, x_{0}, V_{f}\right)$ (or equivalently, the CPT for node $\hat{P}_{B A}$ in Figure 3-3) is generated using a statistical model that relates a regional rain field and hurricane track parameters using a multi-part model.

The Tropical Rainfall Measuring Mission rainfall rates (TRR) model suggested by Tuleya et al. (2007) was leveraged, in which the rainfall rate at a point location is estimated as a function of maximum wind speed of the storm and the distance from the hurricane center. The TRR model represents rainfall rate $(R R$; in./day) as a function of the radial distance from the center of the storm and the maximum wind speed using the expression

$$
R R\left(r, V_{w}\right)=\left\{\begin{array}{c}
T_{0}+\left(T_{m}-T_{0}\right)\left(\frac{r}{r_{m}}\right), r<r_{m} \\
T_{m} \exp \left[-\frac{r-r_{m}}{r_{e}}\right], r \geq r_{m}
\end{array}\right.
$$

where $r$ is the radius from hurricane center $(\mathrm{km})$ to the point of interest (i.e., the point at which the rainfall rate will be predicted). The remaining quantities in Eq. (3.10) are coefficients defined as

$$
\begin{aligned}
& T_{0}=a_{1}+b_{1} U, \\
& T_{m}=a_{2}+b_{2} U, \\
& r_{m}=a_{3}+b_{3} U, \\
& r_{e}=a_{4}+b_{4} U,
\end{aligned}
$$

where $r_{m}$ is representative of the radial extent of the inner core rain rate, and $r_{e}$ is a measure of the radial extent of tropical cyclone rainfall. $T_{0}$ is representative of rain rate at $r=0$, and $T_{m}$ is representative of maximum rain rate at $r=r_{m}$. $U$ is the normalized wind velocity $(\mathrm{kn})$ described by

$$
U=1+\left(V_{w}-35\right) / 33
$$

\begin{tabular}{|c|c|}
\hline$a_{1}=-1.1$ in.day $^{-1}$ & $b_{1}=3.96$ in.day $^{-1}$ \\
\hline$a_{2}=-1.6$ in.day $^{-1}$ & $b_{2}=4.8$ in. day $^{-1}$ \\
\hline$a_{3}=64.5 \mathrm{~km}$ & $b_{3}=-13.0 \mathrm{~km}$ \\
\hline$a_{4}=150 \mathrm{~km}$ & $b_{4}=-16.0 \mathrm{~km}$ \\
\hline
\end{tabular}

where $V_{w}$ is the maximum windspeed (kn). The coefficients $a_{1}$ through $a_{4}$ and $b_{1}$ through $b_{4}$ were obtained by Tuleya et al. (2007) using Tropical Rainfall Measuring Mission rainfall profiles. Table 3-3 shows bias-corrected coefficients using rain gage data (Marks et al. 2002).

Table 3-3. Bias-corrected constants for the TRR model.

Figure 3-12 shows the variation of rainfall rate $(R R)$ with radial distance from the storm center using the TRR model (Tuleya et al. 2007). As shown in Eq. (3.10) and Figure 3-12, when $r$ is smaller than $r_{m}, R R$ increases linearly with radial distance and then decreases exponentially for $r$ values higher than $r_{m}$. In Eqs. (3.11)-(3.14), $U$ is the normalized wind speed, with a maximum value of $115 \mathrm{kn}$. The RMSE of the 
model fitted to Tropical Rainfall Measuring Mission rainfall rates using Eqs. (3.11)-(3.14) was estimated by Tuleya et al. (2007) as 0.28 in.day $^{-1}$.

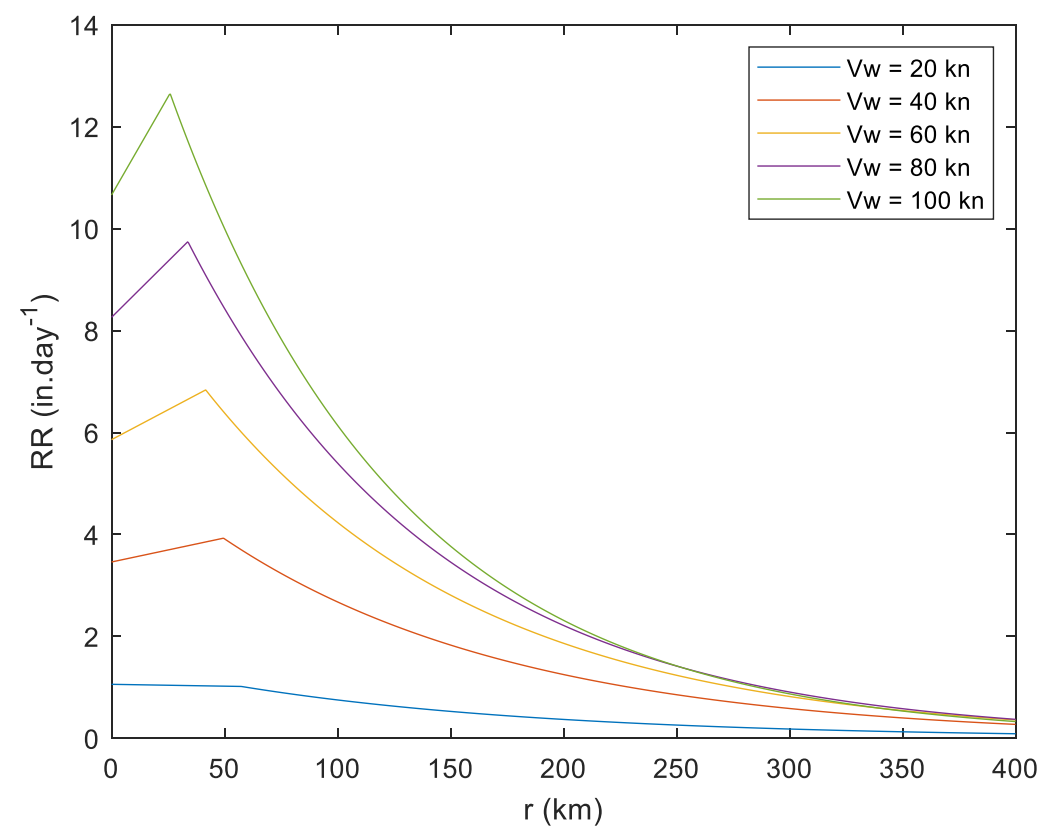

Figure 3-12. Decay of rainfall rate with radial distance using a TRR model (Tuleya et al. 2007).

A TRR model was used to predict rainfall at discrete gridded points across the watershed upstream of the case study location. The grid is shown in Figure 3-13. It consists of 1,454 grid points on a $4 \times 4 \mathrm{~km}$ grid. To estimate storm rainfall, a discretized hurricane track containing information about the location of the hurricane at $1 \mathrm{~h}$ time steps after landfall was created. These spatial and temporal track parameters were calculated as a function of hurricane speed (forward velocity), landfall location, and heading. Then, the radial rain field was computed using the aforementioned TRR model. However, the TRR model provides rainfall rates per day, whereas the basin-wide rain field was estimated at $1 \mathrm{~h}$ time steps. Therefore, daily point rainfall rates produced by the model were scaled (by a factor of 24) to produce rates of inches per hour. 


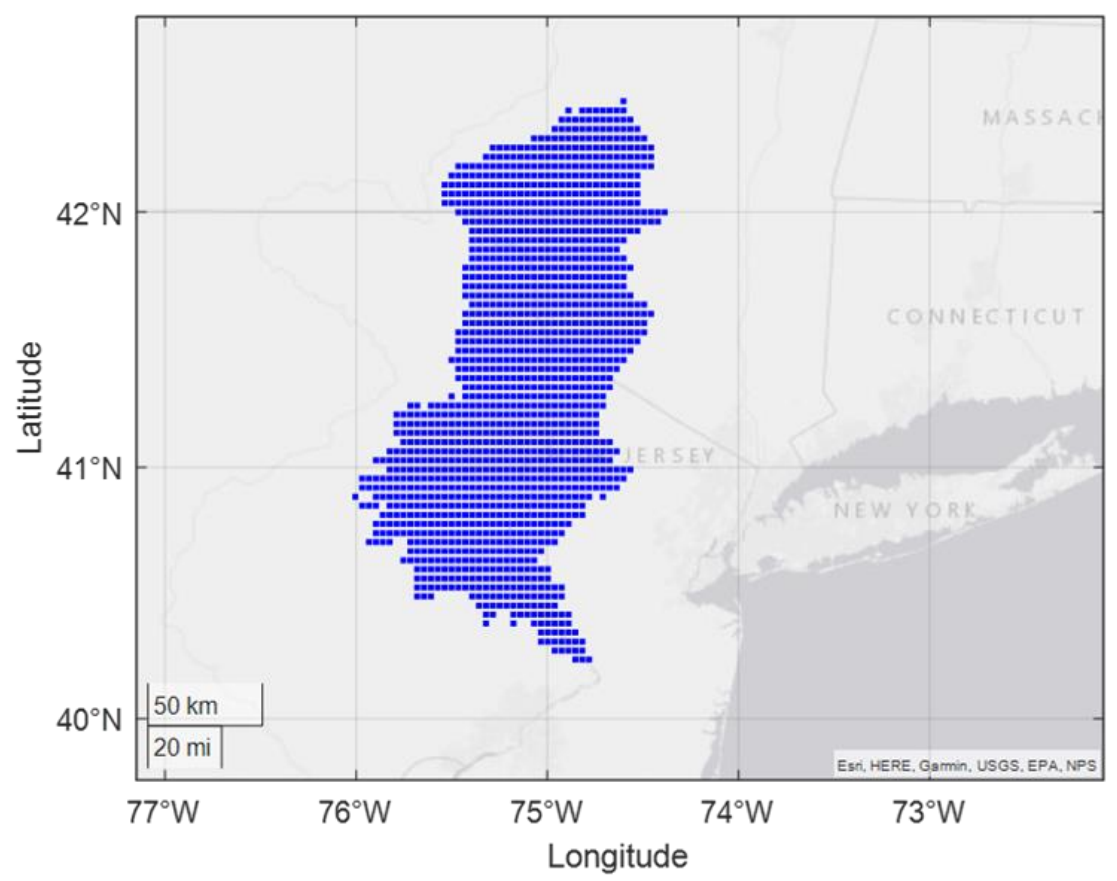

Figure 3-13. Gridded points in the watershed upstream of case study location.

Because maximum wind velocity decays after landfall, and to better capture spatial-temporal changes in precipitation rates, a wind decay model was incorporated into the hurricane-induced precipitation model. The wind decay model used in this study was suggested by Kaplan and DeMaria $(1995 ; 2011)$ and is represented by

$$
V(t)=V_{b}+\left(R V_{0}-V_{b}\right) e^{-\alpha t},
$$

where $V(t)$ is the wind velocity (kn) at time $t$ after landfall, and $V_{b}$ and $\alpha$ are estimated parameters. $R$ is a reduction factor related to increased roughness of the land surface, and $V_{0}$ is the maximum wind speed at landfall. The wind velocity output of Eq. (3.16) was converted from knots to kilometers per hour for subsequent calculations. Table 3-4 shows the RMSE (kn) and coefficients of wind decay model for the area under study suggested by (Kaplan and Demaria 2001). Figure 3-14 presents two examples of hurricane track wind decay using the model in Eq. (3.16), with wind speeds presented in kilometers per hour.

Table 3-4. Wind decay model coefficients for the area under study.

\begin{tabular}{|c|c|c|c|}
\hline$R M S E(\mathrm{kn})$ & $R$ & $\alpha\left(\mathrm{h}^{-1}\right)$ & $V_{b}(\mathrm{kn})$ \\
\hline 11.4 & 0.9 & 0.187 & 29.6 \\
\hline
\end{tabular}




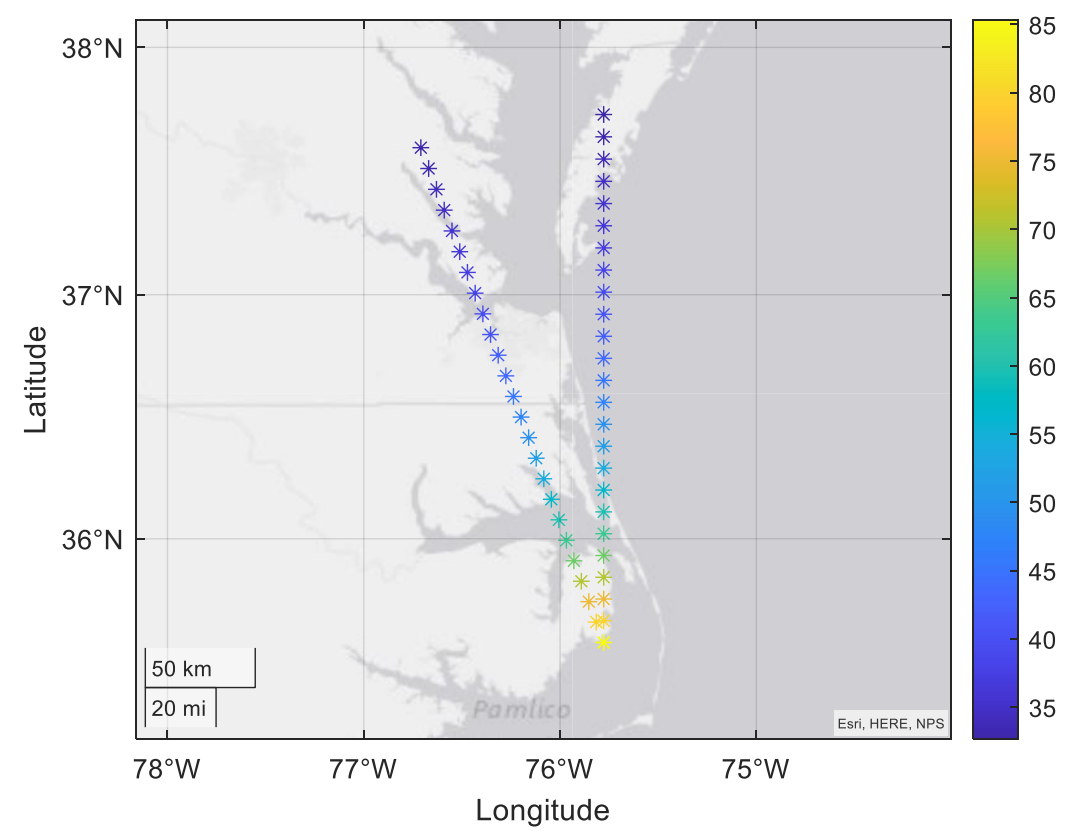

Figure 3-14. Examples of hurricane track wind decay; color key represents wind speeds $(\mathrm{km} / \mathrm{h})$.

To illustrate the implementation of the precipitation prediction model, Figure 3-15 shows a synthetic hurricane track along with the rain field over the watershed at six example discrete time steps. The total rainfall over the watershed during the $24 \mathrm{~h}$ following landfall was computed as the sum of the hourly rainfall quantities at each point. Figure 3-16 shows the total $24 \mathrm{~h}$ precipitation for the track shown in Figure 3-15. Then, the basin average precipitation $\left(\hat{P}_{B A}\right)$ was computed by averaging over all grid points.
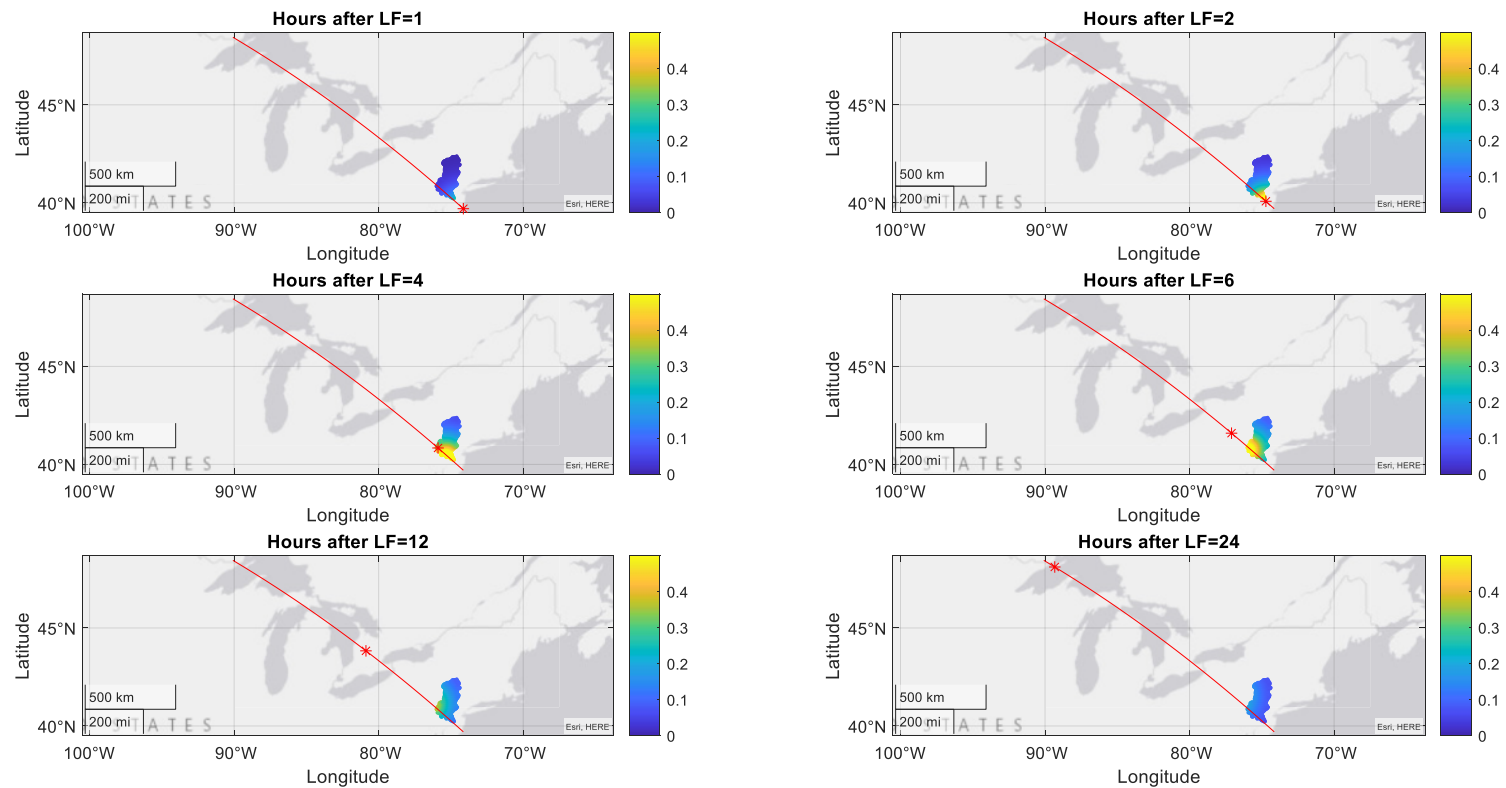

Figure 3-15. Hurricane-induced precipitation in the upstream area for an example storm track (red) at discrete time steps after storm landfall; the red star represents storm center at specific points after landfall; the color key represents rainfall (in./h). 


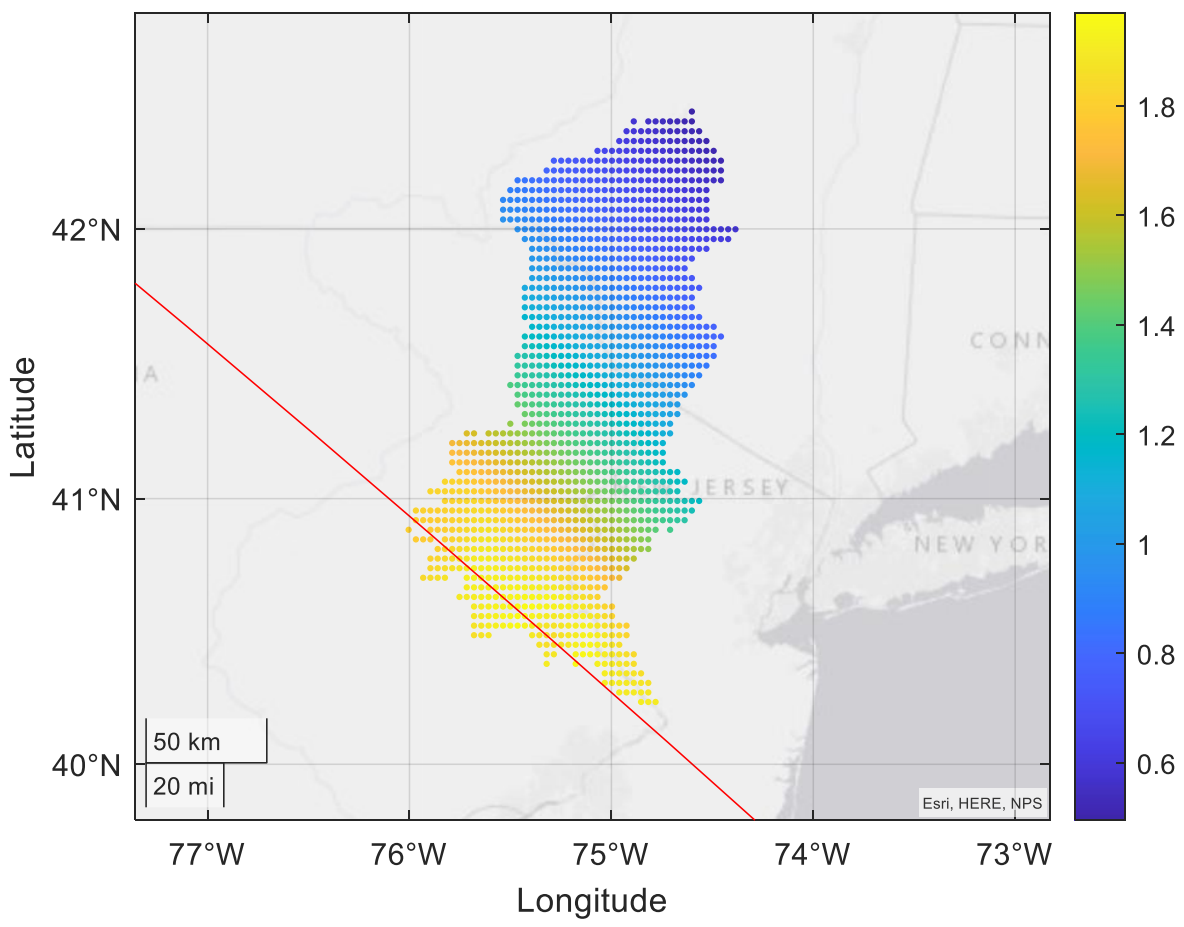

Figure 3-16. Total daily rainfall for the upstream watershed for an example storm track (red); the color key represents rainfall (in./day).

The model error term $\left(\varepsilon_{P_{B A}}\right)$ (see Eq. (3.9)) is assumed to be normally distributed with mean zero and standard deviation equal to the RMSE of the TRR model. Consideration of a more sophisticated approach to defining this error, accounting for error in precipitation and wind decay models, is identified as a potential area for future research.

\subsubsection{Precipitation-induced discharge model}

The precipitation-induced discharge model represents estimates in river discharge caused by upstream hurricane-induced precipitation. It takes the form

$$
Q_{P}=\hat{Q}_{P}+\varepsilon_{Q_{P}},
$$

where $\widehat{Q}_{P}$ is the predicted change in river discharge (cfs) caused by precipitation, and $\varepsilon_{P}$ is a prediction error term. This function is used to generate the distribution $p\left(Q_{P} \mid \widehat{Q}_{P}, \varepsilon_{Q_{P}}\right)$ in Eq. (3.2) (or equivalently, the CPT assigned to node $Q_{P}$ in Figure 3-3). $\hat{Q}_{P}$ is predicted as a function of the upstream basin-wide average precipitation $\left(P_{B A}\right)$ :

$$
\widehat{Q}_{P}=g\left(P_{B A}\right) .
$$

The predictive model in Eq. (3.18) was developed using daily precipitation and runoff data extracted from RAPID (David et al. 2011) and VIC (Oubeidillah et al. 2013) from 1980 to 2015. Figure 3-17 shows the time series of runoff (top panel) and precipitation (bottom panel) for the noted time period, as well as the time series filtered to include only the portions of the time series during hurricane season (orange). This hurricane season-filtered data set was used in the surrogate model development. Figure 3-18 shows the scatterplot of runoff and basin-wide average precipitation for "all data" and hurricane-season data. In 
Figure 3-17 and Figure 3-18, the unit of precipitation is millimeters (consistent with original model results), but to estimate runoff in cubic feet per second in subsequent calculations, precipitation was converted to feet.
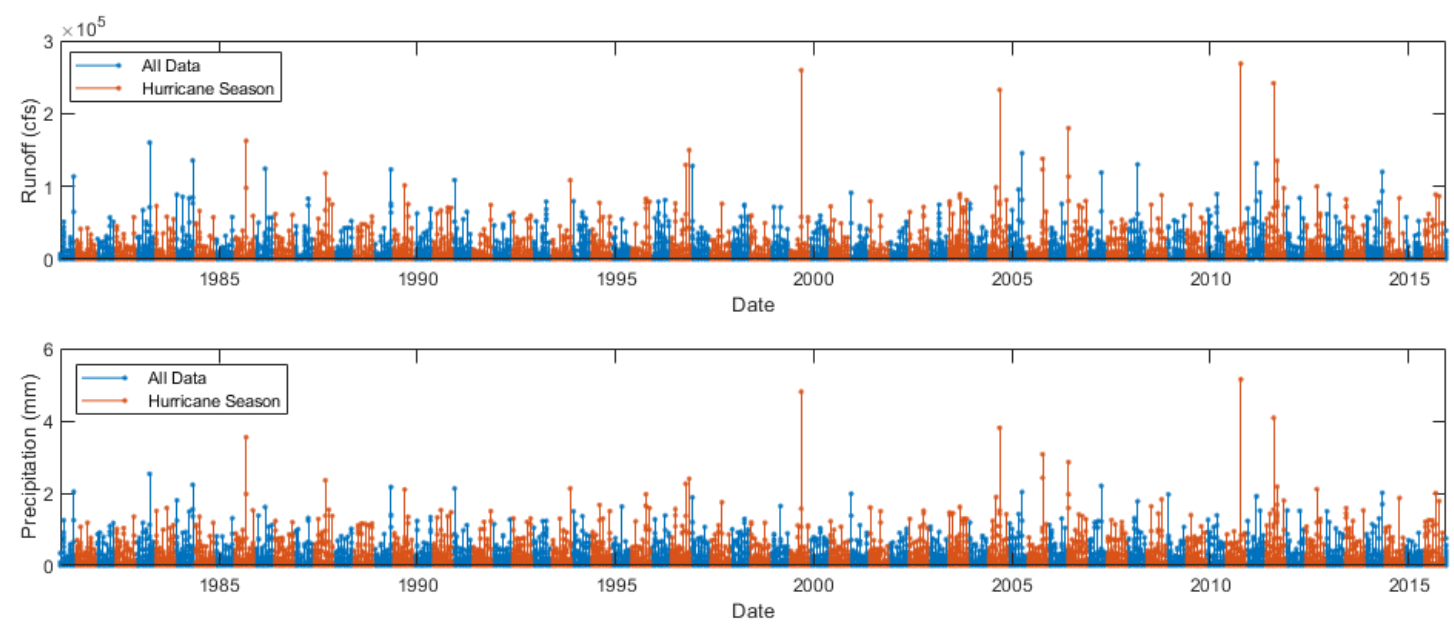

Figure 3-17. Time series of (top) runoff and (bottom) basin-wide average daily precipitation.

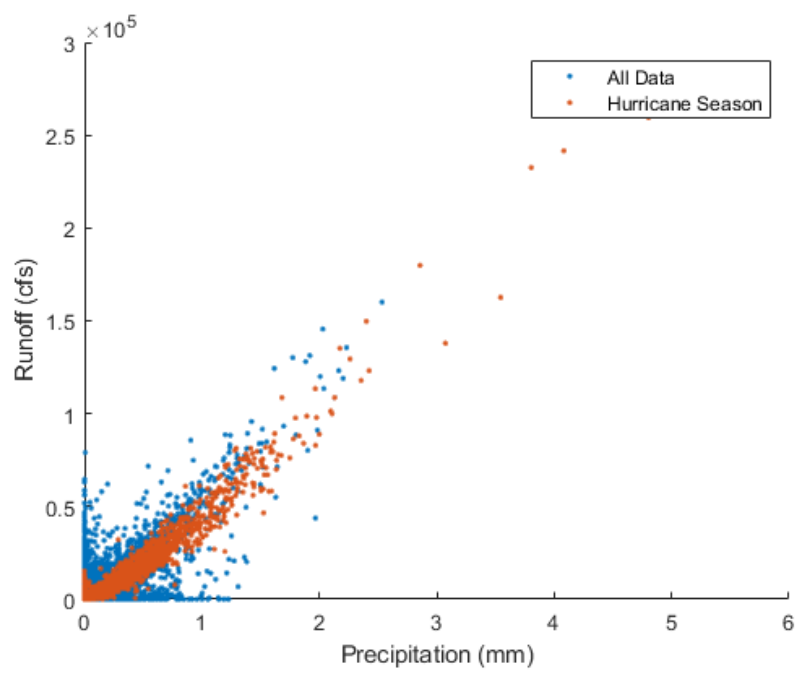

Figure 3-18. Scatterplot of runoff and basin-wide average daily precipitation.

Different predictive models, including generalized linear models and polynomial models, were fitted to data and tested for their performance. The second-degree polynomial model showed the best overall performance. This model takes the form

$$
\hat{Q}_{P}=\alpha_{1}+\alpha_{2} P_{B A}+\alpha_{3} P_{B A}^{2} .
$$

The linear and second-degree polynomial regression lines are plotted along with the input data in Figure $3-19$. The second-order model has a relatively high adjusted $R$-squared value (over 0.95) and an RMSE of $2,970 \mathrm{cfs}$ when computed as a measure of fit. 


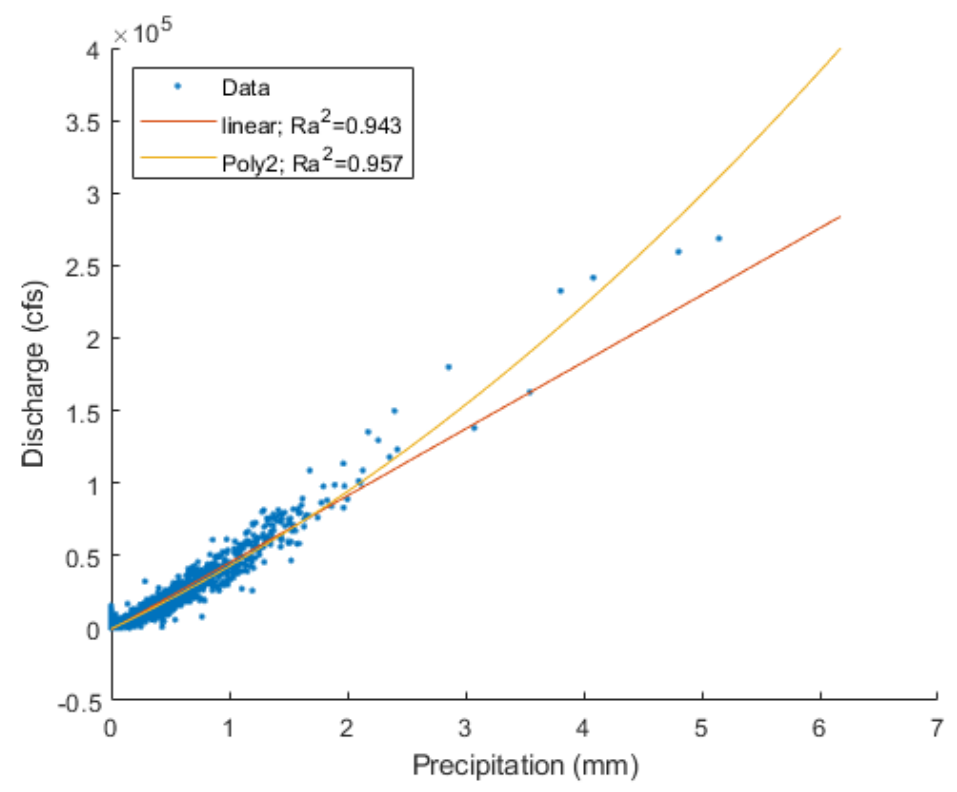

Figure 3-19. Scatterplot of runoff and basin-wide average daily precipitation superimposed with linear and polynomial regression lines.

A holdout validation was performed to assess the variation in RMSE (cfs) and the correlation coefficient $(R)$ between predicted and observed values using 50 holdout folds consisting of a 70/30 split of randomly selected training and testing data. Figure 3-20 (left) shows a scatterplot of surrogate and RAPID-VIC predictions for the out-of-sample predictions across all 50 holdout sets. Figure 3-20 (right) shows a histogram of the computed correlation coefficient across the 50 folds. The correlation coefficients vary from approximately 0.97 to 0.98 .
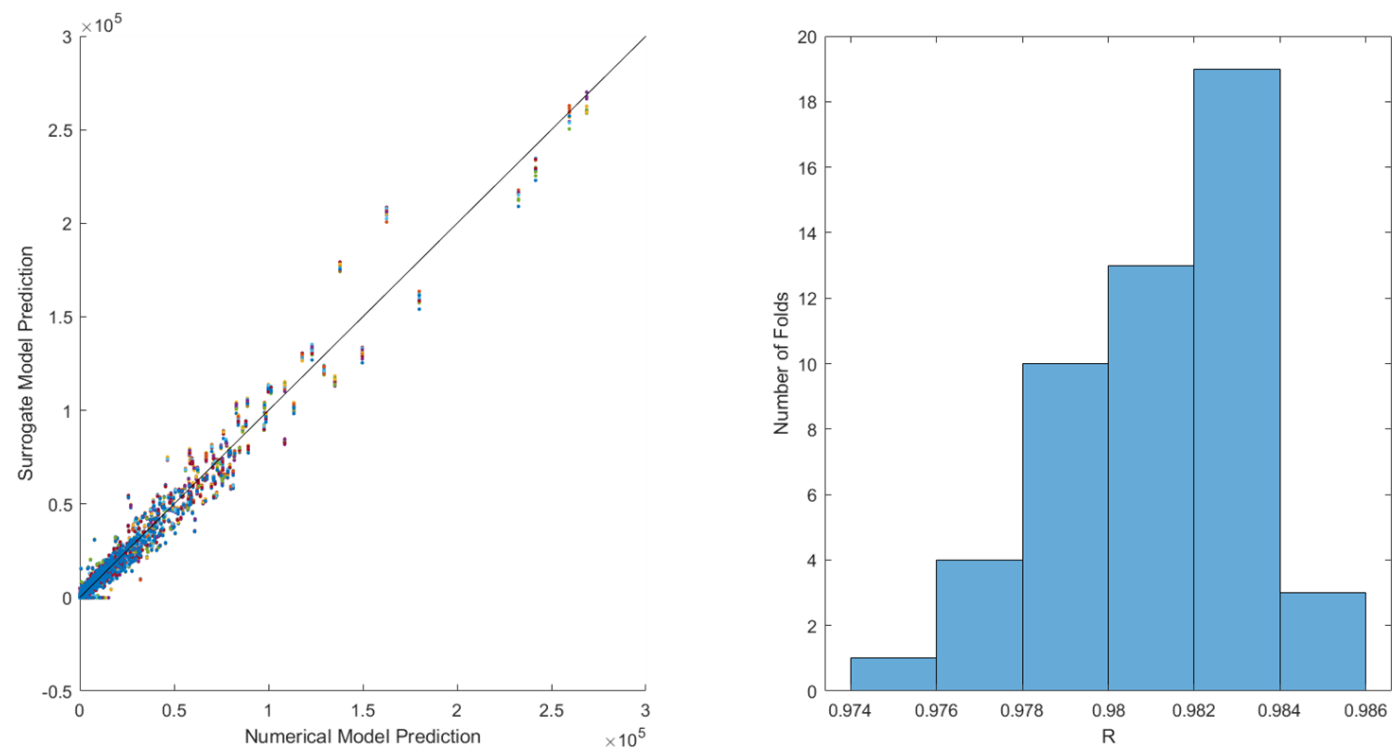

Figure 3-20. (left) Scatterplot of surrogate and numerical (RAPID-VIC) model predictions related to discharge (cfs) for the out-of-sample predictions for the $\mathbf{5 0}$ holdout sets, and (right) histogram of computed correlation coefficients across the 50 holdout sets. 
Figure 3-21 (left) presents a scatterplot of computed out-of-sample prediction errors (RAPID-VIC estimate minus surrogate model prediction), and Figure 3-21 (right) shows the histogram of the calculated RMSEs across the 50 folds. The RMSE varied between approximately 2,300 and 3,100 cfs across the 50 folds, and the mean RMSE was 2,707 cfs. The mean RMSE was used in characterizing the distribution of the model error term used in the analysis. The overall bias (mean error) was relatively small (ranging between approximately -163 and $198 \mathrm{cfs}$ ).
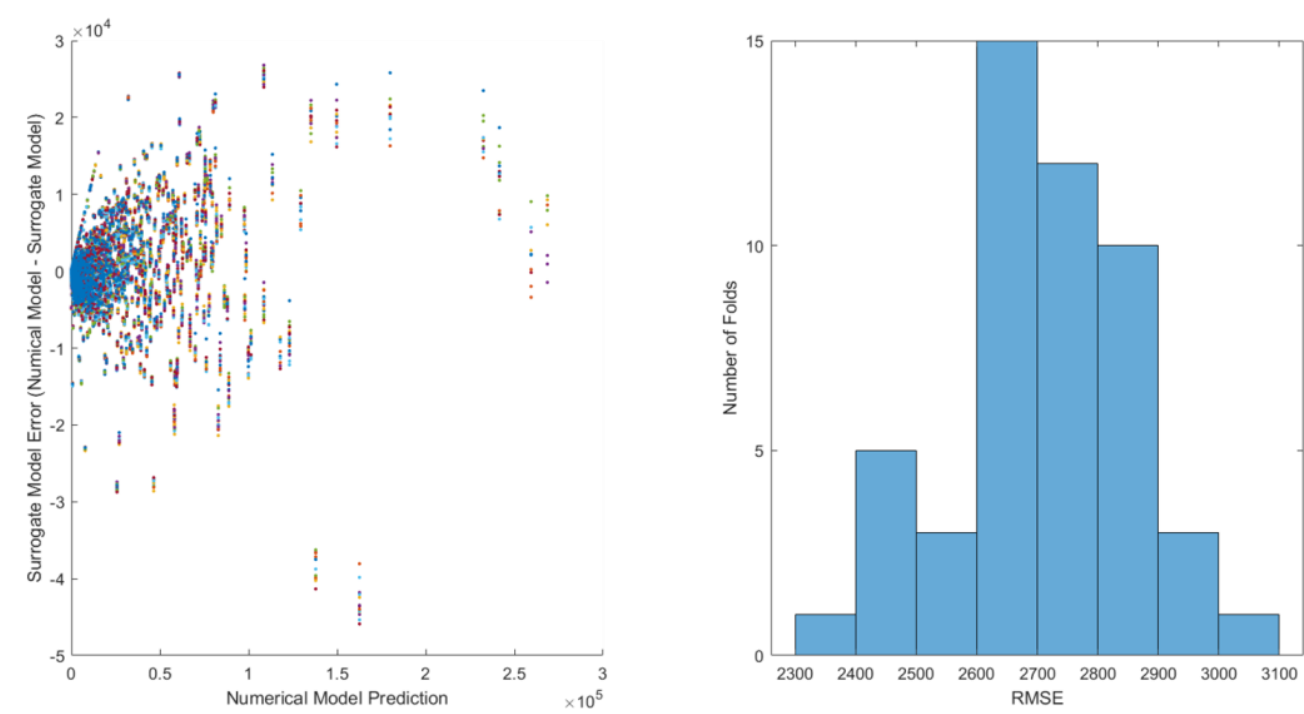

Figure 3-21. (left) Scatterplot of computed out-of-sample prediction error and numerical (RAPID-VIC) model predictions for across the 50 holdout sets, and (right) histogram of computed RMSE values across the 50 holdout sets.

\subsubsection{Surge-, tide-, and river base flow-induced discharge model (combined discharge model)}

The surge-, tide-, and river base flow-induced (combined) discharge model was developed to predict river discharge caused by the simultaneous occurrence of storm surge, tides, and river base flow. It takes the form

$$
Q_{\{\eta, R, T\}}=\widehat{Q}_{\{\eta, R, T\}}+\varepsilon_{Q_{\{\eta, R, T\}}},
$$

where $\hat{Q}_{\{\eta, R, T\}}$ is the (equivalent) total discharge (cfs) caused by surge, river base flow, and tides. $\varepsilon_{Q_{S_{-} R_{-} T}}$ is a prediction error term. To estimate $\hat{Q}_{\{\eta, R, T\}}$, river base flow was converted to equivalent river depth, and surge and tides were added to river base flow-equivalent depth to estimate total water level caused by river surge, tides, and base flow. In the next step, this total water level was converted to discharge using stage-discharge relationship. The predictive model takes the form

$$
\hat{Q}_{\{\eta, R, T\}}=g\left(\eta, T, Q_{R}\right),
$$

where $g\left(\eta, T, Q_{R}\right)$ is a function that "maps" surge, river base flow-equivalent depth, and tide water levels, respectively, to an equivalent discharge. This mapping between water level and an equivalent discharge value was done using a stage-discharge relationship developed for a gage located near the case study location. In the area under study, the error caused by nonlinear interaction between tides and storm surge is negligible (Nadal-Caraballo et al. 2015). Therefore, the simple superposition of tides and surges was judged to generate reasonable results. Equation $\widehat{Q}_{\{\eta, R, T\}}=g\left(\eta, T, Q_{R}\right), \quad$ (3.21) facilitates specification of 
$p\left(\hat{Q}_{\{\eta, R, T\}} \mid \eta, Q_{R}, T\right)$ in Eq. (3.2) (and equivalently, definition of the CPT of node $\hat{Q}_{\{\eta, R, T\}}$ in Figure 3-3). Equation (3.20) also facilitates specification of $p\left(Q_{\{\eta, R, T\}} \mid \hat{Q}_{\{\eta, R, T\}}, \varepsilon_{Q_{\{\eta, R, T\}}}\right)$ in Eq. (3.2) (and equivalently, definition of the CPT of node $Q_{\{\eta, R, T\}}$ in Figure 3-3). Data used in developing the stagedischarge relationship were extracted from USGS 15-min stage-discharge data for the case study location using gage 01463500 located on the Delaware River near Trenton, New Jersey. The gage location is shown in Figure 3-2. Stage-discharge information was available for 14 years, from 2007 to 2020 . The time series of stage and discharge, as well as the scatter plot relating both quantities, are shown in Figure $3-22$.
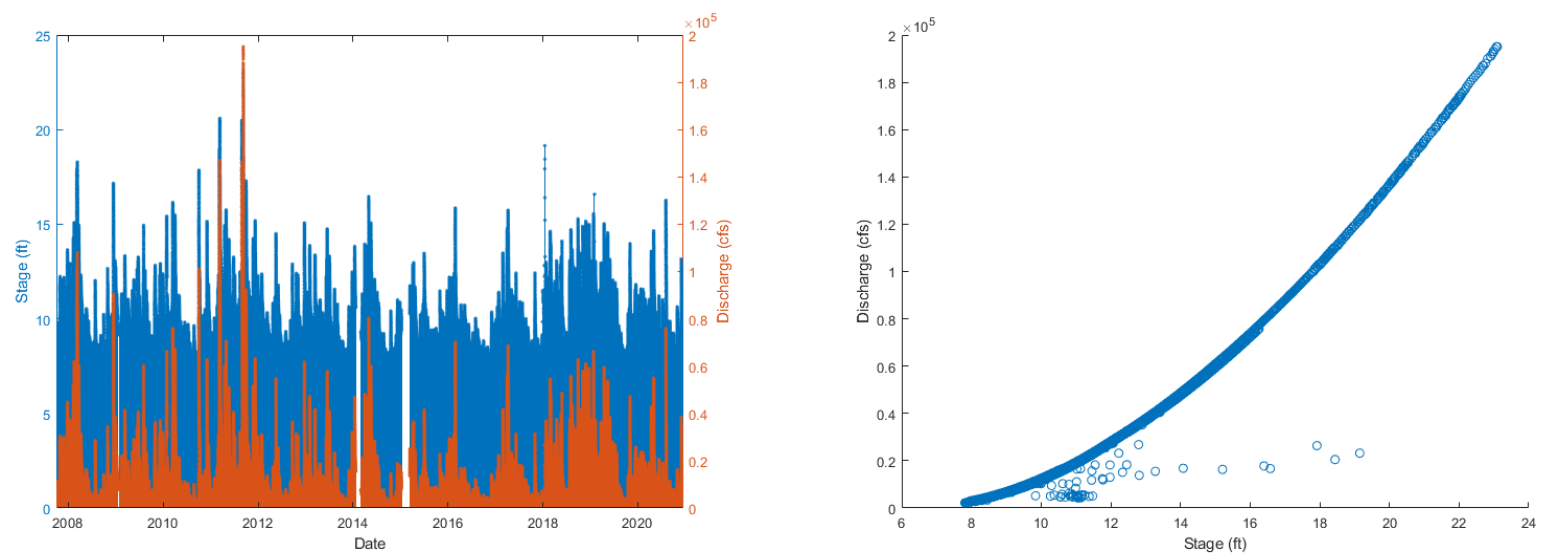

Figure 3-22. (left) Time series of stage and discharge and (right) a scatterplot of surge and discharge.

Given the observed relationship, a simple polynomial model was fitted to the quantities. First- through fourth-order polynomials were fitted to the data, and the resulting functions are shown in Figure 3-23.

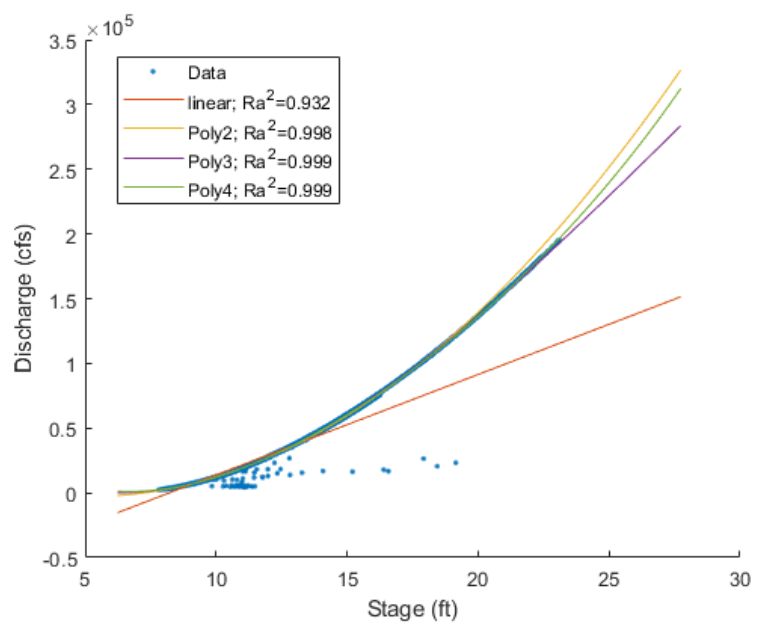

Figure 3-23. Scatterplot of stage and discharge with fitted polynomial models of varying degrees.

The third-order polynomial was selected as the predictive model. This model takes the form

$$
\hat{Q}_{\{\eta, R, T\}}=\alpha_{1}+\alpha_{2} h+\alpha_{3} h^{2}+\alpha_{4} h^{3}
$$


where $h$ is the sum of the river base flow-equivalent stage, tides, and surge. This model has a high adjusted $R$-squared value (nearly 1 ) and an RMSE of $465 \mathrm{cfs}$. However, for several points shown in Figure 3-23, the model did not perform well. These points are highlighted in red in Figure 3-24. As can be seen, a limited number of poor-fit points are related to two distinct segments of the overall time series (Figure 3-24, right).
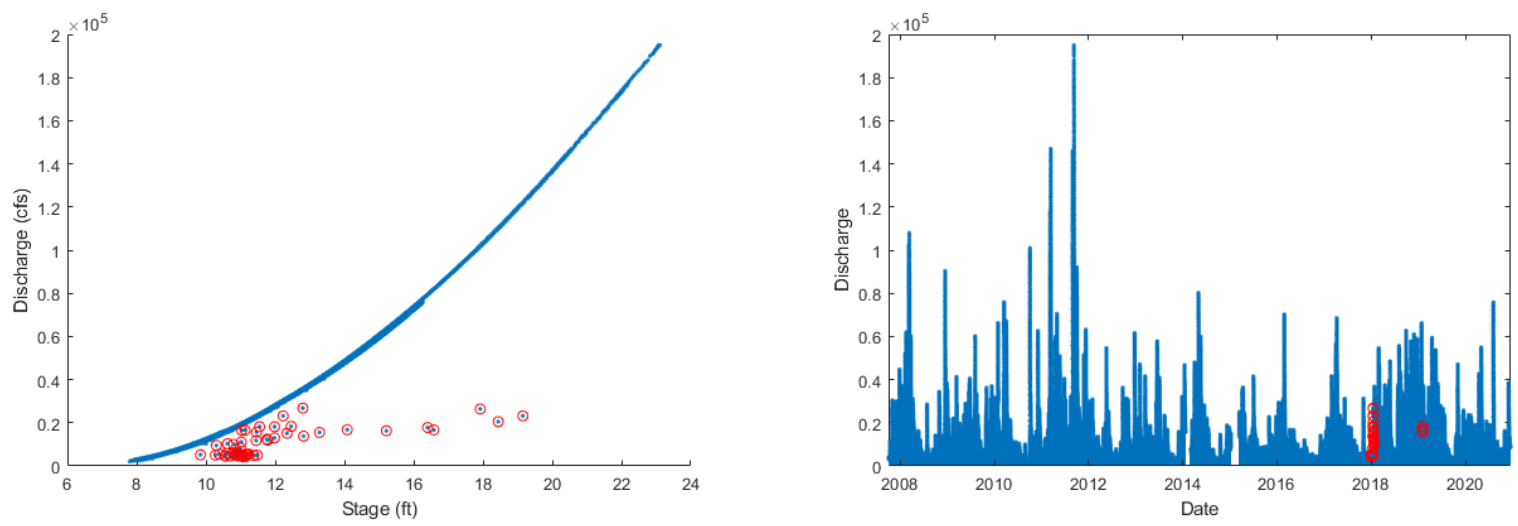

Figure 3-24. (left) Scatterplot of recorded stage and discharge and (right) the recorded time series; poor-fitting points are highlighted in red.

A holdout validation was performed to assess the variation in RMSE (cfs) and the correlation coefficient $(R)$ between predicted and observed values using 50 holdout folds consisting of a 70/30 split of randomly selected training and testing data. The boxplots of the computed RMSE and $R$ values are shown in Figure $3-25$.
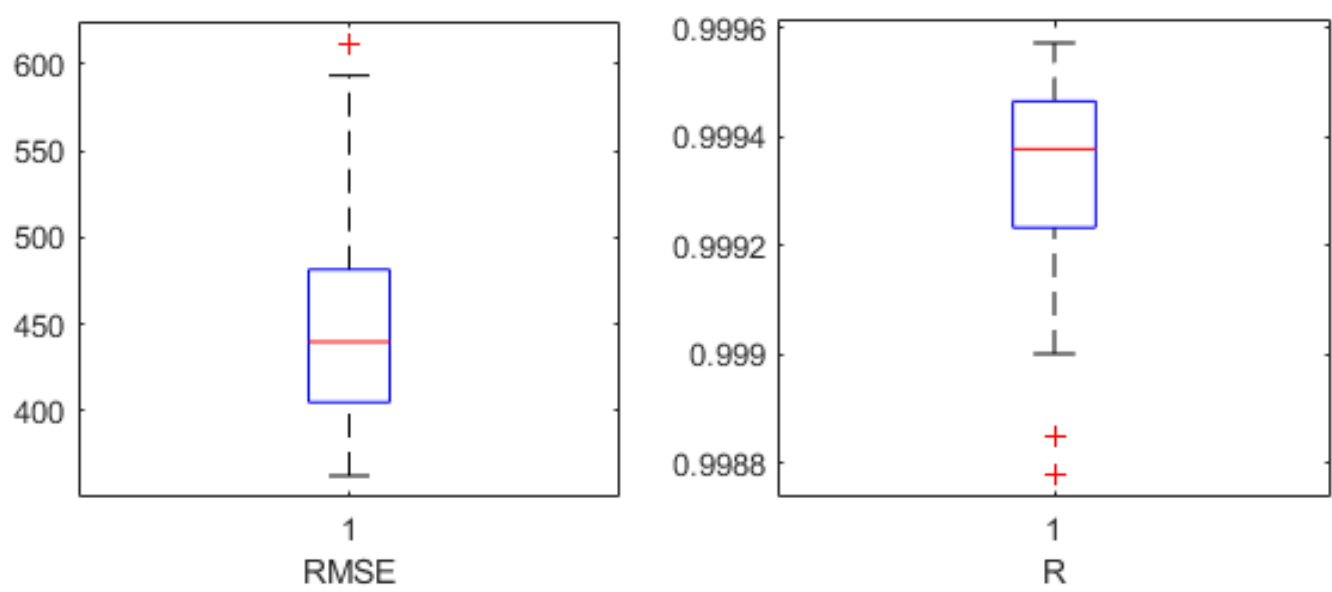

Figure 3-25. Boxplots of (left) RMSE and (right) the correlation coefficient $(R)$ between predicted and observed values for holdout validation.

To develop the marginal distribution for river base flow, $f\left(Q_{R}\right)$ in Eq. (3.2) (or equivalently, the probability table assigned to node $Q_{R}$ in Figure 3-5), a statistical analysis was performed using discharge data available for gage 01463500 located near the case study region (see Figure 3-1). First, the portion of the time series of relevance to the hurricane season was extracted. Then, to capture a "hurricaneindependent" flow, portions of the hurricane record corresponding to dates in which hurricane events were expected to be contributing to river discharge were removed. Then, $5 \%$ of the overall time series was randomly selected to approximate a random sample of discharges; then, a series of candidate 
distributions was fitted to the data set. Figure 3-26 (left) shows the normalized frequency histogram of the selected data set along with PDFs estimated for candidate distributions, and Figure 3-26 (right) shows the empirical CDF for the selected data set along with CDFs estimated for candidate distributions. Table 3-5 shows the estimated AIC and BIC values for the candidate distributions. Ultimately, based on the estimated AIC and BIC and judgment, the LN distribution was selected for modeling the distribution of river base flow (Figure 3-27).
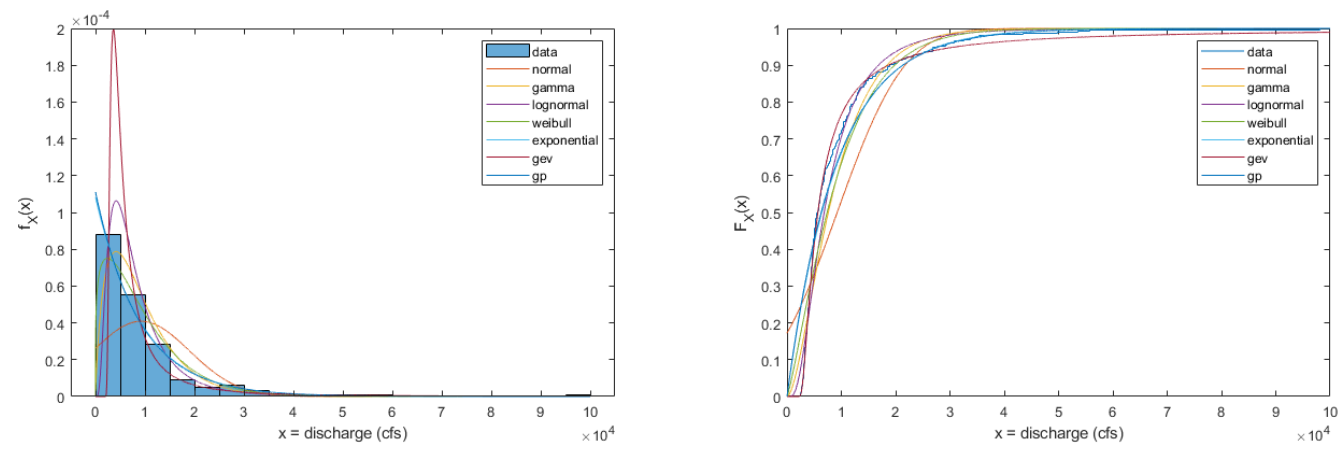

Figure 3-26. (left) Normalized frequency histogram of the selected data set along with PDFs fitted for candidate distributions, and (right) empirical CDF for the selected data set along with CDFs fitted for candidate distributions.
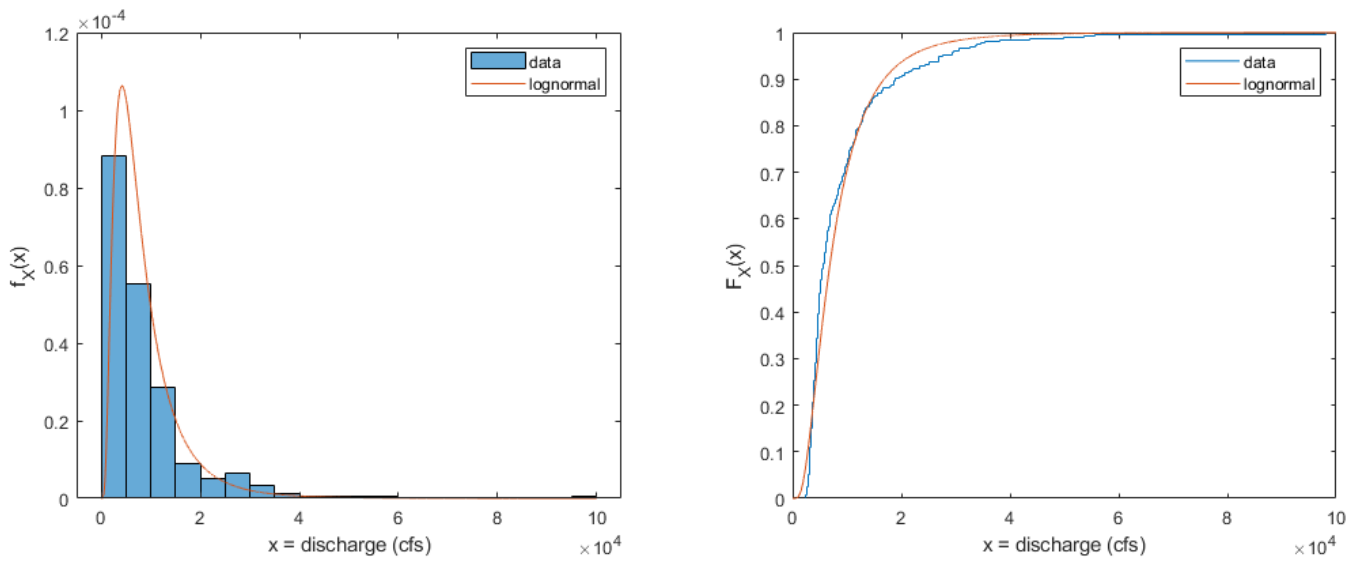

Figure 3-27. (left) Normalized frequency histogram of the selected data set along with LN PDF, and (right) empirical CDF for the selected data set along with LN CDF.

Table 3-5. AIC and BIC values estimated for candidate distributions for base flow.

\begin{tabular}{|c|c|c|}
\hline Distribution name & AIC & BIC \\
\hline GPD & 6194 & 6202 \\
\hline Weibull & 6344 & 6352 \\
\hline NOR & 6663 & 6671 \\
\hline LN & 6219 & 6226 \\
\hline GM & 28108 & 28119 \\
\hline Exponential & 6365 & 6368 \\
\hline
\end{tabular}


To include the effects of tides in the analysis (shown as $T$ in Figure 3-3), predicted tidal elevations from the National Oceanic and Atmospheric Administration (NOAA) water level station located at station 8539993 (Marine Terminal Park, Trenton, New Jersey) were used. Using these data, positive and negative tidal elevations were separated, and empirical CDFs of high (positive) and low (negative) tides were generated. The empirical CDFs were used to generate conditional distributions for water level, given that peak surge occurs during high- and low-tide time periods. A 0.5 probability of peak surge occurring at high tide and at low tide were assumed. That is, $P\left(T=t_{i}\right)=P\left(T=t_{i} \mid\right.$ low $) P($ low $)+$ $P\left(T=t_{i} \mid\right.$ high $) P($ high $)$. Figure 3-28 shows the empirical CDF and PMF for high and low tides. Although the two empirical CDFs were used to generate the conditional PMFs of tide, alternative approaches could be used, including fitting of nonparametric kernel distributions or a multimodal parametric distribution.
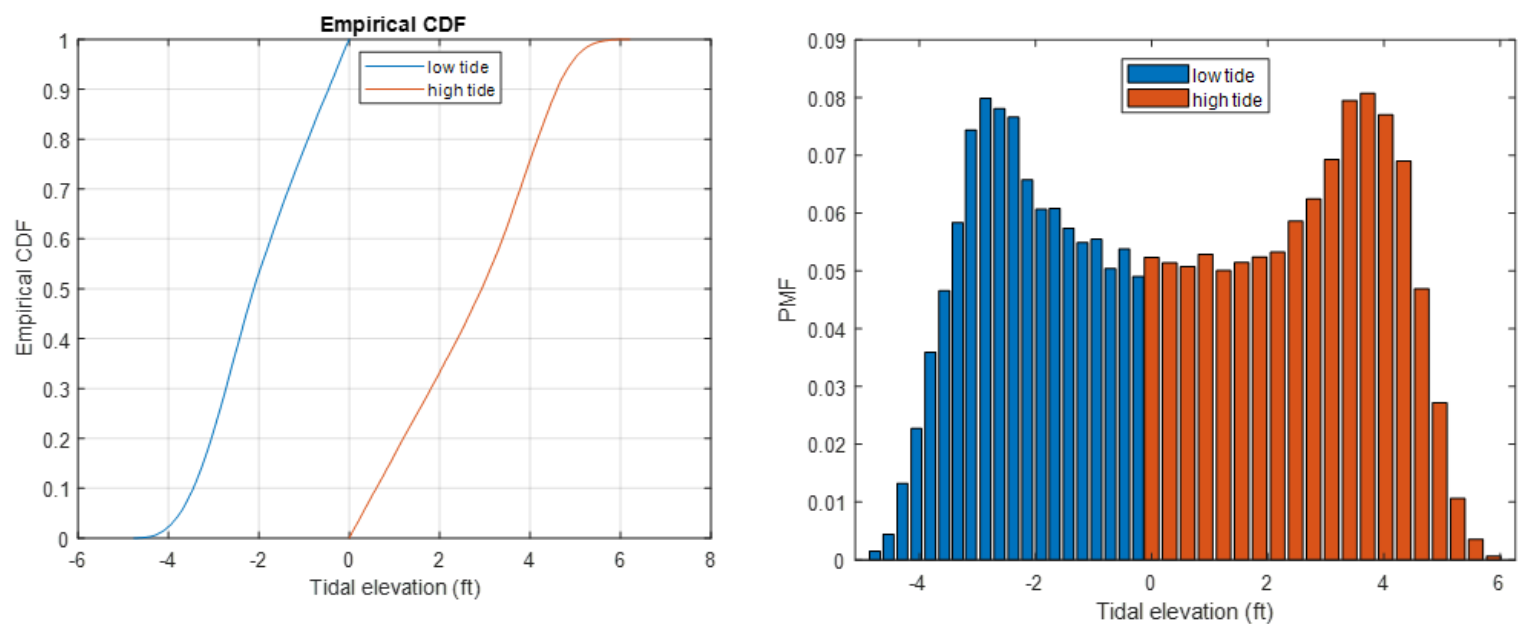

Figure 3-28. Empirical (left) CDF and (right) PMF related to high and low tides.

\subsubsection{Calculation of total discharge}

Total equivalent discharge caused by tides, river base flow, storm surge, and storm-induced precipitation was modeled as the simple superposition of the precipitation-induced discharge and surge-tide-river base flow-equivalent discharge:

$$
Q_{\text {total }}=Q_{\{\eta, R, T\}}+Q_{P}
$$

This simple superposition expression is used to generate the conditional distribution $p\left(Q_{\text {total }} \mid Q_{\{\eta, R, T\}}, Q_{P}\right)$ (and equivalently, conditional probabilities for node $Q_{\text {total }}$ shown in the $\mathrm{BN}$ in Figure 3-3), which facilitates the calculation of $p\left(Q_{\text {total }}>q \mid Q_{\{\eta, R, T\}}, Q_{P}\right)$.

\subsubsection{Model limitations}

This study used a simplified approach that did not account for the complex interactions of physical processes, but which enabled the illustration of the overall Bayesian modeling process. This case study used a strategy in which the sum of river base flow-equivalent stage, tide, and surge were used to generate a water level, and then converted that water level to an equivalent discharge. This discharge was then superimposed with the precipitation-induced discharge to generate a total discharge. In addition to the impacts of "simple superposition," errors were also introduced by the locations of the gages for which data were collected. "Time lags" occurred between the occurrence of peak surge at a location and the 
timing of peak discharge. This study did not account for the differences in timing and instead assumed that the peaks temporally align, which creates a conservative bias in the model.

Superimposing values at the target study location was assumed to be possible. As shown in Figure 3-2, the location of the USGS gage used for developing stage-discharge relationship, the location of save point 5373 used for simulating surge height, and the location of the NOAA tide gage do not coincide. The location of the USGS gage is $1.5 \mathrm{~km}$ upstream of save point 5373. This gage is also located upstream of rock riffles that generally prevent tides from propagating up to the gage station, except in the case of large surges and king tides. Save point 5373 is located downstream of these rock riffles and is affected by tides. Figure 3-29 shows the location of the rock riffles, the USGS gage, and save point 5373. To incorporate the river base flow and for applicability of the stage-discharge relationship developed for the USGS gage location, river characteristics that contribute to the development of the stage-discharge relationship were assumed to be almost unchanged along the $1.5 \mathrm{~km}$ distance between the USGS gage and save point 5373 . Figure 3-29 shows that the river cross-section between the USGS gage and save point 5373 does not change noticeably.

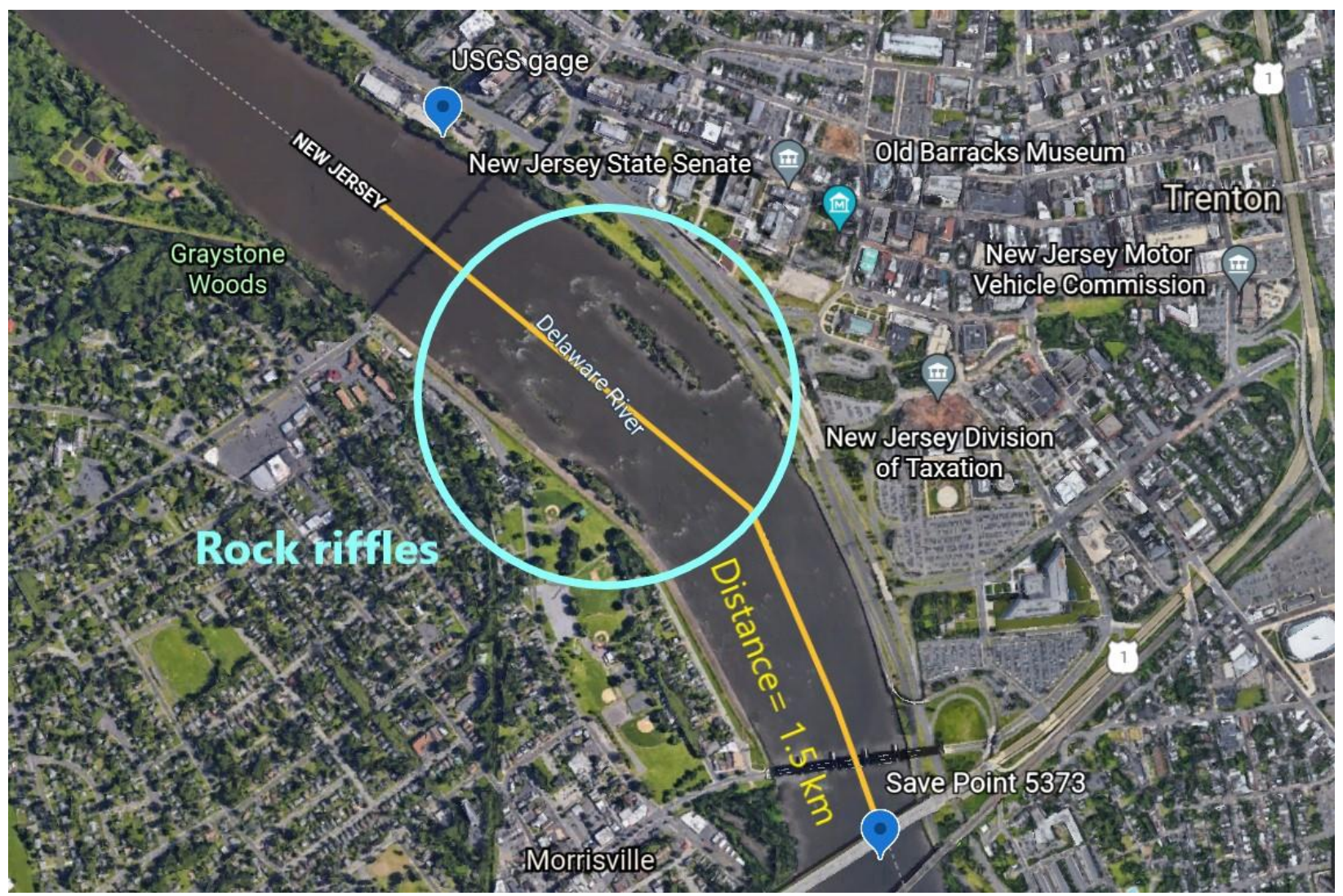

Figure 3-29. The location of USGS gage, rock riffles and save point 5373.

As shown in Figure 3-30, the location of the NOAA tide gage is $2.5 \mathrm{~km}$ downstream of save point 5373 . To analyze how this distance can affect the surge height and propagation of the tides toward the upstream save point 5373, an additional save point 7624 was considered, which is close to tide gage 8539993 . Figure 3-31 shows the ADCIRC-simulated surge height for these two save points across the same storms. The analysis of the surge height at save points 5373 and 7624 showed that similar storms generated similar surge heights at these two points. Considering the similarity between surge heights in these two save points, the tidal levels observed at the location of save point 7624 were added to the surge height simulated at save point 5373 upstream of the tide gage. 


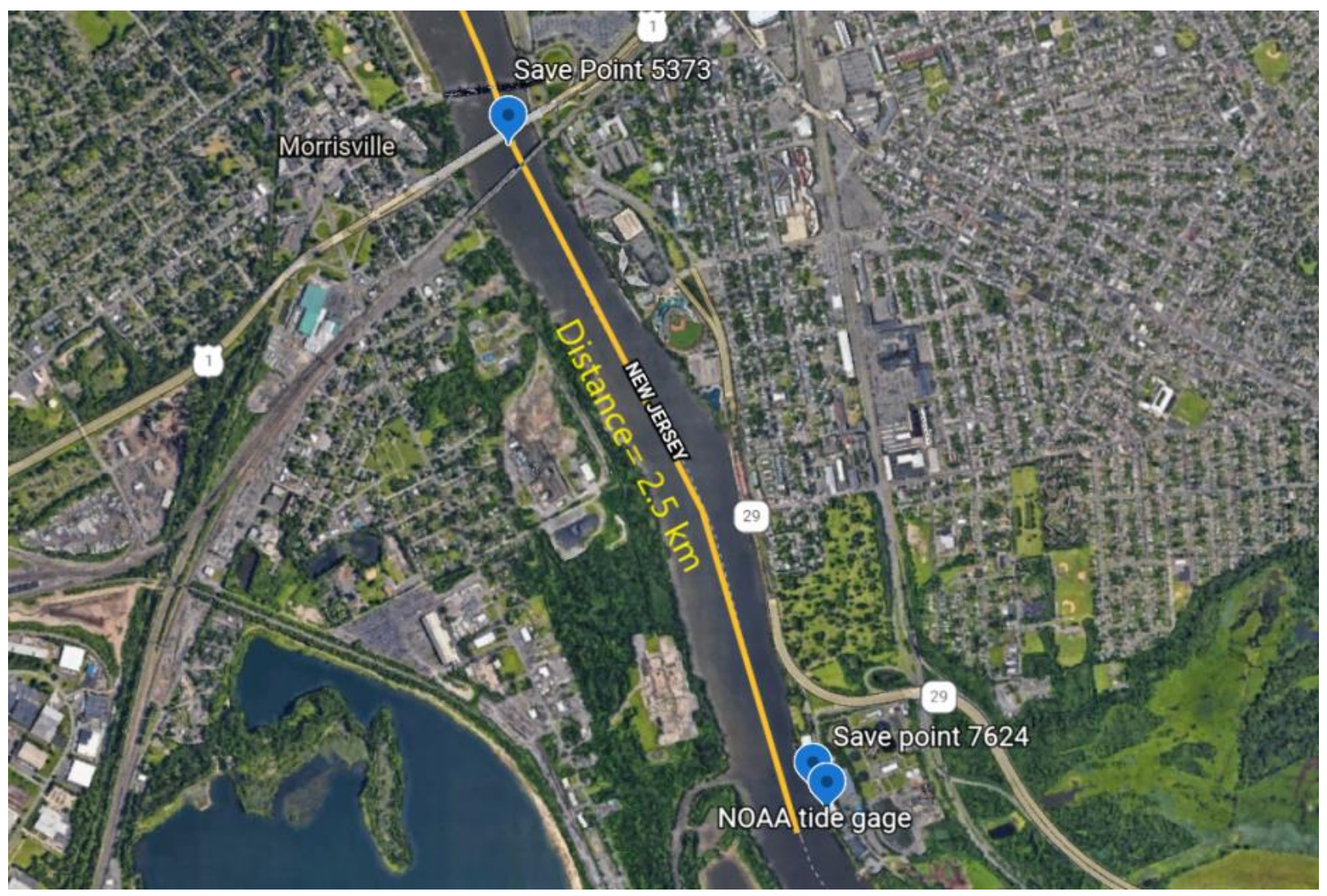

Figure 3-30. Location of save points 5373 and 7624 and the NOAA tide gage.

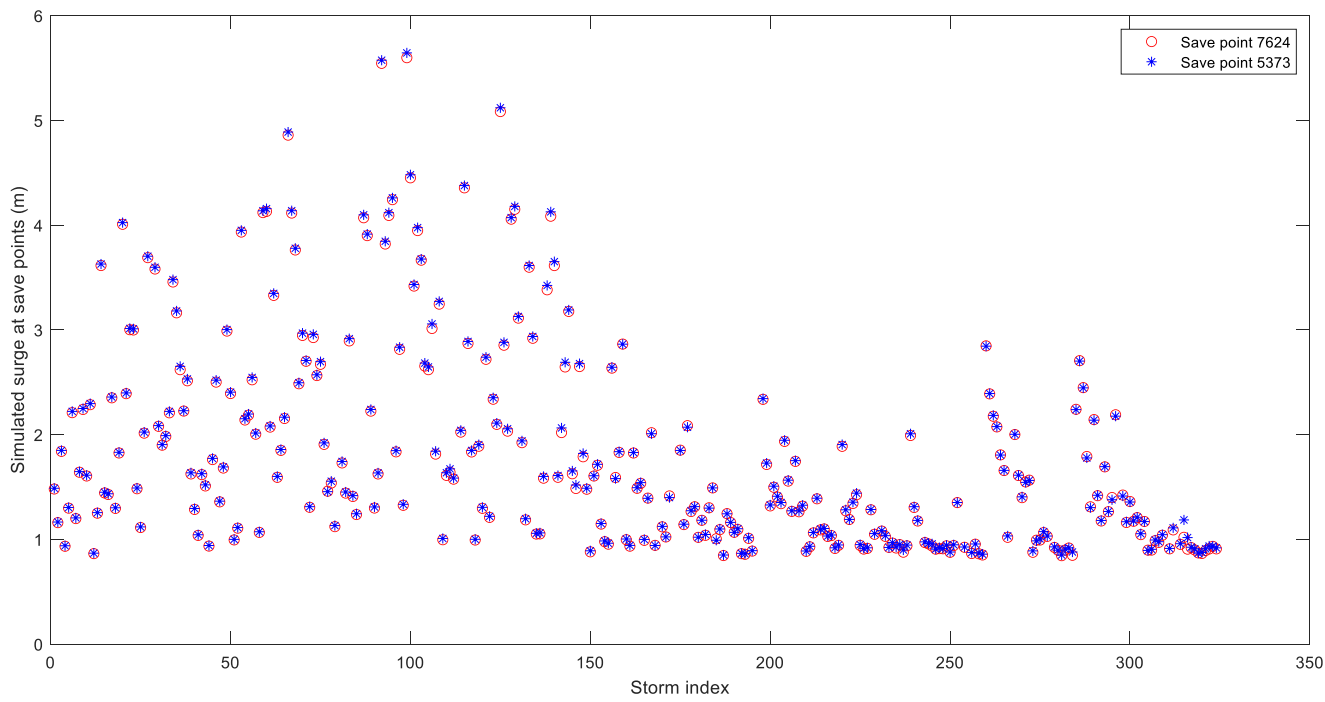

Figure 3-31. ADCIRC-simulated surge height (m) for save points 7624 and 5373. 


\subsubsection{Discretization of distributions}

Consistent with the implementation of the joint probability method in general, Eq. (3.2) (and the BN) requires the discretization of all modeled random variables. Table 3-6 shows discretized values related to constitute random variables.

Table 3-6. Discretized values for the parameters required in the Bayesian formulation.

\begin{tabular}{|c|c|c|}
\hline No. & Variable & Discretized values \\
\hline 1 & $x_{0}$ & $\begin{array}{l}(33.16417,-79.2011),(33.94843,-77.9277),(34.93409,-76.2995),(35.8801,-75.5935), \\
(37.19846,-75.8545),(37.92462,-75.4296),(38.78565,-75.0918),(39.68712,- \\
74.1428),(40.92737,-73.7382),(41.39125,-71.4794),(41.67301,-69.9293), \\
(42.08063,-70.1512),(42.60478,-70.6388),(43.75627,-69.982),(44.47684,-68.1531)\end{array}$ \\
\hline 2 & $\Delta p($ hpa $)$ & $10,23.83,37.66,51.50,65.33,79.16$ \\
\hline 3 & $R_{\max }(\mathrm{km})$ & $0,50,87.50,125,162.50,200$ \\
\hline 4 & $V_{f}\left(\mathrm{~km} \cdot \mathrm{h}^{-1}\right)$ & $0,20,35,50,65,80$ \\
\hline 5 & $\theta\left({ }^{\circ}\right)$ & $-60,-40,-20,0,20$ \\
\hline 6 & $Q_{R}(\mathrm{cfs})$ & 20 values interpolated between 0 and 100,000 at an interval of $5,000 \mathrm{cfs}$ \\
\hline 7 & $T(\mathrm{ft})$ & $\begin{array}{l}-4.763,-4.53,-4.291,-4.05,-3.810,-3.570,-3.340,-3.110,-2.860,-2.620,-2.380, \\
-2.14,-1.90,-1.670,-1.430,-1.190,-0.950,-0.710,-0.470,-0.230,0.310,0.620, \\
0.930,1.240,1.550,1.860,2.170,2.480,2.790,3.10,3.410,3.72,4.030,4.34,4.65,4.96 \text {, } \\
5.27,5.581,5.891\end{array}$ \\
\hline 8 & $\eta(\mathrm{m})$ & 40 values interpolated between 0 and 6 at an interval of $0.15 \mathrm{~m}$ \\
\hline 9 & $P_{B A}\left(\right.$ in.day $\left.^{-1}\right)$ & 15 values interpolated between 0 and 3 at an interval of $0.2 \mathrm{in} . / \mathrm{day}^{-1}$ \\
\hline 10 & $Q_{\{\eta, R, T\}}(\mathrm{cfs})$ & 80 values interpolated between 0 and $950,000 \mathrm{cfs}$ at an interval of $11,875 \mathrm{cfs}$ \\
\hline 11 & $Q_{P}(\mathrm{cfs})$ & 80 values interpolated between 0 and 260,000 at an interval of $3,250 \mathrm{cfs}$ \\
\hline 12 & $Q_{\text {total }}(\mathrm{cfs})$ & 300 values interpolated between 0 and $1,225,500$ at an interval of $4,085 \mathrm{cfs}$ \\
\hline
\end{tabular}

Note: The discretized values presented in row 2 to 12 provide the lower edge (value) of each bin. The values in row 1 provide the landfall location.

To reduce the impact of discretization errors, a Monte Carlo simulation was used in the generation of the CPTs in the BN, which was used to evaluate the expression shown in Eq. (3.2). Although this simulation strategy was used in the context of the BNs, such an approach can be used more generally to reduce discretization errors in the implementation of Bayesian-motivated approaches requiring discretization of the parameter space. Figure 3-32 illustrates the Monte Carlo simulation approach. Under this approach, to generate the CPT for a child node, a Monte Carlo simulation was performed for each combination of the discrete states of its parent nodes, and a conditional distribution was computed and assigned to the child node. For example, in Figure 3-32, a combination of the bins colored in green, purple, orange, and pink represent one combination of the states of the parents to node $\eta$ (excluding landfall location). $N_{\text {sim }}$ simulated values were generated by taking random draws from within the four colored bins, resulting in $N_{\text {sim }}$ combinations of the hurricane parameters. The surge height was estimated for each of these $N_{\text {sim }}$ combinations of hurricane parameters using the surrogate mode. Then, these $N_{\text {sim }}$ surge values were "binned" into the state intervals for node $\eta$, and the result was used to compute the discrete CPT assigned to node $\eta$ for the combination of parent nodes. The process was repeated for all combinations of parent nodes, and the process was executed for all nodes in the BN. 


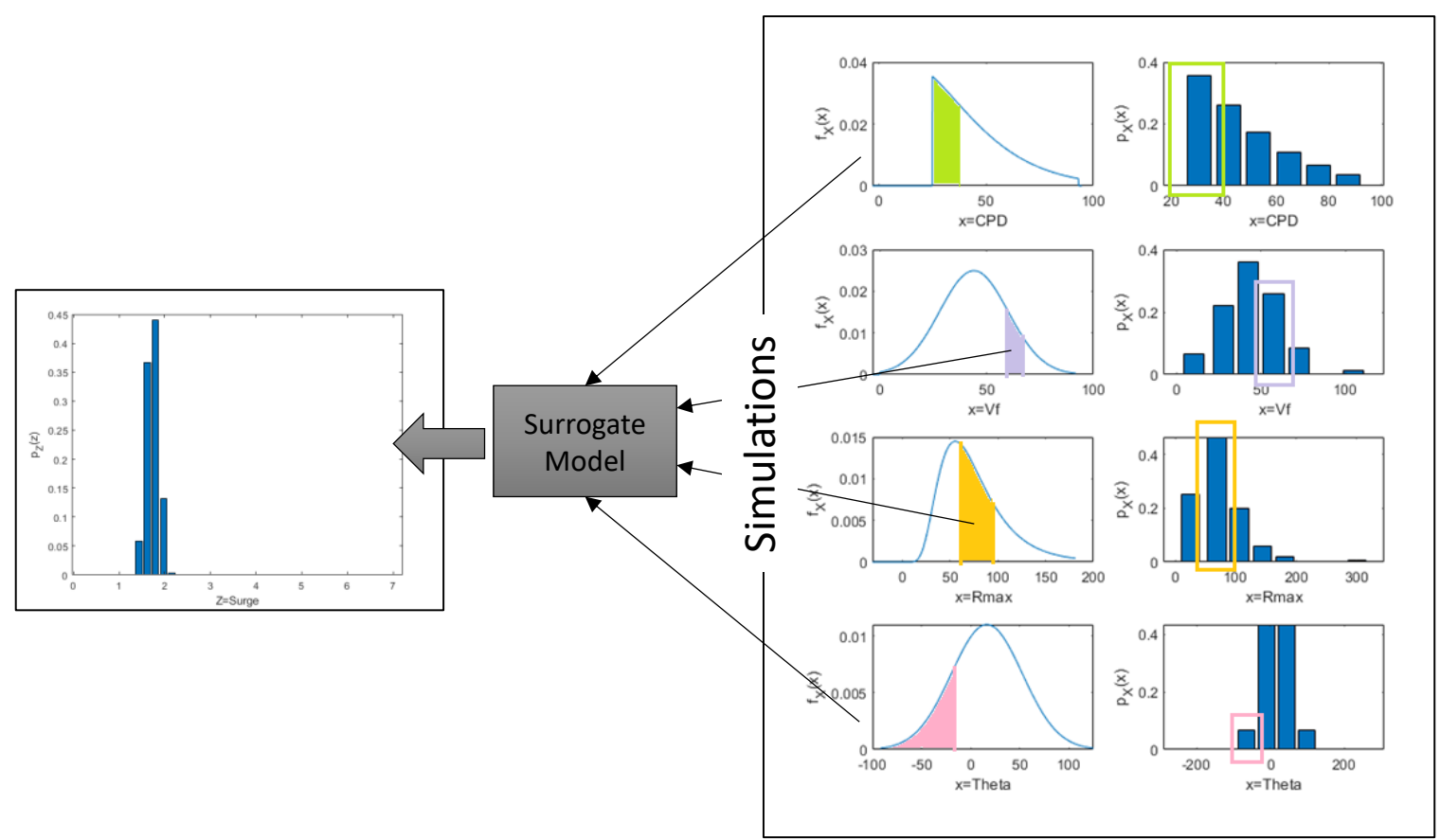

Figure 3-32. Illustration of Monte Carlo simulation approach for generating CPTs.

\subsection{RESULTS}

The capabilities of the BNs were used to "integrate over" (sum over) all nodes shown in the BN in Figure 3-3. The calculation was repeated to solve the discrete form of the integral in Eq. (3.1) for a range of value of $Q_{\text {total }}$. The result was used to generate a hazard curve. For the assumptions described, the resulting illustrative hazard curve is shown in Figure 3-33. To generate this curve, the annual hurricane occurrence rate was assumed to be 0.18 storms per year for the area under study (Nadal-Caraballo et al. 2015). 


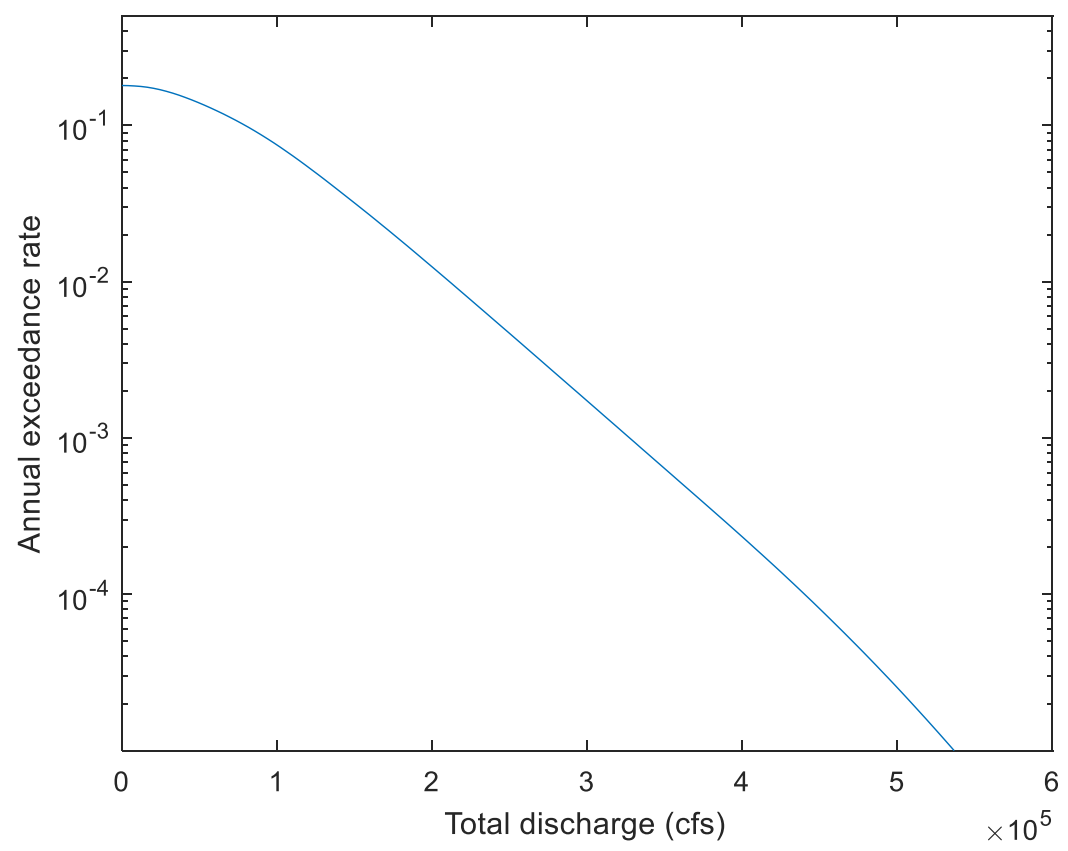

Figure 3-33. Total discharge hazard curve (presented for illustrative purposes).

\subsection{MODEL PERFORMANCE ASSESSMENT}

The last step in this study included a semi-qualitative evaluation to assess the performance of the Bayesian-motivated model against historical events to ensure that the results were reasonable. As noted previously, the goal of this study was to demonstrate the process of using a Bayesian-motivated approach while using a series of simplified empirical, statistical, and surrogate process models. An actual application of the Bayesian-motivated approach would likely replace these simplified models with more robust numerical or analytical models. Nevertheless, an initial performance assessment of the compilation of models used in the case study was performed. The Bayesian-motivated model is intended for probabilistic hazard assessment rather than as a predictive model. Therefore, this performance assessment should not be interpreted as a formal model validation study.

The model was assessed by leveraging the capabilities of the $\mathrm{BN}$ to facilitate information updating. "Evidence" was entered into nodes within the BN (i.e., the states of certain nodes were set as known), and the posterior distributions were extracted and used in the performance assessment. Specifically, by specifying storm parameters, river base flow, and tides associated with observed events, evidence was introduced. Then, the resulting posterior distribution of total discharge was compared with the peak discharge levels observed at the gage 01463500 location on the Delaware River near Trenton, New Jersey. As noted previously, the USGS gage is located $1.5 \mathrm{~km}$ upstream of the target location with intervening rock riffles that limit (but do not prevent) surges and tides from propagating from the study location (save point 5373) to the location of the river gage. Furthermore, the assumption was made that peak surge and discharge temporally coincide. Therefore, the BN model was expected to produce consistently higher discharge values than observed at the river gage for historical storms.

Considering the availability of storm data (especially $R_{\max }$ data, which were only available after 2005 at the time this case study was developed), a limited number of the historical storms occurring in the case study area was available for the performance assessment. Figure 3-34 through Figure 3-36 show the observed storm tracks of three storms affecting the case study region. These figures also include the 
synthetic storm tracked modeled in this study. Table 3-7 shows the corresponding representative storm parameters as well as measured discharge. The representative values are the values of the storm parameters specified in NOAA IBTrACS (International Best Track Archive for Climate Stewardship) database (https://www.ncdc.noaa.gov/ibtracs/) taken at the point before landfall. Figure 3-37 through Figure 3-39 also show the locations at which the representative values were taken. For each storm, these storm parameters were entered as evidence for the corresponding nodes in the $\mathrm{BN}$.

Assumed river base flow values are shown in Table 3-7 (column labeled " $Q_{R}$ "). These values were extracted from the gage record for gage 01463500 by investigating 15 -min stage values and selecting a base flow value prior to the storm affecting the area (e.g., one day prior to storm landfall).

Tidal values were extracted from the record for NOAA tide station 8539993 (Marine Terminal Park, Trenton, New Jersey) by finding the tide level corresponding to the time of the observed peak water level at gage 01463500. Tide values at the time of peak surge are provided in the column labeled "Tide at peak WL." When the time of peak water level occurred for an extend period of time (e.g., $1 \mathrm{~h}$ or more), a representative "midpoint" tide value was selected from the time period. For each storm, the river base flow and tide values were entered in the corresponding nodes in the BN. Model output was highly sensitive to the assumed tide values, and minor changes substantially changed the computed total discharge results.

For each storm, the poster distributions of total discharge were computed using the BN. In Figure 3-41 and Figure 3-42, blue bar plots represent the posterior distribution of modeled discharge values for each storm scenario. In addition to providing the PMF of combined discharge for all three storms, Table 3-7 also provides the "modal bin" of the distribution (i.e., the bin with the highest probability mass in the PMF). As can be seen, the bins associated with the highest probability for all three storms differed from the USGS data. This is partially because of simplifications in modeling that will tend to bias model results higher (e.g., the assumption of temporal alignment of peaks), as well as physical considerations (e.g., the observed values at the USGS gage will not fully account for the contribution of surge because of the presence of the rock riffles).

In general, the model results appear to be reasonable considering the high level of uncertainty involved in the prediction of river discharge using storm parameters, particularly given the limited fidelity of models and assumptions used in this case study. Limitations associated with physical modeling were discussed. Furthermore, an additional source of uncertainty arose from the limited number of landfall locations modeled for the case study region, which is consistent with other probabilistic studies. An approximation in landfall location caused a noticeable increase or decrease in estimated surge height (and thus the equivalent river discharge), which is presented in the partial-variable response functions in Figure 3-10. Furthermore, landfall locations notably affected the track path of the storm and thus the spatial distribution of the rain field leading to runoff. Storms can make landfall at any point along the coast. However, modeling a high number of the points along the coast is computationally expensive for the synthetic tracks used in the probabilistic assessment.

The change in storm characteristic after landfall is another factor contributing to the aggregation of uncertainty in the model. Synthetic tracks modeled in this study did not account for the factors that affect the storm path on land. In the case study, synthetic tracks were generated using an idealized track path (a straight line) defined by a landfall location and heading direction. A different, more realistic, storm path can contribute to more decay of storm intensity and a change in the amount of precipitation affecting the area.

In this study, hourly precipitation values were estimated using the TRR model augmented with a model to decay wind speeds after landfall. However, the TRR model provides the daily precipitation rate and, to 
convert the daily precipitation rate into an hourly rate, the assumption of the uniform hourly distribution of the precipitation was made in this study. Furthermore, there are no well-developed (statistical/analytical) models for the decay of storm speed on the land, which affects the location of the storm at each time and the precipitation received at each point located at the upstream watershed. This study assumed constant forward velocity for the storm on the land, which can underestimate the estimated precipitation by considering the fast movement of the storm while the wind is decaying over time.

Table 3-7. Storm parameters and surge and discharge values related to USGS gage and modeling results.

\begin{tabular}{|c|c|c|c|c|c|c|c|c|c|c|c|c|}
\hline $\begin{array}{l}\text { Name } \\
\text { of } \\
\text { storm }\end{array}$ & $\begin{array}{l}\text { Time of } \\
\text { landfall }\end{array}$ & LAT & LON & $\begin{array}{c}\Delta P \\
(\mathbf{h P a})\end{array}$ & $\begin{array}{c}V_{w} \\
(\mathbf{k m} / \mathbf{h})\end{array}$ & $\begin{array}{c}\theta \\
\text { (degree) }\end{array}$ & $\begin{array}{c}V_{f} \\
(\mathbf{k m} / \mathbf{h})\end{array}$ & $\begin{array}{r}R_{\max } \\
(\mathbf{k m})\end{array}$ & $\begin{array}{c}\text { Tide } \\
\text { at } \\
\text { peak } \\
\text { WL } \\
\text { (ft) }\end{array}$ & $\underset{(\mathbf{c f s})}{Q_{R}}$ & $\begin{array}{l}\text { USGS } \\
Q(\mathbf{c f s})\end{array}$ & $\begin{array}{c}\text { Modal bin } \\
\text { of PMF }{ }^{1} \\
\text { for } \\
\text { discharge }\end{array}$ \\
\hline Isaias & $\begin{array}{c}8 / 4 / 2020 \\
00: 00\end{array}$ & 33.7244 & -78.5834 & 25.25 & 138 & 19 & 37 & 37 & 4.56 & 4,000 & 75,800 & $\begin{array}{c}118465- \\
122550\end{array}$ \\
\hline Fay & $\begin{array}{c}7 / 10 / 2020 \\
18: 00\end{array}$ & 39.5473 & -74.3161 & 15.25 & 92 & 7 & 25 & 166 & -1.86 & 4,500 & 19,000 & $\begin{array}{c}44935- \\
49020\end{array}$ \\
\hline Irene & $\begin{array}{c}\text { 8/28/2011 } \\
09: 00\end{array}$ & 39.1783 & -74.49 & 55.25 & 111 & 20 & 42 & 185 & 5.25 & 15,000 & 146,000 & $\begin{array}{l}236930- \\
241015\end{array}$ \\
\hline
\end{tabular}

${ }^{1} \mathrm{Bin}$ associated with the highest probability mass $\mathrm{WL}=$ water level

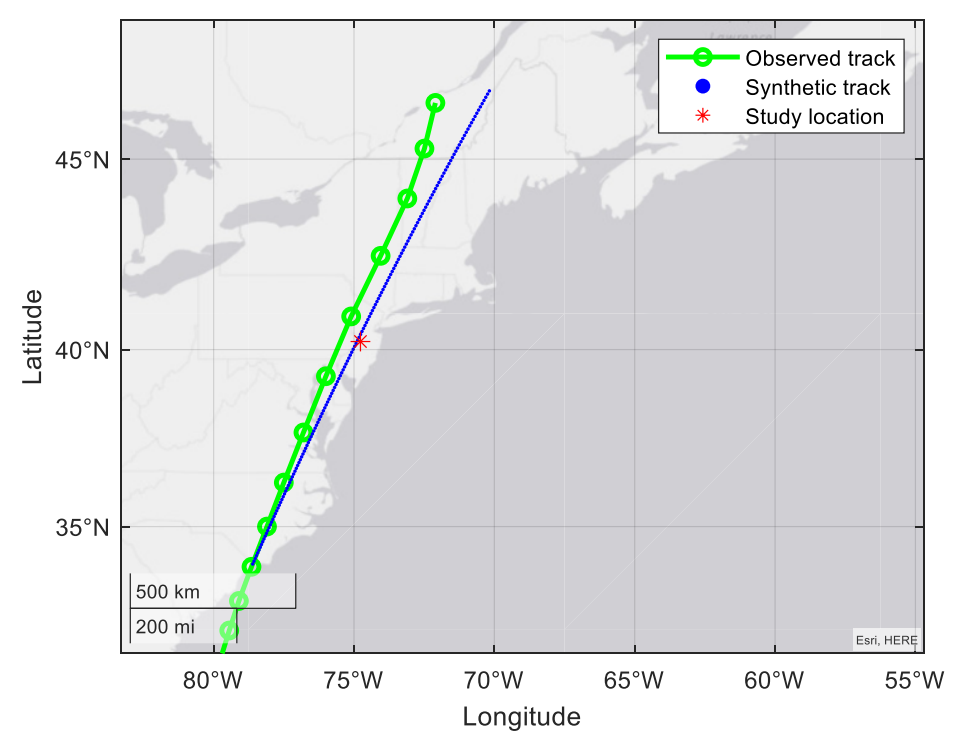

Figure 3-34. Synthetic and observed storm track related to Hurricane Isaias. 


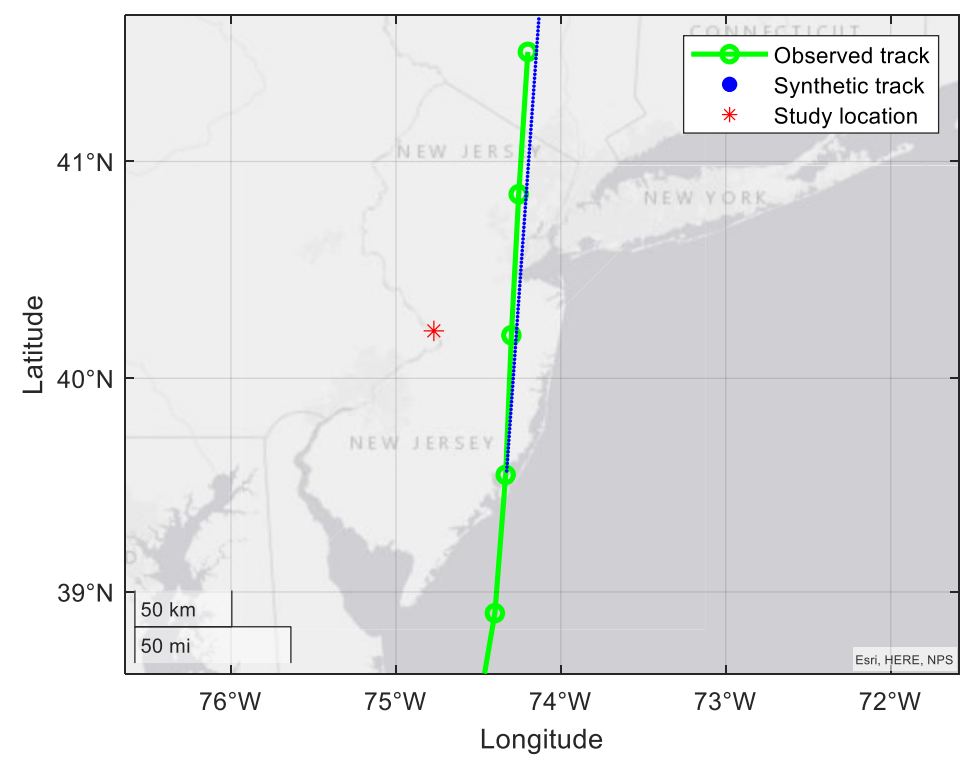

Figure 3-35. Synthetic and observed storm track related to Tropical Storm Fay.

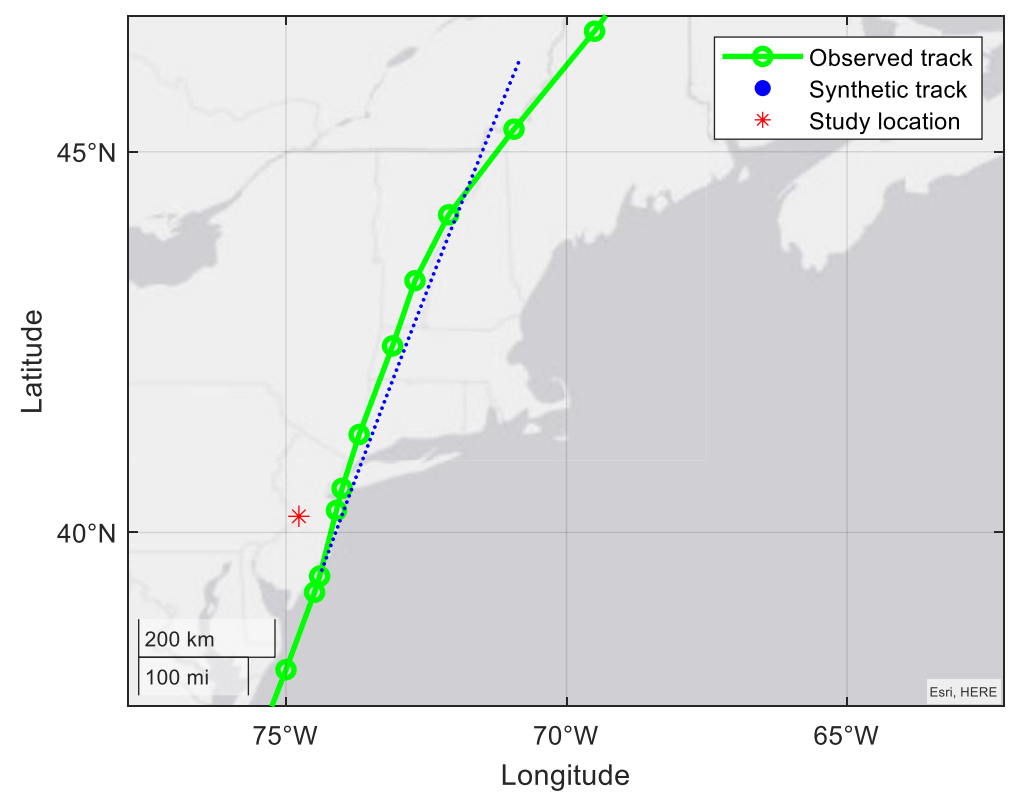

Figure 3-36. Synthetic and observed storm track related to Hurricane Irene. 


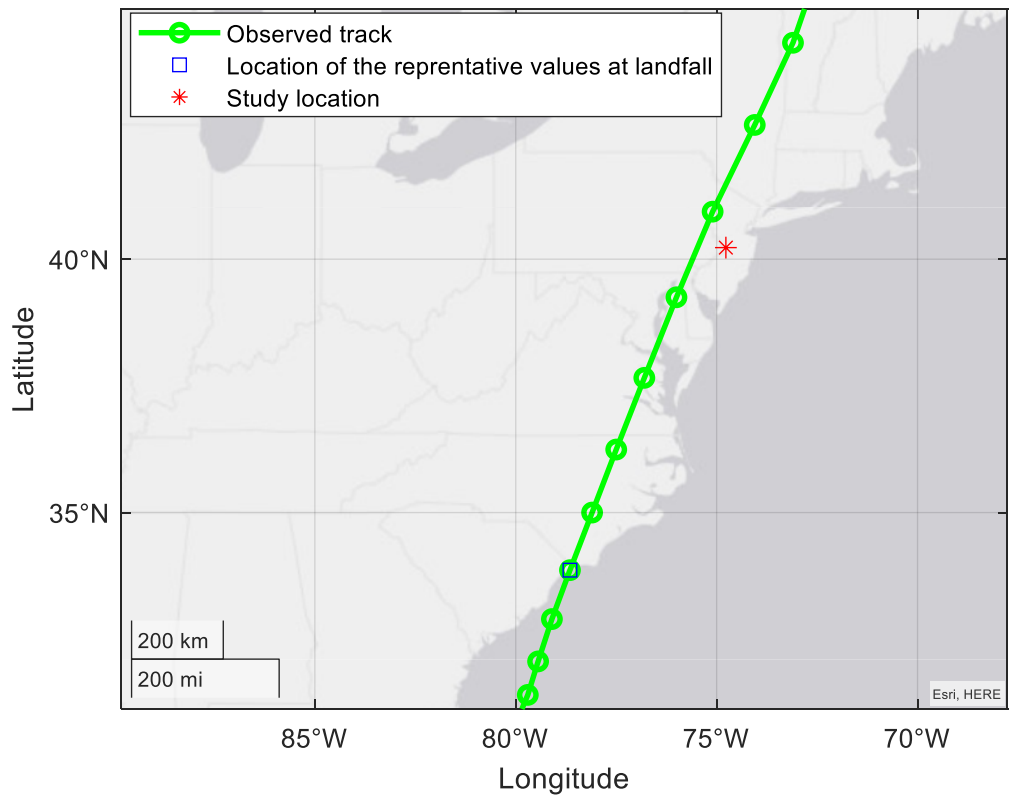

Figure 3-37. Location of the representative point for extracting information at landfall location for Hurricane Isaias.

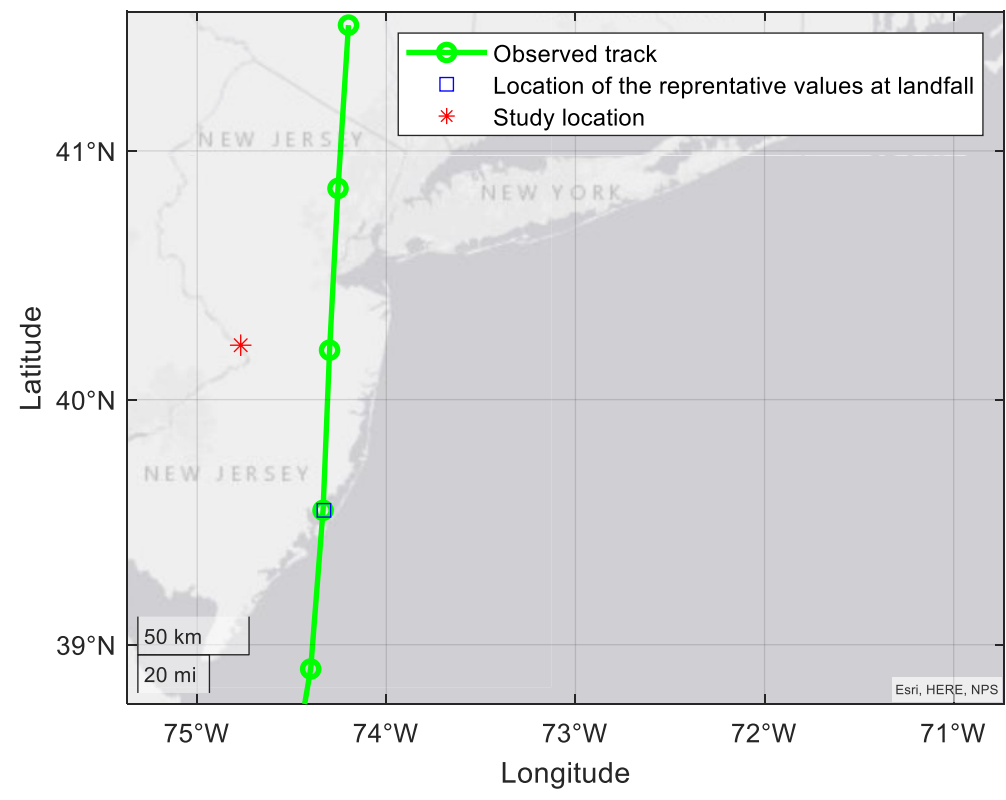

Figure 3-38. Location of the representative point for extracting information at landfall location for Tropical Storm Fay. 


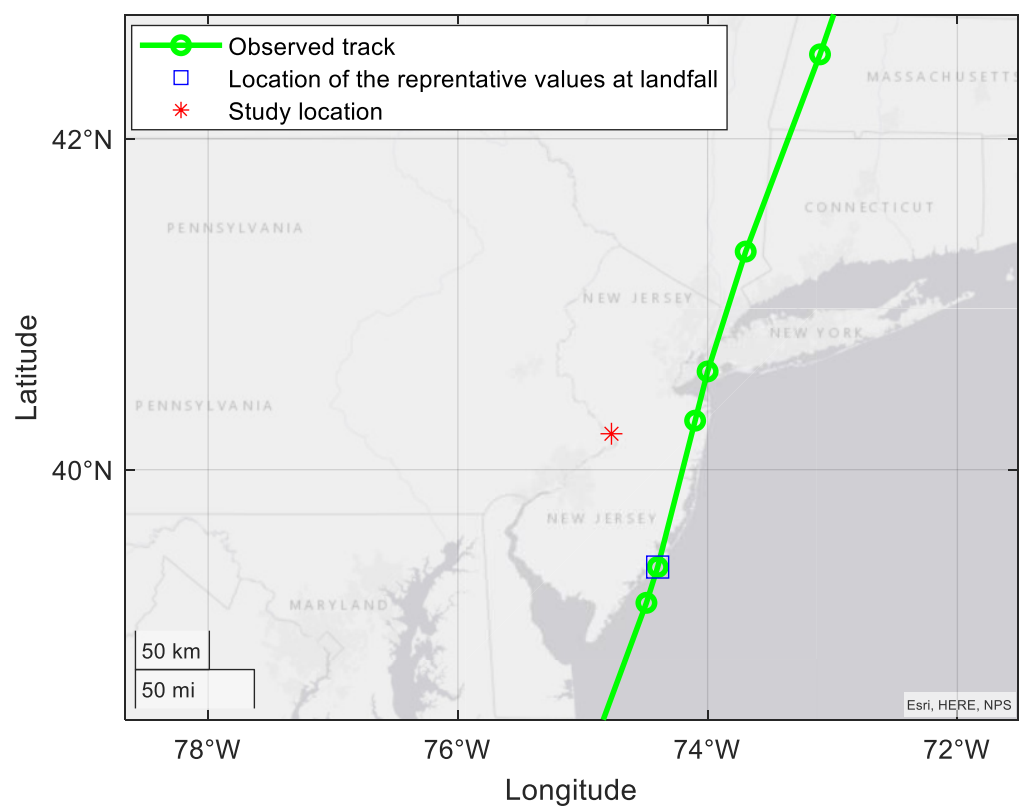

Figure 3-39. Location of the representative point for extracting information at landfall location for Hurricane Irene.

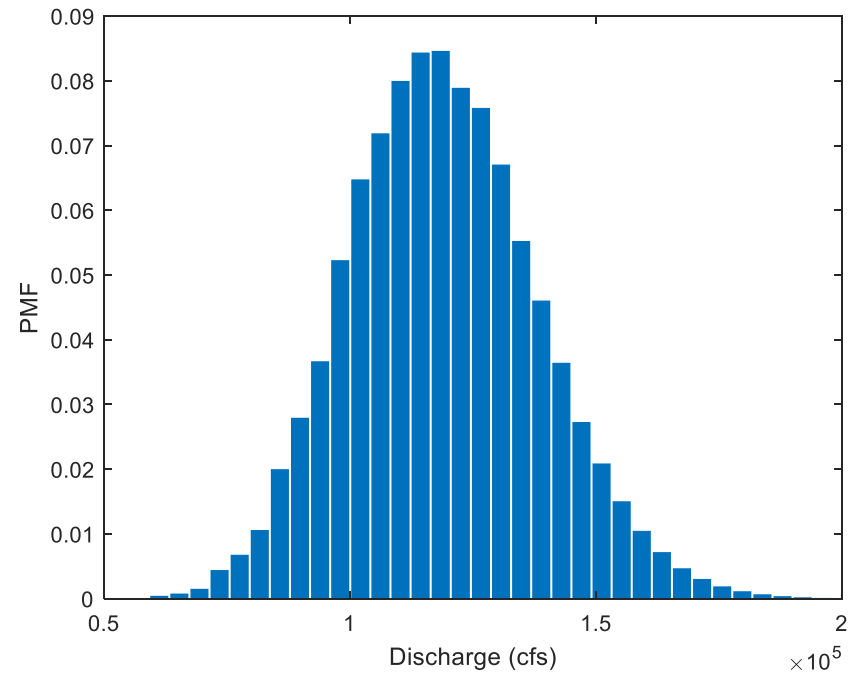

Figure 3-40. Posterior distribution of the modeled discharge values for Hurricane Isaias. 


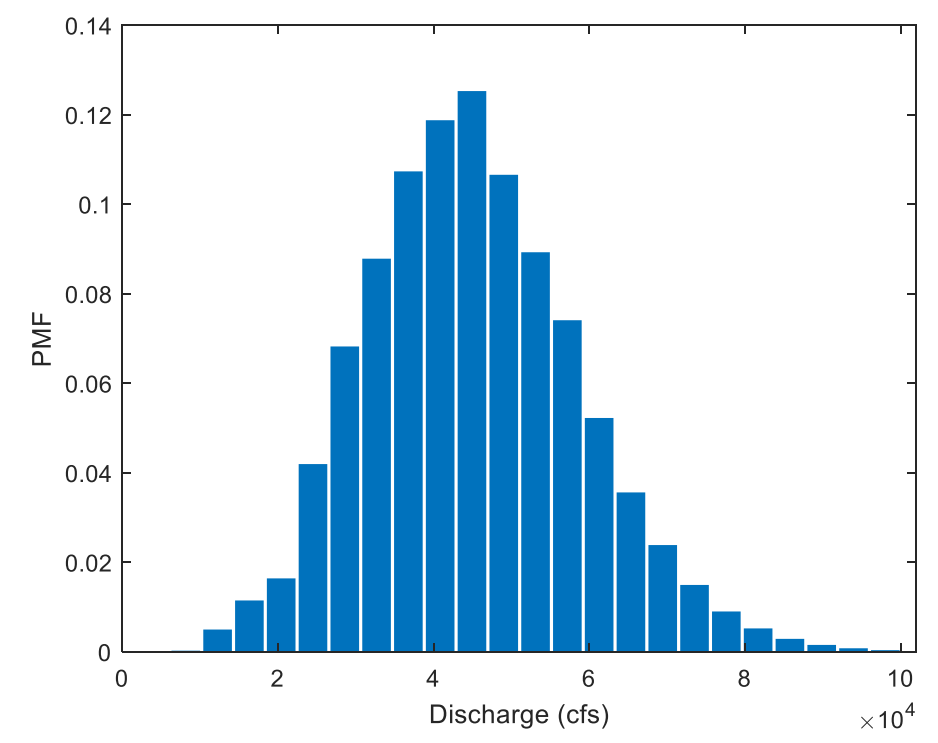

Figure 3-41. Posterior distribution of the modeled discharge values for Tropical Storm Fay.

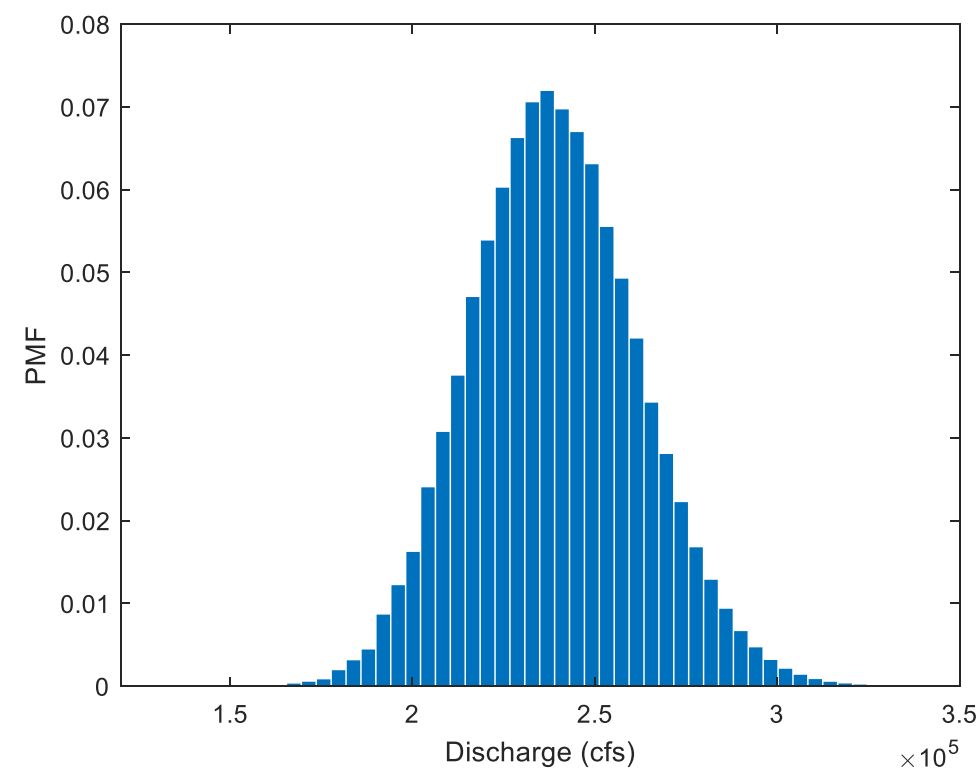

Figure 3-42. Posterior distribution of the modeled discharge values for Hurricane Irene.

\subsection{DISCUSSION}

Based on this coastal case study, strengths and limitations of the Bayesian-motivated MMF assessment approach were identified. The primary advantage of Bayesian-motivated approaches is the capability to (explicitly) incorporate physical process knowledge in the probabilistic assessment. The Bayesianmotivated approach develops a series of conditional probability distributions that represent the physical relationships between quantities, "builds up" the joint distribution from those conditional relationships, and then "integrates over" that joint distribution to derive marginal quantities (and specifically, probabilities of exceedance) of interest. This capability to integrate physical process knowledge is particularly useful in applications with (1) limited data (common in single-mechanism PFHAs and 
exacerbated when considering MMFs) and (2) a need to estimate flood hazards associated with moderate to long return periods (including hazard severities that may not appear within the historical record). However, the Bayesian-motivated approach is not without challenges.

Similar to statistics-based approaches, the Bayesian-motivated approach typically requires statistical analysis of observational or synthetic data series (e.g., storm parameters, tide gage data, river discharge data). This means that many of the statistical modeling challenges articulated in conjunction with the inland case study carry over into the Bayesian approach, including challenges associated with statistical modeling assumptions.

Furthermore, physical process knowledge is necessary to build the probabilistic model. Thus, the probabilistic model is necessarily limited by the state of knowledge. When physical processes are not well understood or associated with epistemic uncertainties, these limitations affect the ability to develop a robust Bayesian-motivated model.

The computational effort associated with the Bayesian-motivated model is non-negligible. Numerical, analytical, empirical, or other models are necessary to describe the relationship between involved quantities. In the case study developed herein, five empirical and surrogate models were used (plus several additional models consisting of linear superposition). Although the development of these empirical and surrogate models was not trivial, the use of more sophisticated numerical models would increase the computational requirements. Furthermore, the use of a Monte Carlo simulation to reduce discretization error in the development of discrete conditional PMFs requires a potentially significant computational effort. Finally, the process of performing calculations necessary to evaluate the expressions shown in Eq. (3.2) can be more challenging than statistical approaches. 



\section{SUMMARY}

This report documents two illustrative case study applications. The first case study focused on inland flooding, specifically snowmelt-driven extreme streamflow events, and used copulas as the main computational approach. The inland case study application demonstrated general procedures involved in the application copulas (e.g., statistical modeling steps). In addition, the case study helped to identify the strengths and limitations of the copula-based MMF assessment approach. A key advantage of the copulabased approach is the general conceptual consistency with conventional univariate PFHA approaches, making the extension from single-mechanism to multi-mechanism assessments straightforward. Copulabased approaches provide flexibility in statistical modeling choices. However, as with univariate approaches, results derived from copula-based approaches are sensitive to statistical modeling assumptions, and limited data may be insufficient to constrain those choices. Furthermore, although bivariate copula analyses are relatively straightforward to implement, extension beyond two variables becomes mathematically more complex.

The second case study considered coastal hazards and used a Bayesian-motivated approach for probabilistic hazard assessment. An illustrative hazard curve was developed for river discharge accounting for the effects of river base flow, hurricane-induced surge, tides, and precipitation-induced runoff. The case study application demonstrates the general conceptual approach to implementing a Bayesian-motivated approach and used a series of empirical and surrogate models to demonstrate implementation. The case study helped to identify the strengths and limitations of the Bayesian-motivated modeling approach. The key advantage of the Bayesian-motivated modeling approach is the capability to incorporate physical process knowledge in the probabilistic assessment. Thus, the approach can serve to overcome certain data limitations and support estimation hazards of associated with moderate to long return periods. However, the Bayesian-motivated approach typically requires statistical analysis of observational or synthetic data series, which means that analyses can be sensitive to statistical modeling assumptions. Furthermore, the Bayesian-motivated approach simply reflects the state of knowledge. Thus, models derived with a Bayesian-motivated approach are subject to existing knowledge gaps. Finally, the computational effort associated with the Bayesian-motivated model is non-negligible and may be substantial. The process of performing calculations necessary to estimate exceedance probabilities of interest can be more challenging than statistical approaches.

Overall, these two case studies provide examples to illustrate two approaches for the probabilistic assessment of MMF hazards. Given the flexibilities of both approaches, we believe that they can be applied more broadly in other MMF applications, not limited to the two specific cases here. Future efforts can be to explore the best practices to incorporate MMF analysis in PFHA, as well as to investigate other suitable methods / tools for more convenient MMF applications. 



\section{REFERENCES}

Addor, N., Newman, A. J., Mizukami, N., and Clark, M. P. (2017). "The CAMELS Data Set: Catchment Attributes and Meteorology for Large-Sample Studies." Hydrol. Earth Syst. Sci., 21, 5293-5313, doi:10.5194/hess-21-5293-2017.

Al Kajbaf, A., and Bensi, M. (2020). "Application of surrogate models in estimation of storm surge:A comparative assessment.” Applied Soft Computing, 91, 106184.

Akaike, H. (1973). "Information theory and an extension of the maximum likelihood principle," 2nd International Symposium on Information Theory, Tsahkadsor, Armenia, September 2-8, 1971, $267-$ 281. Republished in Kotz, S., Johnson, N. L., eds. (1992), Breakthroughs in Statistics, I, SpringerVerlag, 610-624.

Ashfaq, M., Ghosh, S., Kao, S.-C., Bowling, L. C., Mote, P., Touma, D., Rauscher, S. A., and Diffenbaugh, N. S. (2013). "Near-term Acceleration of Hydroclimatic Change in the Western U.S." J. Geophys. Res., 118, 10676-10693, doi:10.1002/jgrd.50816.

Bass, B., and Bedient, P. (2018). "Surrogate modeling of joint flood risk across coastal watersheds." Journal of Hydrology, 558, 159-173.

Bensi, M., Mohammadi, S., Kao, S.-C., and DeNeale, S. T. (2020). "MULTI-MECHANISM FLOOD HAZARD ASSESSMENT: CRITICAL REVIEW OF CURRENT PRACTICE AND APPROACHES." 104.

Bevacqua, E., Maraun, D., Vousdoukas, M. I., Voukouvalas, E., Vrac, M., Mentaschi, L., and Widmann, M. (2019). "Higher probability of compound flooding from precipitation and storm surge in Europe under anthropogenic climate change." Science Advances, 5(9), eaaw5531. doi:10.1126/sciadv.aaw5531.

David, C. H., Maidment, D. R., Niu, G.-Y., Yang, Z.-L., Habets, F., and Eijkhout, V. (2011). "River network routing on the NHDPlus dataset.” J. Hydrometeorol., 12(5), 913-934. doi:10.1175/2011JHM1345.1.

Genest, C., and Rivest, L.-P. (1993). "Statistical inference procedures for bivariate Archimedean copulas." Journal of the American Statistical Association, 88(423), 1034-1043. doi:10.2307/2290796.

Genest, C., Favre, A.-C., Béliveau, J., and Jacques, C. (2007). "Metaelliptical Copulas and Their Use in Frequency Analysis of Multivariate Hydrological Data." Water Resour. Res., 43, W09401, doi:10.1029/2006WR005275.

"IBTrACS - International Best Track Archive for Climate Steetchip." (n.d.). <https://www.ncdc.noaa.gov/ibtracs/index.php?name=ibtracs-data > (Apr. 28, 2021).

Jia, G., and Taflanidis, A. A. (2013). "Kriging metamodeling for approximation of high-dimensional wave and surge responses in real-time storm/hurricane risk assessment." Computer Methods in Applied Mechanics and Engineering, 261-262, 24-38.

Jia, G., Taflanidis, A. A., Nadal-Caraballo, N. C., Melby, J. A., Kennedy, A. B., and Smith, J. M. (2016). "Surrogate modeling for peak or time-dependent storm surge prediction over an extended coastal region using an existing database of synthetic storms." Natural Hazards, 81(2), 909-938.

Kaplan, J., and DeMaria, M. (1995). "A Simple Empirical Model for Predicting the Decay of Tropical Cyclone Winds after Landfall.” Journal of Applied Meteorology (1988-2005), American Meteorological Society, 34(11), 2499-2512. 
Kaplan, J., and Demaria, M. (2001). "On the Decay of Tropical Cyclone Winds after Landfall in the New England Area." Journal of Applied Meteorology (1988-2005), American Meteorological Society, 40(2), 280-286.

Kao, S.-C. and Govindaraju, R. S. (2007). "A Bivariate Frequency Analysis of Extreme Rainfall with Implications for Design.” J. Geophys. Res.-Atmos., 112, D13119, doi:10.1029/2007JD008522.

Kew, S. F., Selten, F. M., Lenderink, G., and Hazeleger, W. (2013). "The simultaneous occurrence of surge and discharge extremes for the Rhine delta." Natural Hazards and Earth System Sciences, 13(8), 2017-2029. doi:10.5194/nhess-13-2017-2013.

Marks, F., Kappler, G., DeMaria, M., and Collins, F. (2002). "DEVELOPMENT OF A TROPICAL CYCLONE RAINFALL CLIMATOLOGY AND PERSISTENCE (R-CLIPER) MODEL.” 3.

Nadal-Caraballo, N. C., Melby, J. A., Gonzalez, V. M., and Cox, A. T. (2015). "Coastal Storm Hazards from Virginia to Maine.” 221.

Naz, B. S., Kao, S.-C., Ashfaq, M., Rastogi, D., Mei, R., and Bowling, L. C. (2016). "Regional Hydrologic Response to Climate Change in the Conterminous United States Using High-resolution Hydroclimate Simulations." Global Planet. Change, 143, 100-117, doi:10.1016/j.gloplacha.2016.06.003.

Nelsen, R. B. (2006). An Introduction to Copulas, Springer-Verlag New York, Inc., New York, NY.

Newman, A. J., Sampson, K., Clark, M. P., Bock, A., Viger, R. J., and Blodgett, D. (2014). A LargeSample Watershed-Scale Hydrometeorological Dataset for the Contiguous USA, UCAR/NCAR, Boulder, CO, doi:10.5065/D6MW2F4D.

NOAA. (2018). "HURDAT Re-analysis." < https://www.aoml.noaa.gov/hrd/hurdat/Data_Storm.html $>$ (Apr. 23, 2021).

NOAA. (2021). " TRENTON MARINE TERMINAL, NJ - Station ID: 8539993." <https://tidesandcurrents.noaa.gov/stationhome.html?id=8539993> (Sep. 13, 2021).

NRC. (2018). "Strategic Plan: Fiscal Years 2018-2022 (NUREG-1614, Volume 7)." <https://www.nrc.gov/reading-rm/doc-collections/nuregs/staff/sr1614/v7/index.html> (Sep. 13, 2021).

NRC. (2014). "Probabilistic Flood Hazard Assessment Research Plan." <https://www.nrc.gov/docs/ML1429/ML14296A442.pdf> (Sep. 13, 2021).

Oubeidillah, A. A., Kao, S.-C., Ashfaq, M., Naz, B. S., and Tootle, G. (2014). "A Large-Scale, HighResolution Hydrological Model Parameter Data Set for Climate Change Impact Assessment for the Conterminous US.” Hydrol. Earth Syst. Sci., 18, 67-84, doi:10.5194/hess-18-67-2014.

Oubeidillah, A. A., Kao, S.-C., Ashfaq, M., Naz, B., and Tootle, G. (2013). “A large-scale, highresolution hydrological model parameter dataset for climate change impact assessment for the conterminous United States." Hydrology and Earth System Sciences Discussions, 10, 9575-9613.

Rao, A. R., and Hamed, K. H. (2000). Flood Frequency Analysis, CRC Press LLC, FL.

Saunders, R., and Laud, P. (1980). "The multidimensional Kolmogorov goodness-of-fit test," Biometrika, 67(1), 237. doi:10.1093/biomet/67.1.237.

Schwarz, G. E. (1978). "Estimating the dimension of a model." Annals of Statistics, 6(2): 461-464, doi:10.1214/aos/1176344136.

Thornton, P. E., Running, S. W., and White, M. A. (1997). "Generating Surfaces of Daily Meteorology Variables over Large Regions of Complex Terrain.” J. Hydrol., 190, 214-251, doi:10.1016/S00221694(96)03128-9. 
Toro, G. R., Resio, D. T., Divoky, D., Niedoroda, A. Wm., and Reed, C. (2010). "Efficient jointprobability methods for hurricane surge frequency analysis." Ocean Engineering, A Forensic Analysis of Hurricane Katrina's Impact: Methods and Findings, 37(1), 125-134.

Tuleya, R. E., DeMaria, M., and Kuligowski, R. J. (2007). "Evaluation of GFDL and Simple Statistical Model Rainfall Forecasts for U.S. Landfalling Tropical Storms." Weather and Forecasting, American Meteorological Society, 22(1), 56-70.

USACE. (2021). "Coastal Hazards System, V2.0." < https://chs.erdc.dren.mil/> (Sep. 13, 2021).

USGS. (2021). " USGS 01463500 Delaware River at Trenton NJ." National Water Information System: Web Interface.

<https://waterdata.usgs.gov/nj/nwis/uv/?site_no=01463500\&PARAmeter_cd=00065,00060> (Sep. 13, 2021).

Vernieuwe, H., Vandenberghe, S., De Baets, B., and Verhoest, N. E. C. (2015). "A Continuous Rainfall Model Based on Vine Copulas." Hydrol. Earth Syst. Sci., 19, 2685-2699, doi:10.5194/hess-19-26852015. 


